\title{
Quantum corrections for D-brane models with broken supersymmetry
}

\author{
Wilfried Buchmuller, ${ }^{a}$ Emilian Dudas $^{b}$ and Yoshiyuki Tatsuta ${ }^{a}$ \\ ${ }^{a}$ Deutsches Elektronen-Synchrotron DESY, \\ Notkestraße 85, Hamburg 2260\%, Germany \\ ${ }^{b}$ Centre de Physique Théorique, CNRS, Institut Polytechnique de Paris, \\ Palaiseau 91128, France \\ E-mail: wilfried.buchmueller@desy.de, \\ emilian.dudas@cpht.polytechnique.fr, yoshiyuki.tatsuta@desy.de
}

ABSTRACT: Intersecting D-brane models and their T-dual magnetic compactifications yield attractive models of particle physics where magnetic flux plays a twofold role, being the source of fermion chirality as well as supersymmetry breaking. A potential problem of these models is the appearance of tachyons which can only be avoided in certain regions of moduli space and in the presence of Wilson lines. We study the effective four-dimensional field theory for an orientifold compactification of type IIA string theory and the corresponding toroidal compactification of type I string theory. After determining the Kaluza-Klein and Landau-level towers of massive states in different sectors of the model, we evaluate their contributions to the one-loop effective potential, summing over all massive states, and we relate the result to the corresponding string partition functions. We find that the Wilsonline effective potential has only saddle points, and the theory is therefore driven to the tachyonic regime. There tachyon condensation takes place and chiral fermions acquire a mass of the order of the compactification scale. We also find evidence for a tachyonic behaviour of the volume moduli. More work on tachyon condensation is needed to clarify the connection between supersymmetry breaking, a chiral fermion spectrum and vacuum stability.

Keywords: D-branes, Field Theories in Higher Dimensions, Flux compactifications

ARXIV EPRINT: 1909.03007 


\section{Contents}

1 Introduction 1

2 Intersecting D-brane model 3

3 T-dual toroidal flux compactification $\quad 8$

4 Matter sector $\quad 14$

4.1 Antisymmetric tensor ( $a a^{\prime}$-sector) 14

4.2 Chiral matter ( $a b$-sector) 20

5 Higgs sector $\quad 24$

$5.1 b c$-sector 28

6 Effective potential $\quad 28$

6.1 Field theory calculation 28

6.2 String calculation 32

6.3 Volume-moduli potential 35

7 Tachyon condensation $\quad 35$

$7.1 b c^{\prime}$-sector 37

$\begin{array}{lll}7.2 & b c \text {-sector } & 37\end{array}$

8 Conclusions and open questions $\quad 38$

A Embedding $\mathrm{U}(N)$ into $\mathrm{SO}(2 N) \quad 39$

B Commutators $\quad 42$

$\begin{array}{ll}\text { C Superfield components } & 43\end{array}$

D Jacobi functions $\quad 44$

\section{Introduction}

Intersecting D-brane models and their T-dual magnetic compactifications provide attractive and intuitive string theory compactifications to four dimensions with chiral fermion spectra [1,2]. The main emphasis in model building has been on the construction of vacua with unbroken $\mathcal{N}=1$ supersymmetry (for a review and references, see [3, 4]), but in absence of any hint for supersymmetry at the Large Hadron Collider, models where supersymmetry is broken at a high scale, in the spirit of 'split supersymmetry' $[5,6]$ or 'split symmetries' [7, 8], are also of current interest. 
An intriguing aspect of magnetic compactifications is the connection between fermion chirality and supersymmetry breaking [9], which occurs in compactifications of type I strings on tori and orbifolds [10-12] and in the related intersecting D-brane models [11, 13, 14]. This setup allows to construct models which come very close to the Standard Model of particle physics [15-18]. Generically, magnetic compactifications have tachyonic instabilities of Nielsen-Olesen type [19]. Originally, one could hope to relate such an instability to electroweak symmetry breaking $[9,15,16]$ in case of a low string scale and large extra dimensions. This is no longer viable but the structure of the setup is rich enough to incorporate in principle also split supersymmetry [20,21].

The goal of this paper is the computation of quantum corrections for string compactifications with magnetic background flux. This is partly motivated by the recent observation that in quantum corrections to Wilson-line scalars large cancellations occur [22-25] due to the presence of magnetic flux. This suggests that in appropriate compactifications similar cancellations may occur in quantum corrections to Higgs masses, which would be important in view of the hierarchy problem. In order to address these questions we extend the previous calculations for six-dimensional field theory models to a full string compactification on magnetized tori. Notice, that another motivation of our effective field theory approach is that, whenever supersymmetry is broken by magnetic fluxes, in string theory NSNS tadpoles are generated that make any quantum computation very hard, both conceptually and technically (see, for example, [26]).

Our starting point is the construction of an intersecting brane model with broken supersymmetry in a matter sector without tachyons and with chiral fermions which can acquire mass via the Higgs mechanism. For simplicity, and to facilitate the computation of quantum corrections, we choose as unbroken gauge group $\mathrm{U}(N) \times \mathrm{U}(1) \times \mathrm{U}(1)$ rather than the Standard Model gauge group. The model has a Higgs sector and antisymmetric tensor fields with fermions in vector-like representations. Some scalar masses in these sectors depend on the distance between branes that are parallel in some tori. These moduli correspond to Wilson-line scalars in the T-dual picture. They become tachyonic if the branes come close to each other. At tree level the Wilson-line potential is flat. However, as we shall see, one-loop quantum corrections make it concave, implying that the system is driven into the tachyonic regime of moduli space.

After determining intersection numbers and scalar masses for the D-brane model, we turn to the T-dual magnetic compactification which is better suited to evaluate the fourdimensional (4d) effective field theory. Starting from the 10d SO(32) Super-Yang-Mills Lagrangian expressed in terms of $\mathcal{N}=1$ vector and chiral superfields [27, 28], we compute the $4 \mathrm{~d}$ effective action for a toroidal compactification with three $\mathrm{U}(1)$ magnetic background fluxes that break $\mathrm{SO}(32)$ to $\mathrm{U}(N) \times \mathrm{U}(1) \times \mathrm{U}(1)$. For each sector of the model we determine the Kaluza-Klein (KK) and Landau-level (LL) towers of mass eigenstates of vectors, fermions and scalars. The calculations are based on the harmonic oscillator algebra of covariant derivatives in a flux background [9, 24, 29-31]. The mass spectra are compared with the string formula of Bachas, also in view of supersymmetries that remain unbroken for particular choices of magnetic fluxes in some sectors. 
In the Higgs sector branes are parallel in some tori and, knowing the spectrum of massive KK and LL states, we compute the effective potential as function of magnetic flux and Wilson lines. The effective potential is also obtained in the field theory limit of the corresponding string partition function, and the two results agree. As function of the Wilson line the potential is concave and there are no local minima. Hence, the tree level vacua with non-vanishing Wilson lines are unstable. This is a new result of our paper. For vanishing Wilson lines tachyon condensation takes place and all chiral fermions acquire masses of the order of the compactification scale.

The contributions to the effective potential from the various sectors are most easily obtained from the corresponding string partition functions. In sectors without Wilson lines we also calculate the effective potential as function of the volume moduli of the three tori. We find evidence that also in this case the system is driven to the tachyonic regime of moduli space, which would imply that the only vacuum state corresponds to the decompactification limit. A further, well-known problem is the NSNS tadpole (see, for example, [26]) in case of broken supersymmetry.

The paper is organized as follows. The intersecting D-brane model and its T-dual magnetic compactification are discussed in sections 2 and 3, respectively. Mass eigenstates and mass spectra are derived in sections 4 and 5, and the effective one-loop potential is computed in section 6 . Section 7 deals with tachyon condensation. The appendices A and B give details concerning the embedding of the various sectors of the model in the adjoint representation of $\mathrm{SO}(32)$, and in the appendices $\mathrm{C}$ and $\mathrm{D}$ some formulae are collected for superfield components and Jacobi functions, respectively.

\section{Intersecting D-brane model}

We are interested in a D-brane model with broken supersymmetry, which contains a 'matter sector' with chiral fermions and a 'Higgs sector' with vector-like fermions such that vacuum expectation values of Higgs fields can give mass to the chiral fermions. As a simple example, we choose the gauge group

$$
G=\mathrm{U}(N) \times \mathrm{U}(1) \times \mathrm{U}(1),
$$

corresponding to a stack of $N$ branes, $a$, and two single branes, $b$ and $c$. The fermions are supposed to be chiral with respect to $\mathrm{U}(1) \times \mathrm{U}(1)$ and vector-like with respect to the 'colour group' $\mathrm{U}(N)$. Following $[11,16]$, we start from type IIA string theory compactified on a rectangular factorized torus $T^{6}=T_{1}^{2} \times T_{2}^{2} \times T_{3}^{2}$ with real coordinates $x_{4}, \ldots, x_{9}$ and complex coordinates $z_{i}=\left(x_{2+2 i}+i x_{3+2 i}\right) / 2, i=1,2,3$, with the identifications $z_{i} \sim z_{i}+L_{i} / 2$, $z_{i} \sim z_{i}+i L_{i}^{\prime} / 2$. An orientifold is obtained by dividing out the discrete symmetry $\Omega \mathcal{R}(-1)^{F_{L}}$, where $\Omega$ is worldsheet parity, $F_{L}$ is left-moving fermion number, and $\mathcal{R}$ is a reflection symmetry of $T^{6}$,

$$
\mathcal{R}:\left(z_{1}, z_{2}, z_{3}\right) \rightarrow\left(\bar{z}_{1}, \bar{z}_{2}, \bar{z}_{3}\right) .
$$

The orientifold has eight $O 6$-planes along Minkowski space and the directions $x_{3+2 i}$ that are invariant under $\mathcal{R}$. The orientifold planes are localized at the fixed points $\left(\hat{z}_{1}, \hat{z}_{2}, \hat{z}_{3}\right)$, $\hat{z}_{i}=\left(0, i L^{\prime} / 4\right)$. Each orientifold plane has RR charge $Q_{O 6}=-2$ in units of a D6-brane 


\begin{tabular}{|l|ccc|}
\hline Branes, gauge groups & $\left(n^{1}, m^{1}\right)$ & $\left(n^{2}, m^{2}\right)$ & $\left(n^{3}, m^{3}\right)$ \\
\hline$a, \mathrm{U}(N)$ & $(1,0)$ & $(1,2)$ & $(1,1)$ \\
$b, \mathrm{U}(1)$ & $(1,1)$ & $(1, l)$ & $(1,-2)$ \\
$c, \mathrm{U}(1)$ & $(1,1)$ & $(1,-l)$ & $(1,2)$ \\
\hline
\end{tabular}

Table 1. Intersecting D-brane model. Wrapping numbers of a stack of $N$ branes, $a$, and two single branes, $b$ and $c$.

charge. Cancellation of the total RR charge requires 16 D6-branes together with 16 mirror D6 branes to satisfy the reflection symmetry $\mathcal{R}$ of the compact space. A brane $e$ is wrapped around the 1-cycles $\left[a_{i}\right]$ and $\left[b_{i}\right]$ of the 2 -tori $T_{i}^{2}$ with wrapping numbers $n_{e}^{i}$ and $m_{e}^{i}$, yielding for the wrapped 3 -cycle of the brane the homology class $^{1}$

$$
\left[\Pi_{e}\right]=\otimes_{i}\left(n_{e}^{i}\left[a_{i}\right]+m_{e}^{i}\left[b_{i}\right]\right) .
$$

The homology class $\left[\Pi_{e^{\prime}}\right]$ of the mirror brane is obtained from $\left[\Pi_{e}\right]$ by replacing $m_{e}^{i}$ by $-m_{e}^{i}$. In case of stacks of $N_{e}$ branes, leading to gauge symmetries $\mathrm{U}\left(N_{e}\right)$, the RR tadpole cancellation condition can now be written as

$$
\sum_{e} N_{e}\left[\Pi_{e}\right]-2\left[\Pi_{O 6}\right]=0
$$

where $\left[\Pi_{O 6}\right]=8 \otimes_{i}\left[a_{i}\right]$ is the homology class of the orientifold plane.

We are interested in the gauge group $\mathrm{U}(N) \times \mathrm{U}(1) \times \mathrm{U}(1)$, corresponding to one stack of $N$ branes, $a$, with gauge group $\mathrm{U}(N)$, and two further single $\mathrm{U}(1)$ branes, $b$ and $c$. Table 1 shows a set of wrapping numbers which can be consistent with the wanted gauge group $\mathrm{U}(N) \times \mathrm{U}(1) \times \mathrm{U}(1)$. We have chosen all wrapping number in the $x_{3+2 i}$ directions equal, $n^{i}=1$, and one wrapping number in the first torus as zero, $m_{a}^{1}=0$. In this case, the tadpole conditions (2.4) read explicitly,

$$
\begin{aligned}
N+2 & =16, \\
N m_{a}^{2} m_{a}^{3}+m_{b}^{2} m_{b}^{3}+m_{c}^{2} m_{c}^{3} & =0 \\
m_{b}^{1} m_{b}^{3}+m_{c}^{1} m_{c}^{3} & =0 \\
m_{b}^{1} m_{b}^{2}+m_{c}^{1} m_{c}^{2} & =0 .
\end{aligned}
$$

One easily verifies that these equations are solved by the ansatz in table 1 , with $N=14$, $l=7$. The chosen wrapping numbers imply that not all branes intersect in all tori: $a$ and $a^{\prime}$, and $b$ and $c$ are parallel in the first torus, whereas $b$ and $c^{\prime}$ are parallel in the second and in the third torus. This situation is illustrated in figure 1.

On each brane an $\mathcal{N}=4$ supermultiplet of zero-modes in the adjoint representation of the gauge group is localized. The branes intersect at angles determined by the wrapping numbers. At these intersections fermions and scalars in bi-fundamental representations $\left(N_{e}, \bar{N}_{f}\right)$ are localized. For non-zero intersection numbers

$$
I_{e f}=\otimes_{i}\left(n_{e}^{i} m_{f}^{i}-m_{e}^{i} n_{f}^{i}\right)
$$

\footnotetext{
${ }^{1}$ We mostly follow the conventions of [4].
} 

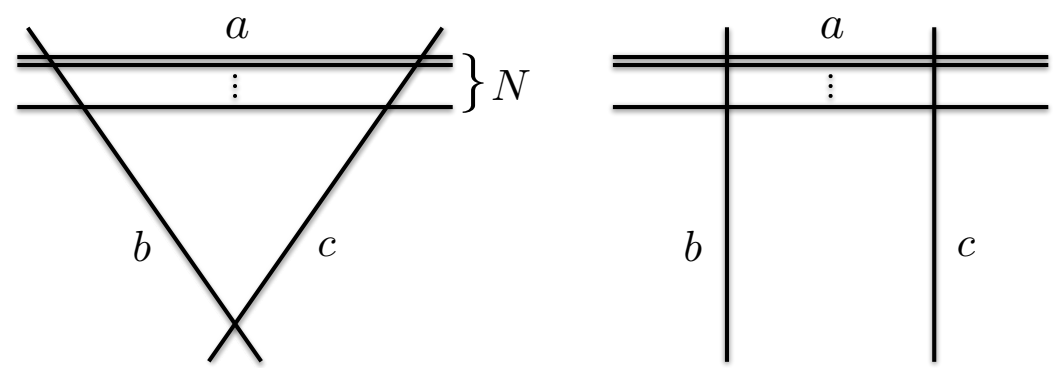

Figure 1. Left: intersections of brane stack $a$ with branes $b$ and $c$, and brane $b$ with $c$ in the second torus $T_{2}^{2}$; right: intersections of brane stack $a$ with branes $b$ and $c$ in torus $T_{1}^{2}$ where branes $b$ and $c$ are parallel.

\begin{tabular}{|c|cc|}
\hline Brane sector & Intersection number $I$ & $4 \mathrm{~d}$ fermions $(\mathrm{L})$ \\
\hline$a b+b a$ & $-3(l-2)$ & $\bar{N}_{1,0}$ \\
$a c+c a$ & $-(l+2)$ & $\bar{N}_{0,1}$ \\
$a b^{\prime}+b^{\prime} a$ & $l+2$ & $N_{1,0}$ \\
$a c^{\prime}+c^{\prime} a$ & $3(l-2)$ & $N_{0,1}$ \\
$a a^{\prime}$ & 0 & $N(N-1) / 2, \bar{N}(\bar{N}-1) / 2$ \\
$b c+c b$ & 0 & $1_{1,-1}, 1_{-1,1}$ \\
$b c^{\prime}+c^{\prime} b$ & 0 & $1_{1,1}, 1_{-1,-1}$ \\
\hline
\end{tabular}

Table 2. Chiral and vector-like representations of left-handed fermions at various brane intersections.

the fermion spectrum is chiral. The fermions are left-handed for $I_{e f}>0$ and right-handed for $I_{e f}<0$, corresponding to left-handed fermions in the complex conjugate representation $\left(\bar{N}_{e}, N_{f}\right)$. At the intersections of the brane system defined in table 1 one obtains the lefthanded fermions listed in table 2. There are matter fields that carry 'colour', transforming as $N$ or $\bar{N}$ under $\mathrm{SU}(N)$. They form a chiral representation of the full gauge group, whereas colour singlet 'Higgs fields' form vector-like representations. The quantum numbers of the chiral fermions allow Yukawa couplings that are most conveniently expressed in terms of the associated chiral superfields,

$$
\mathcal{L}_{Y} \supset \sum_{r, s}^{3(l-2)} y_{r s}^{(1)} \overline{\boldsymbol{N}}_{1,0}^{r} \boldsymbol{N}_{0,1}^{s} \mathbf{1}_{-1,-1}+\sum_{r, s}^{l+2} y_{r s}^{(2)} \overline{\mathbf{N}}_{0,1}^{r} \mathbf{N}_{1,0}^{s} \mathbf{1}_{-1,-1} .
$$

These couplings lead to fermion mass terms after a vacuum expectation value $\left\langle 1_{-1,-1}\right\rangle \neq 0$ breaks the chiral group $\mathrm{U}(1) \times \mathrm{U}(1)$ to the diagonal $\mathrm{U}(1)$ subgroup. The complete list of Yukawa couplings will be given in the subsequent section.

In the brane sector $a a^{\prime}, b b^{\prime}$ and $c c^{\prime}$ chiral fermions in symmetric and antisymmetric representations of the gauge group occur with multiplicities

$$
\begin{aligned}
n_{\mathrm{sym}, e} & =\frac{1}{2}\left(I_{e e^{\prime}}-I_{e, O 6}\right)=-4 m_{e}^{1} m_{e}^{2} m_{e}^{3}\left(n_{e}^{1} n_{e}^{2} n_{e}^{3}-1\right), \\
n_{\text {asym }, e} & =\frac{1}{2}\left(I_{e e^{\prime}}+I_{e, O 6}\right)=-4 m_{e}^{1} m_{e}^{2} m_{e}^{3}\left(n_{e}^{1} n_{e}^{2} n_{e}^{3}+1\right), e=a, b, c .
\end{aligned}
$$




\begin{tabular}{|c|ccc|}
\hline Brane sectors & $\left.4 \pi \alpha^{\prime} M_{1}^{2}\right|_{e f}$ & $\left.4 \pi \alpha^{\prime} M_{2}^{2}\right|_{e f}$ & $\left.4 \pi \alpha^{\prime} M_{3}^{2}\right|_{e f}$ \\
\hline$a b, a c^{\prime}$ & $-\rho_{1}+(l-2) \rho_{2}+3 \rho_{3}$ & $\rho_{1}-(l-2) \rho_{2}+3 \rho_{3}$ & $\rho_{1}+(l-2) \rho_{2}-3 \rho_{3}$ \\
$a b^{\prime}, a c$ & $-\rho_{1}+(l+2) \rho_{2}+\rho_{3}$ & $\rho_{1}-(l+2) \rho_{2}+\rho_{3}$ & $\rho_{1}+(l+2) \rho_{2}-\rho_{3}$ \\
$a a^{\prime}$ & $4 \rho_{2}+2 \rho_{3}$ & $-4 \rho_{2}+2 \rho_{3}$ & $4 \rho_{2}-2 \rho_{3}$ \\
$b c$ & $2 l \rho_{2}+4 \rho_{3}$ & $-2 l \rho_{2}+4 \rho_{3}$ & $2 l \rho_{2}-4 \rho_{3}$ \\
$b c^{\prime}$ & $-2 \rho_{1}$ & $2 \rho_{1}$ & $2 \rho_{1}$ \\
\hline
\end{tabular}

Table 3. Masses of scalars at various brane intersections.

Since in our model $m_{a}^{1}=0$ and $n_{e}^{1}=n_{e}^{2}=n_{e}^{3}=1$ for $e=a, b, c$, there are no chiral fermions in symmetric or antisymmetric representations. As we shall see in the following section, a vector-like pair of fermions in the antisymmetric representation of $\mathrm{SU}(N)$ occurs in the $a a^{\prime}$-sector. The $b b^{\prime}$ - and the $c c^{\prime}$-sector correspond to $\mathrm{U}(1)$ symmetries where such representations are absent.

The masses of bi-fundamental scalars depend on the angles at which the branes intersect. We restrict ourselves to small angles with respect to the orientifold planes,

$$
\tan \theta_{e}^{i}=m_{e}^{i} \rho_{i} \simeq \theta_{e}^{i}, \quad \rho_{i}=\frac{L_{i}^{\prime}}{L_{i}}, \quad i=1,2,3, \quad e=a, b, c,
$$

where $L_{i} /(2 \pi)$ and $L_{i}^{\prime} /(2 \pi)$ are the two radii of the torus $T_{i}^{2}$, respectively. In the T-dual picture small angles correspond to large areas of the dual tori so that we shall be able to use a field theory approximation to string partition functions.

At the intersection of two stacks of branes, $e$ and $f$, one then has three light bifundamental scalars with masses [14]

$$
\begin{aligned}
& \left.4 \pi \alpha^{\prime} M_{1}^{2}\right|_{e f}=-\left|\theta_{e f}^{1}\right|+\left|\theta_{e f}^{2}\right|+\left|\theta_{e f}^{3}\right| \\
& \left.4 \pi \alpha^{\prime} M_{2}^{2}\right|_{e f}=\left|\theta_{e f}^{1}\right|-\left|\theta_{e f}^{2}\right|+\left|\theta_{e f}^{3}\right| \\
& \left.4 \pi \alpha^{\prime} M_{3}^{2}\right|_{e f}=\left|\theta_{e f}^{1}\right|+\left|\theta_{e f}^{2}\right|-\left|\theta_{e f}^{3}\right|,
\end{aligned}
$$

where $\theta_{\text {ef }}^{i}=\theta_{e}^{i}-\theta_{f}^{i}$, with $-\pi / 2 \leq \theta_{\text {ef }}^{i} \leq \pi / 2$. For the model defined in table 1 one obtains

$$
\begin{array}{lll}
\theta_{a}^{1}=0, & \theta_{a}^{2}=2 \rho_{2}, & \theta_{a}^{3}=\rho_{3}, \\
\theta_{b}^{1}=\rho_{1}, & \theta_{b}^{2}=l \rho_{2}, & \theta_{b}^{3}=-2 \rho_{3}, \\
\theta_{c}^{1}=\rho_{1}, & \theta_{c}^{2}=-l \rho_{2}, & \theta_{c}^{3}=2 \rho_{3} .
\end{array}
$$

Using eq. (2.10) these angles yield the scalar mass spectrum at the various brane intersections which is listed in table 3.

In any sector of states stretched between any two stacks of branes $e, f$, some supersymmetry is preserved provided that angles fulfill the following conditions [13],

$$
\begin{aligned}
\theta_{\text {ef }}^{1} \pm \theta_{\text {ef }}^{2} \pm \theta_{\text {ef }}^{3} \neq 0: & \mathcal{N}=4 \rightarrow \mathcal{N}=0, \\
\theta_{\text {ef }}^{1} \pm \theta_{\text {ef }}^{2} \pm \theta_{\text {ef }}^{3}=0: & \mathcal{N}=4 \rightarrow \mathcal{N}=1, \\
\theta_{\text {ef }}^{1} \pm \theta_{\text {ef }}^{2}=0, \theta_{\text {ef }}^{3}=0: & \mathcal{N}=4 \rightarrow \mathcal{N}=2 .
\end{aligned}
$$


In the considered model, a tachyon occurs in the $b c^{\prime}$-sector,

$$
\left.m_{1}^{2}\right|_{b c^{\prime}} \propto-2 \rho_{1}<0 .
$$

A further tachyon appears either in the $a a^{\prime}$-sector or in the $b c$-sector. In both sectors the flux in the first torus is zero, i.e. $\theta^{1}=0$. Choosing $\theta^{2}=\theta^{3}$ yields two massless scalars, avoiding tachyons. For the $a a^{\prime}$-sector this means

$$
\rho_{3}=2 \rho_{2}
$$

which avoids coloured tachyons but implies a second tachyon in the $b c$-sector,

$$
\left.m_{1}^{2}\right|_{b c} \propto(-2 l+4) \rho_{2}<0 .
$$

Together with the two fermionic zero-modes the $a a^{\prime}$-sector then forms a subsystem with $\mathcal{N}=2$ supersymmetry. For the choice $l \rho_{2}=2 \rho_{3}$ the roles the $a a^{\prime}$-sector and the $b c$-sector are reversed.

The condition for absence of tachyons in the $a b$ - and $a c^{\prime}$-sector reads

$$
(8-l) \rho_{2}<\rho_{1}<(l+4) \rho_{2},
$$

and for the $a b^{\prime}$ - and $a c$-sector one obtains

$$
l \rho_{2}<\rho_{1}<(l+4) \rho_{2} .
$$

With $l=7$, the last condition (2.17) is the stronger one and implies condition (2.16). Hence, once the conditions (2.14) and (2.17) are satisfied, all scalars in the sectors $a b$, $a c^{\prime}, a b^{\prime}$ and $a c$ are massive and supersymmetry is completely broken. The angles satisfying these conditions form a tetrahedron [16]. It is illustrated in figure 2, together with a domain of small angles.

The appearance of tachyons is a generic feature of non-supersymmetric intersecting D-brane models. However, it is argued that such tachyons can be removed by couplings to moduli fields that parametrize the distance between branes in tori where they are parallel. In the T-dual picture discussed in the following section these moduli correspond to Wilsonlines $\xi, \xi^{\prime}$ that acquire vacuum expectation values (see, for example, [16, 21]). In the present model the corresponding superpotential terms would have the form (in superfield notation, see table 2),

$$
W_{\xi, \xi^{\prime}}=\lambda_{1} \xi \mathbf{1}_{1,-1} \mathbf{1}_{-1,1}+\lambda_{2} \xi^{\prime} \mathbf{1}_{1,1} \mathbf{1}_{-1,-1} .
$$

Clearly, existence and stability of a ground state require an appropriate potential for $\xi$, $\xi^{\prime}$. At tree-level the potential is flat. To compute the one-loop quantum correction to the potential is an essential goal of this paper. To achieve this we first construct the Tdual type I string compactification on a magnetized torus, which allows a straightforward computation of the full mass spectrum of the model as well as Yukawa couplings. 

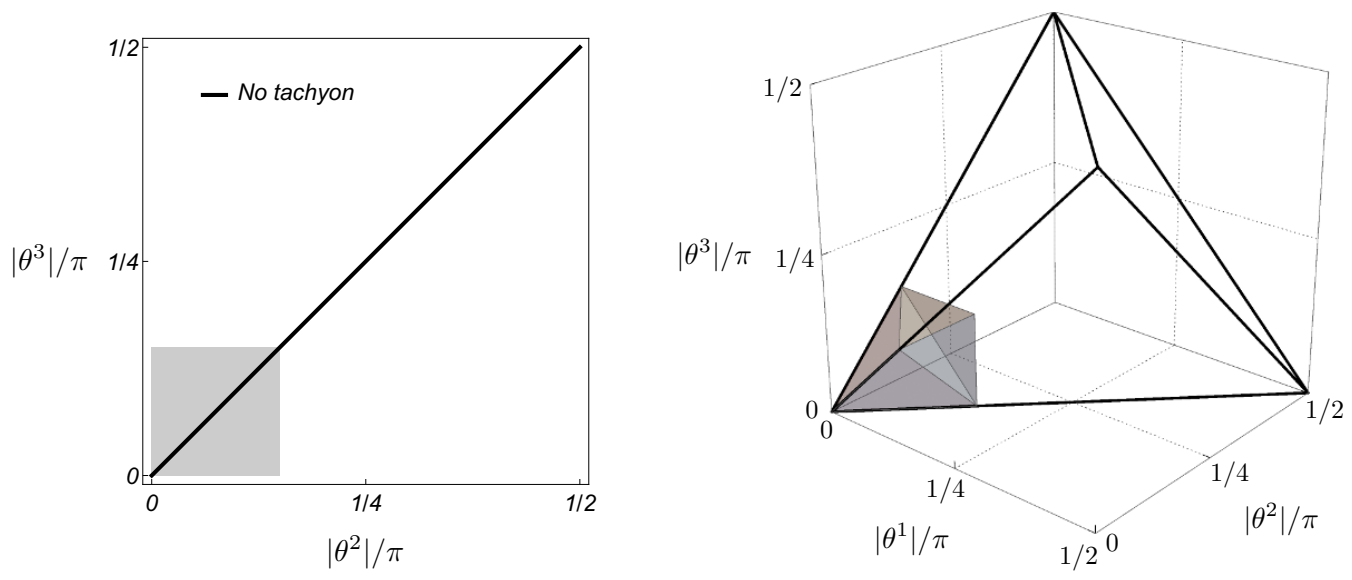

Figure 2. Domain of angles for which no tachyons appear. Left: line in $\theta^{2}-\theta^{3}$-plane in case of no flux in first torus, i.e. $\theta^{1}=0$. Right: tetrahedron in case of fluxes in all tori. The gray areas indicate small-angle domains with $\left|\theta^{i}\right| / \pi<0.15$.

\section{T-dual toroidal flux compactification}

The intersecting D-brane model constructed in the previous section is T-dual to a type I compactification on a magnetized dual rectangular torus $T_{1}^{2} \times T_{2}^{2} \times T_{3}^{2}$ with the identifications

$$
z_{i} \sim z_{i}+L_{i} / 2, \quad z_{i} \sim z_{i}+2 \pi^{2} \alpha^{\prime} / L_{i}^{\prime}
$$

where the angles $\theta_{e}^{i}$ between brane $e$ and the orientifold plane are related to magnetic flux densities in the 2-tori $T_{i}^{2}[13]$,

$$
\tan \theta_{e}^{i}=2 \pi \alpha^{\prime} g f_{I}^{i}
$$

Here $g$ is the gauge coupling, brane $e(e=a, b, c)$ has a $\mathrm{U}(1)$ group with Cartan generator $H_{I}(I=0,1,2)$, and $f_{I}^{i}$ is the corresponding flux density in the torus $T_{i}^{2}$. Using eq. (2.9), $\tan \theta_{e}^{i}=m_{I}^{i} \rho_{i}$, this implies the Dirac quantization condition for the flux densities $f_{I}^{i}$,

$$
g \int_{T_{i}^{2}} f_{I}^{i}=\frac{4 \pi^{2} \alpha^{\prime}}{\rho_{i}} g f_{I}^{i}=2 \pi m_{I}^{i} .
$$

For small angles, corresponding to small flux densities, one has ${ }^{2}$

$$
\theta_{e}^{i} \simeq m_{I}^{i} \rho_{i}=2 \pi \alpha^{\prime} g f_{I}^{i}, \quad \rho_{i}=L_{i}^{\prime} / L_{i}
$$

The considered D-brane model has three stacks of branes and therefore three U(1) factors, $\mathrm{U}(1)_{a}, \mathrm{U}(1)_{b}$ and $\mathrm{U}(1)_{c}$. Correspondingly, each torus $T_{i}^{2}$ can have three flux densities $f_{I}^{i}$, which allow to break $\mathrm{SO}(32)$ to the gauge group of the D-brane model,

$$
\mathrm{SO}(32) \supset \mathrm{U}(16) \supset \mathrm{U}(14) \times \mathrm{U}(1) \times \mathrm{U}(1) .
$$

\footnotetext{
${ }^{2}$ In the following we shall use the notations $f_{e}^{i}, m_{e}^{i}$ and $f_{I}^{i}, m_{I}^{i}$ in parallel, according to convenience.
} 
The corresponding decomposition of the adjoint representation reads (see appendices $\mathrm{A}$ and $\mathrm{B}, N=14$ ),

$$
\mathrm{SO}(32) \sim\left(\begin{array}{c|c|c|c|c|c}
\mathrm{U}(N) & N_{-1,0} & N_{0,-1} & A & N_{1,0} & N_{0,1} \\
a a & a b & a c & a a^{\prime} & a b^{\prime} & a c^{\prime} \\
\hline \bar{N}_{1,0} & \mathrm{U}(1) & 1_{1,-1} & N_{1,0} & 0 & 1_{1,1} \\
b a & b b & b c & b a^{\prime} & b b^{\prime} & b c^{\prime} \\
\hline \bar{N}_{0,1} & 1_{-1,1} & \mathrm{U}(1) & N_{0,1} & 1_{1,1} & 0 \\
c a & c b & c c & c a^{\prime} & c b^{\prime} & c c^{\prime} \\
\hline A^{*} & \bar{N}_{-1,0} & \bar{N}_{0,-1} & \mathrm{U}(N)^{*} & \bar{N}_{1,0} & \bar{N}_{0,1} \\
a^{\prime} a & a^{\prime} b & a^{\prime} c & a^{\prime} a^{\prime} & a^{\prime} b^{\prime} & a^{\prime} c^{\prime} \\
\hline \bar{N}_{-1,0} & 0 & 1_{-1,-1} & N_{-1,0} & \mathrm{U}(1)^{*} & 1_{-1,1} \\
b^{\prime} a & b^{\prime} b & b^{\prime} c & b^{\prime} a^{\prime} & b^{\prime} b^{\prime} & b^{\prime} c^{\prime} \\
\hline \bar{N}_{0,-1} & 1_{-1,-1} & 0 & N_{0,-1} & 1_{1,-1} & \mathrm{U}(1)^{*} \\
c^{\prime} a & c^{\prime} b & c^{\prime} c & c^{\prime} a^{\prime} & c^{\prime} b^{\prime} & c^{\prime} c^{\prime}
\end{array}\right) .
$$

Each block is labeled by the related brane intersection. The upper left and the lower right quadrant correspond to the adjoint representation of $\mathrm{U}(16)$, whereas the upper right and the lower left quadrant represent the antisymmetric representation of $\mathrm{U}(16)$, decomposed with respect to $\mathrm{U}(14) \times \mathrm{U}(1) \times \mathrm{U}(1)$.

The representation in the block ef feels the magnetic flux $f_{e f}^{i}=f_{e}^{i}-f_{f}^{i}$ in torus $T_{i}^{2}$. According to the index theorem the multiplicities of chiral zero-modes are given by

$$
I_{e f}=\left(\frac{g}{2 \pi}\right)^{3} \prod_{i} \int_{T_{i}^{2}} f_{e f}^{i}=\prod_{i}\left(m_{e}^{i}-m_{f}^{i}\right)
$$

Because of eq. (3.3) these multiplicities agree with the intersection numbers of the D-brane model given in table 2 .

The starting point for the computation of the $4 \mathrm{~d}$ effective action is the $10 \mathrm{~d}$ Super-YangMills action with $\mathcal{N}=4$ supersymmetry and gauge group $\mathrm{SO}(32)$, which is conveniently expressed in term of $4 \mathrm{~d}$ vector superfields $V$ and chiral superfields $\phi$ [27, 28],

$$
\begin{aligned}
S_{10}= & \int d^{10} x\left\{\frac{1}{k} \int d^{2} \theta \operatorname{tr}\left[\frac{1}{4} W W+\frac{1}{2} \varepsilon_{i j k} \phi^{i}\left(\partial_{j} \phi^{k}+\frac{g}{3 \sqrt{2}}\left[\phi^{j}, \phi^{k}\right]\right)\right]+\right.\text { h.c. } \\
& \left.+\frac{1}{k} \int d^{4} \theta \frac{1}{g^{2}} \operatorname{tr}\left[\left(-\sqrt{2} \bar{\partial}_{i}+g \bar{\phi}^{i}\right) e^{g V}\left(\sqrt{2} \partial_{i}+g \phi^{i}\right) e^{-g V}+\bar{\partial}_{i} e^{g V} \partial_{i} e^{-g V}\right]\right\} .
\end{aligned}
$$

Here $W$ is the field strength of the vector field, ${ }^{3} i, j, k=1,2,3$ label the three 2-tori, and our trace convention is $\operatorname{tr}\left(T_{a} T_{b}\right)=k \delta_{a b}$. Expanding the exponentials, integrating some of

\footnotetext{
${ }^{3}$ We use the conventions of [32], and we have dropped the WZW term that vanishes in WZ gauge, $V^{3}=0$.
} 
the terms by part, and using the WZ gauge $V^{3}=0$, one obtains

$$
\begin{aligned}
S_{10}=\int d^{10} x\left\{\frac{1}{k} \int d^{2} \theta \operatorname{tr}\left[\frac{1}{4} W W+\frac{1}{2} \varepsilon_{i j k} \phi^{i}\left(\partial_{j} \phi^{k}+\frac{g}{3 \sqrt{2}}\left[\phi^{j}, \phi^{k}\right]\right)\right]+\right.\text { h.c. } \\
+\frac{1}{k} \int d^{4} \theta \operatorname{tr}\left[\bar{\phi}^{i} \phi^{i}+\sqrt{2}\left(\partial_{i} \bar{\phi}^{i}+\bar{\partial}_{i} \phi^{i}\right) V-g\left[\bar{\phi}^{i}, \phi^{i}\right] V\right. \\
\left.\left.+\left(\bar{\partial}_{i} V-\frac{g}{\sqrt{2}}\left[\bar{\phi}^{i}, V\right]\right)\left(\partial_{i} V+\frac{g}{\sqrt{2}}\left[\phi^{i}, V\right]\right)\right]\right\} .
\end{aligned}
$$

Note, that in this action the invariance with respect to 4 supersymmetry transformations is manifest whereas the invariance with respect to 12 further supersymmetry transformations is hidden. This will be important in our discussion of supersymmetry breaking by magnetic fluxes in the following sections.

Vector and chiral superfields are conveniently decomposed into the different sectors indicated in eq. (3.6). The unbroken group is $H=\mathrm{U}(N) \times \mathrm{U}(1) \times \mathrm{U}(1) \subset \mathrm{U}(N+2)$ with the $\mathrm{U}(1)$ and $\mathrm{SU}(N)$ generators ${ }^{4}$

$$
H_{0}=\frac{1}{\sqrt{N}} T_{\alpha \alpha}, \quad H_{1}=T_{N+1, N+1}, \quad H_{2}=T_{N+2, N+2}, \quad \tilde{T}_{\alpha \beta} .
$$

In terms of the generators of $H$ and $\mathrm{SO}(32) / H$, vector superfields can be expressed as (see appendix B)

$$
\begin{aligned}
V= & V_{\alpha \beta} \tilde{T}_{\alpha \beta}+V_{I} H_{I}+V_{\alpha}^{-0} T_{\alpha}^{-0}+V_{\alpha}^{+0} T_{\alpha}^{+0}+V_{\alpha}^{0-} T_{\alpha}^{0-}+V_{\alpha}^{0+} T_{\alpha}^{0+} \\
& +V^{+-} T^{+-}+V^{-+} T^{-+}+\frac{1}{2} V_{\gamma \delta}^{+} X_{\gamma \delta}^{+}+\frac{1}{2} V_{\gamma \delta}^{-} X_{\gamma \delta}^{-}+\tilde{V}_{\alpha}^{+0} X_{\alpha}^{+0} \\
& +\tilde{V}_{\alpha}^{-0} X_{\alpha}^{-0}+\tilde{V}_{\alpha}^{0+} X_{\alpha}^{0+}+\tilde{V}_{\alpha}^{0-} X_{\alpha}^{0-}+V^{++} X^{+-}+V^{--} X^{--}
\end{aligned}
$$

The charges with respect to $H_{1}$ and $H_{2}$ are indicated explicitly. The fields $V_{\alpha}^{-0}, V_{\alpha}^{0-}, \tilde{V}_{\alpha}^{+0}$ and $\tilde{V}^{0+}$ transform in the fundamental, and the fields $V_{\alpha}^{+0}, V_{\alpha}^{0+}, \tilde{V}_{\alpha}^{-0}$ and $\tilde{V}^{0-}$ in the anti-fundamental representation of $\mathrm{SU}(N)$, respectively. $V_{\gamma \delta}^{+}$is an antisymmetric tensor of $\mathrm{SU}(N)$ and $V_{\gamma \delta}^{-}$is the complex conjugate representation. $V_{\gamma \delta}^{ \pm}$are neutral with respect to $H_{1}$ and $H_{2}$. Here, the superscript denotes the charge with respect to $H_{0}$. Analogously, the decomposition of the chiral and antichiral superfields is given by ${ }^{5}$

$$
\begin{aligned}
\phi= & \phi_{\alpha \beta} \tilde{T}_{\alpha \beta}+\chi_{I} H_{I}+\phi_{\alpha}^{-0} T_{\alpha}^{-0}+\phi_{\alpha}^{+0} T_{\alpha}^{+0}+\phi_{\alpha}^{0-} T_{\alpha}^{0-}+\phi_{\alpha}^{0+} T_{\alpha}^{0+} \\
& +\phi^{+-} T^{+-}+\phi^{-+} T^{-+}+\frac{1}{2} \phi_{\gamma \delta}^{+} X_{\gamma \delta}^{+}+\frac{1}{2} \phi_{\gamma \delta}^{-} X_{\gamma \delta}^{-}+\tilde{\phi}_{\alpha}^{+0} X_{\alpha}^{+0} \\
& +\tilde{\phi}_{\alpha}^{-0} X_{\alpha}^{-0}+\tilde{\phi}_{\alpha}^{0+} X_{\alpha}^{0+}+\tilde{\phi}_{\alpha}^{0-} X_{\alpha}^{0-}+\phi^{++} X^{++}+\phi^{--} X^{--} \\
\bar{\phi}= & \bar{\phi}_{\alpha \beta} \tilde{T}_{\beta \alpha}+\bar{\chi}_{I} H_{I}+\bar{\phi}_{\alpha}^{-0} T_{\alpha}^{+0}+\bar{\phi}_{\alpha}^{+0} T_{\alpha}^{-0}+\bar{\phi}_{\alpha}^{0-} T_{\alpha}^{0+}+\bar{\phi}_{\alpha}^{0+} T_{\alpha}^{0-} \\
& +\bar{\phi}^{+-} T^{-+}+\bar{\phi}^{-+} T^{+-}+\frac{1}{2} \bar{\phi}_{\gamma \delta}^{+} X_{\gamma \delta}^{-}+\frac{1}{2} \bar{\phi}_{\gamma \delta}^{-} X_{\gamma \delta}^{+}+\overline{\tilde{\phi}}_{\alpha}^{+0} X_{\alpha}^{-0} \\
& +\overline{\tilde{\phi}}_{\alpha}^{-0} X_{\alpha}^{+0}+\overline{\tilde{\phi}}_{\alpha}^{0+} X_{\alpha}^{0-}+\overline{\tilde{\phi}}_{\alpha}^{0-} X_{\alpha}^{0+}+\bar{\phi}^{++} X^{--}+\bar{\phi}^{--} X^{++}
\end{aligned}
$$

\footnotetext{
${ }^{4} \mathrm{~A}$ sum over repeated indices is understood.

${ }^{5}$ Note that $\bar{\phi}_{\alpha}^{-0}$ stands for $\overline{\phi_{\alpha}^{-0}}$.
} 
In order to compute the mass spectrum caused by the magnetic fluxes and also for a discussion of tachyon condensation one has to know the Yukawa couplings of the model. They are obtained from the cubic gauge coupling in the action (3.9) and the commutators listed in appendix B. A straightforward calculation yields the result

$$
\mathcal{L}_{\mathrm{Y}}=\frac{1}{k} \int d^{2} \theta \frac{g}{6 \sqrt{2}} \varepsilon_{i j k} \operatorname{tr}\left[\phi^{i}\left[\phi^{j}, \phi^{k}\right]\right]=\frac{g}{\sqrt{2}} \varepsilon_{i j k} \int d^{2} \theta\left(W_{i j k}^{1}+W_{i j k}^{2}\right),
$$

where $W^{1}$ and $W^{2}$ describe couplings without and with $\mathrm{SU}(N)$ fields, respectively,

$$
\begin{aligned}
W_{i j k}^{1}= & \frac{1}{\sqrt{N}} \chi_{0}^{i}\left(\phi_{\alpha}^{j-0} \phi_{\alpha}^{k+0}+\phi_{\alpha}^{j 0-} \phi_{\alpha}^{k 0+}-\tilde{\phi}_{\alpha}^{j+0} \tilde{\phi}_{\alpha}^{k-0}-\tilde{\phi}_{\alpha}^{j 0+} \tilde{\phi}_{\alpha}^{k 0-}+\phi_{\alpha \beta}^{j+} \phi_{\alpha \beta}^{k-}\right) \\
& +\chi_{1}^{i}\left(-\phi_{\alpha}^{j-0} \phi_{\alpha}^{k+0}-\tilde{\phi}_{\alpha}^{j+0} \tilde{\phi}_{\alpha}^{k-0}+\phi^{j+-} \phi^{k-+}+\tilde{\phi}^{j++} \tilde{\phi}^{k--}\right) \\
& +\chi_{2}^{i}\left(-\phi_{\alpha}^{j 0-} \phi_{\alpha}^{k 0+}-\tilde{\phi}_{\alpha}^{j 0+} \tilde{\phi}_{\alpha}^{k 0-}-\phi^{j+-} \phi^{k-+}+\tilde{\phi}^{j++} \tilde{\phi}^{k--}\right) \\
& -\phi^{i+-}\left(\phi_{\alpha}^{j-0} \phi_{\alpha}^{k 0+}+\tilde{\phi}_{\alpha}^{j 0+} \tilde{\phi}_{\alpha}^{k-0}\right)-\phi^{i-+}\left(\phi_{\alpha}^{j 0-} \phi_{\alpha}^{k+0}+\tilde{\phi}_{\alpha}^{j+0} \tilde{\phi}_{\alpha}^{k 0-}\right) \\
& +\phi^{i++}\left(-\phi_{\alpha}^{j-0} \tilde{\phi}_{\alpha}^{k 0-}+\phi_{\alpha}^{j 0-} \tilde{\phi}_{\alpha}^{k-0}\right)+\phi^{i--}\left(\phi_{\alpha}^{j+0} \tilde{\phi}_{\alpha}^{k 0+}-\phi_{\alpha}^{j 0+} \tilde{\phi}_{\alpha}^{k+0}\right) \\
& -\phi_{\alpha \beta}^{i+}\left(\phi_{\beta}^{j+0} \tilde{\phi}_{\alpha}^{k-0}+\phi_{\beta}^{j 0+} \tilde{\phi}_{\alpha}^{k 0-}\right)-\phi_{\alpha \beta}^{i-}\left(\phi_{\beta}^{j-0} \tilde{\phi}_{\alpha}^{k+0}+\phi_{\beta}^{j 0-} \tilde{\phi}_{\alpha}^{k 0+}\right), \\
W_{i j k}^{2}= & \phi_{\alpha \beta}^{i}\left(\phi_{\beta}^{j-0} \phi_{\alpha}^{k+0}+\phi_{\beta}^{j 0-} \phi_{\alpha}^{k 0+}-\tilde{\phi}_{\beta}^{j+0} \tilde{\phi}_{\alpha}^{k-0}-\tilde{\phi}_{\beta}^{j 0+} \tilde{\phi}_{\alpha}^{k 0-}\right. \\
& \left.+\phi_{\gamma \beta}^{j+} \phi_{\gamma \alpha}^{k-}-\phi_{\gamma \alpha}^{j-} \phi_{\gamma \beta}^{k+}+\phi_{\beta \gamma}^{j} \phi_{\gamma \alpha}^{k}-\phi_{\gamma \alpha}^{j} \phi_{\beta \gamma}^{k}\right) .
\end{aligned}
$$

Note, that these couplings involve 10d fields. The 4d effective Lagrangian is obtained by performing a mode expansion for all fields and by evaluating the overlap integrals of products of mode functions.

The gauge group $\mathrm{SO}(32)$ is broken to the subgroup $\mathrm{U}(N) \times \mathrm{U}(1) \times \mathrm{U}(1)$ by a background of the $\mathrm{U}(1)$ gauge fields in the compact dimensions,

$$
\left\langle\chi_{I}^{i}\right\rangle=\frac{1}{\sqrt{2}} f_{I}^{i} \bar{z}_{i}+\xi_{I}^{i}
$$

corresponding to Wilson lines and magnetic fluxes in the three 2 -tori $\left(\left.\chi_{I}^{i}\right|_{\theta=\bar{\theta}=0}=\left(A_{I, 3+2 i}+\right.\right.$ $\left.\left.i A_{I, 2+2 i}\right) / \sqrt{2}\right)$,

$$
\left\langle F_{2+2 i, 3+2 i}\right\rangle \delta_{i j}=\bar{\partial}_{i}\left\langle\chi_{I}^{j}\right\rangle=f_{I}^{i} \delta_{i j} .
$$

The mass spectrum of the charged fields is obtained by calculating the quadratic part of the effective action in this gauge field background.

Each pair of fields in eq. (3.6), such as $\left(A, A^{*}\right),\left(N_{-1,0}, \bar{N}_{1,0}\right)$ etc., feels magnetic fluxes $f_{I}^{i}$ in the three tori. The mass spectrum of each sector ef is then characterized by Landau levels $\left(n_{1}, n_{2}, n_{3}\right)$ and internal helicities $\left(\sigma_{1}, \sigma_{2}, \sigma_{3}\right)$ in the three tori, with $n_{i} \in \mathbb{N}$ and $\sigma_{i}=0, \pm 1 / 2, \pm 1$. Hence, for each triple of Landau levels one obtains two $4 \mathrm{~d}$ complex vector states, eight $4 \mathrm{~d}$ Weyl fermions and six complex $4 \mathrm{~d}$ scalars. Their masses have been obtained in a type I string compactification on a magnetized torus $\left(f_{e f}^{i}=f_{e}^{i}-f_{f}^{i}\right)[9]$,

$$
M_{e f}^{2}(n ; \sigma)=g \sum_{i}\left(\left(2 n_{i}+1\right)\left|f_{e f}^{i}\right|+2 f_{e f}^{i} \sigma_{i}\right)
$$


Here $\sigma=\left(\sigma_{1}, \sigma_{2}, \sigma_{3}\right)$ takes the values $(0,0,0),( \pm 1 / 2, \pm 1 / 2, \pm 1 / 2)$ and $( \pm, 0,0),(0, \pm, 0)$, $(0,0, \pm)$ for vectors, fermions and scalars, respectively. Contrary to what one might expect, these masses are not associated with a single set $\left(n_{1}, n_{2}, n_{3}\right)$ of Landau levels in a mode expansion of the 10d fields in eq. (3.6). As we shall see in the following section, the magnetic fluxes mix neighboring levels in the Kaluza-Klein towers, and mass eigenstates are linear combinations of different Landau levels.

In this T-dual internal magnetic field description that we will mainly focus on, supersymmetry breaking for generic magnetic fields is captured by the (internal helicity) spin-magnetic field coupling in the mass formula (3.19). The special values of magnetic fields for which some supersymmetry is preserved can be understood in various ways. One of them is by checking the boson and fermion mass formulae and the flux value parameters for which there is boson-fermion degeneracy. Equivalently, the scalar potential that we will compute in section 6 will turn out to vanish precisely for these flux values. Another way to understand supersymmetry breaking and preservation is by writing the gaugino variation for the Super-Yang-Mills theory directly in ten dimension, before compactification, which reads

$$
\delta \lambda^{a}=-\frac{1}{4} \Gamma^{P Q} F_{P Q}^{a} \epsilon,
$$

where $\Gamma^{P Q}=\frac{1}{2}\left(\Gamma^{P} \Gamma^{Q}-\Gamma^{Q} \Gamma^{P}\right), F_{P Q}^{a}$ is the 10d Yang-Mills field strength and $\epsilon$ are the $10 \mathrm{~d}$ supersymmetry parameters. The number of preserved supercharges is given by the number of independent spinors $\epsilon$ annihilated by the operator

$$
\Gamma(f)=\Gamma^{P Q}\left\langle F_{P Q}^{I}\right\rangle \sim \Gamma^{45} f_{1}+\Gamma^{67} f_{2}+\Gamma^{89} f_{3},
$$

where we defined the fluxes $f_{k}=\left\langle F_{2+2 k, 3+2 k}\right\rangle, k=1,2,3$, and $I$ is a Cartan subalgebra generator supporting the magnetic flux. A well known and convenient Fock space basis for fermions is obtained by introducing the creation and annihilation operators (see, for example, [33])

$$
b_{k}^{\dagger}=\frac{1}{2}\left(\Gamma^{2+2 k}-i \Gamma^{3+2 k}\right), \quad b_{k}=\frac{1}{2}\left(\Gamma^{2+2 k}+i \Gamma^{3+2 k}\right),
$$

with $k=1,2,3$ denoting the complex internal space degrees of freedom. Using

$$
b_{k}^{\dagger} b_{k}-b_{k} b_{k}^{\dagger}=i \Gamma^{2+2 k} \Gamma^{3+2 k}=-2 J_{2+2 k, 3+2 k},
$$

where $J_{2+2 k, 3+2 k}$ are rotations generators in the internal space, one can rewrite the operator $\Gamma$ as

$$
\Gamma(f) \sim \sum_{k=1}^{3} f_{k}\left(b_{k}^{\dagger} b_{k}-b_{k} b_{k}^{\dagger}\right) .
$$

Then, by explicit construction, one can show that $\Gamma(f) \epsilon=0$ for

$$
\begin{array}{llllr}
\epsilon_{0}=|0\rangle & \text { and } & b_{1}^{\dagger} b_{2}^{\dagger} b_{3}^{\dagger}|0\rangle, & \text { if } & f_{1}+f_{2}+f_{3}=0, \\
\epsilon_{1}=b_{1}^{\dagger}|0\rangle & \text { and } & b_{2}^{\dagger} b_{3}^{\dagger}|0\rangle, & \text { if } & -f_{1}+f_{2}+f_{3}=0, \\
\epsilon_{2}=b_{2}^{\dagger}|0\rangle & \text { and } & b_{3}^{\dagger} b_{1}^{\dagger}|0\rangle, & \text { if } & f_{1}-f_{2}+f_{3}=0, \\
\epsilon_{3}=b_{3}^{\dagger}|0\rangle & \text { and } & b_{1}^{\dagger} b_{2}^{\dagger}|0\rangle, & \text { if } & f_{1}+f_{2}-f_{3}=0 .
\end{array}
$$


These relations match the field theory limit of the intersecting brane supersymmetry conditions (2.12). As we will see explicitly in the following sections, the effective theory does not easily capture the supersymmetry restoration points in moduli space. The reason is that the supercharge corresponding to $\epsilon_{0}$ is aligned with the superspace expansion, whereas the other preserved supercharges corresponding to $\epsilon_{1,2,3}$ are not, and the corresponding supersymmetries are hidden in an effective Lagrangian that at first sight looks non-supersymmetric.

In later sections we will discuss tachyon condensation, which requires to add the fluctuations around the magnetic background (3.21). In this case, the operator $\Gamma$ is changed according to

$$
\begin{aligned}
\Gamma \equiv \Gamma^{P Q} F_{P Q}^{I}= & 2 \sum_{k=1}^{3}\left(c_{A B}^{I} \phi_{A}^{k} \bar{\phi}_{B}^{k}-i f_{I}^{k}\right)\left(b_{k}^{\dagger} b_{k}-b_{k} b_{k}^{\dagger}\right) \\
& +4 c_{A B}^{I} \sum_{i<j}\left[b_{i}^{\dagger} b_{j}^{\dagger} \phi_{A}^{i} \phi_{B}^{j}+b_{i} b_{j} \bar{\phi}_{A}^{i} \bar{\phi}_{B}^{j}+b_{i}^{\dagger} b_{j} \phi_{A}^{i} \bar{\phi}_{B}^{j}+b_{i} b_{j}^{\dagger} \bar{\phi}_{A}^{i} \phi_{B}^{j}\right],
\end{aligned}
$$

where $c_{A B}^{I}$ are the structure constants of the 10d Yang-Mills gauge group and $A, B$ are indices of the adjoint representation. Acting with the operator $\Gamma$ on the spinors $Q \equiv$ $\left(\epsilon_{0}, \epsilon_{1}, \epsilon_{2}, \epsilon_{3}\right)^{T}$ one defines a $4 \times 4$ matrix $\mathcal{M}$ according to

$$
\Gamma Q=\mathcal{M} Q
$$

Notice that the spinors $\epsilon_{0}, \epsilon_{i}$ do not carry flux charge, since they transform only under the $\mathrm{SU}(4) \mathrm{R}$-symmetry group, which commutes with the gauge group generators. They should be understood as the constant zero modes of the KK reduction from $10 \mathrm{~d}$ to $4 \mathrm{~d}$. Labeling the four rows and columns by $0,1,2,3$, the matrix elements are computed to be

$$
\begin{aligned}
& \mathcal{M}_{00}=-2 i\left(f_{I}^{1}+f_{I}^{2}+f_{I}^{3}\right)-2 c_{A B}^{I} \bar{\phi}_{A}^{k} \phi_{B}^{k}, \mathcal{M}_{0 i}=-4 c_{A B}^{I} \epsilon_{i j k} \bar{\phi}_{A}^{j} \bar{\phi}_{B}^{k}, \\
& \mathcal{M}_{i 0}=4 c_{A B}^{I} \epsilon_{i j k} \phi_{A}^{j} \phi_{B}^{k}, \mathcal{M}_{i j}=2 i\left(f_{I}^{1}+f_{I}^{2}+f_{I}^{3}-2 f_{I}^{i}\right) \delta_{i j}+2 c_{A B}^{I}\left(\bar{\phi}_{A}^{k} \phi_{B}^{k} \delta_{i j}-2 \bar{\phi}_{A}^{i} \phi_{B}^{j}\right) .
\end{aligned}
$$

After compactification to four dimensions, quantities like $\phi_{A}^{j} \bar{\phi}_{B}^{k}$ should be understood as integrated over the internal space, leading to a sum over Landau levels $\sum_{n, n^{\prime}} \phi_{A, n n^{\prime}}^{j} \bar{\phi}_{B, n n^{\prime}}^{k}$. As before, the number of zero eigenvalues of the matrix $\mathcal{M}$ is the number of unbroken supersymmetries in four dimensions. Let us study some simple examples:

- One flux, say $f_{I}^{1}=f_{I}^{2}=0, f_{I}^{3} \neq 0$. In the absence of vev's for $\phi$ 's, there is no zero eigenvalue according to (3.25) and all supersymmetries are broken. However, by giving a vacuum expectation value $\phi_{3} \neq 0$, one can set to zero all matrix elements and restore full $\mathcal{N}=4$ supersymmetry by choosing $c_{A B}^{I} \phi_{A}^{3} \bar{\phi}_{B}^{3}=i f_{I}^{3}$. Notice that the vev's concern fields charged under the (Cartan) generator $H_{I}$.

- Two fluxes, say $f_{I}^{1}=0, f_{I}^{2}, f_{I}^{3} \neq 0$. In this case, in the absence of vev's for $\phi$ 's, all supersymmetries are generically broken, except for $f_{I}^{2}= \pm f_{I}^{3}$, which preserves $\mathcal{N}=2$ supersymmetry. For $f_{I}^{2} \neq f_{I}^{3}$, one can easily find vev's restoring $\mathcal{N}=2$ supersymmetry:

$$
c_{A B}^{I} \bar{\phi}_{A}^{2} \phi_{B}^{3}=0, i\left(f_{I}^{2}-f_{I}^{3}\right)=2 c_{A B}^{I}\left(\bar{\phi}_{A}^{2} \phi_{B}^{2}-\bar{\phi}_{A}^{3} \phi_{B}^{3}\right),
$$


which can be satisfied for example for $\phi_{B}^{2}=0$ and $i\left(f_{I}^{2}-f_{I}^{3}\right)=-2 c_{A B}^{I} \bar{\phi}_{A}^{3} \phi_{B}^{3}$. One can also search the existence of an $\mathcal{N}=4$ vacuum. It seems natural to assume $\phi_{A}^{1}=0$, both since this field is not tachyonic for such fluxes and since in this case the matrix $\mathcal{M}$ has a simpler block-diagonal form of two $2 \times 2$ matrices. The conditions for the existence of an $\mathcal{N}=4$ vacuum are

$$
\begin{array}{ll}
\operatorname{tr}\left[\phi^{2}, \bar{\phi}^{2}\right] H_{I}=-f_{I}^{2}, & \operatorname{tr}\left[\phi^{3}, \bar{\phi}^{3}\right] H_{I}=-f_{I}^{3}, \\
\operatorname{tr}\left[\phi^{2}, \bar{\phi}^{3}\right] H_{I}=0, & \operatorname{tr}\left[\phi^{2}, \phi^{3}\right] H_{I}=0 .
\end{array}
$$

- Three fluxes, say $f_{I}^{1}, f_{I}^{2}, f_{I}^{3} \neq 0$. In this case, in the absence of vev's for $\phi$ 's, all supersymmetries are generically broken, except for $f_{I}^{1} \pm f_{I}^{2} \pm f_{I}^{3}=0$, which preserves $\mathcal{N}=1$ supersymmetry. For $f_{I}^{1} \pm f_{I}^{2} \pm f_{I}^{3} \neq 0$, one can easily find vev's restoring $\mathcal{N}=1$ supersymmetry by switching on only one vev. For example, one can choose $\phi^{2}=\phi^{3}=0$ and

$$
i\left(f_{I}^{1} \pm f_{I}^{2} \pm f_{I}^{3}\right)=c_{A B}^{I} \phi_{A}^{1} \bar{\phi}_{B}^{1},
$$

for any (single) choice of signs. The case of vev's restoring more supersymmetries seems similar to the previous example with two fluxes. In order to obtain $\mathcal{N}=4$ supersymmetry, one would need vev's for the three $\phi^{i}$ 's and satisfy

$$
\operatorname{tr}\left[\phi^{i}, \bar{\phi}^{i}\right] H_{I}=-f_{I}^{i}, \epsilon_{i j k} \operatorname{tr}\left[\phi^{i}, \phi^{j}\right] H_{I}=0, \epsilon_{i j k} \operatorname{tr}\left[\phi^{i}, \bar{\phi}^{j}\right] H_{I}=0 .
$$

This seems always possible.

In all cases, one should also impose the D-term conditions for the charged generators. They are more complicated than the ones for the Cartan generators written above. The reason is that in addition to bilinear terms similar to the ones for Cartan generators (for example, $\epsilon_{i j k} \operatorname{tr}\left[\phi^{i}, \bar{\phi}^{j}\right] E_{\alpha}$ ), there are also terms linear in the charged fields coming from the covariant derivative acting on charged fields, which have a non-constant profile in the internal space. These terms, of the type $\sqrt{f_{I}^{i}} a_{\alpha}^{i} \operatorname{tr}\left(\phi^{j} E_{\alpha}\right)$ or $\sqrt{f_{I}^{i}} a_{\alpha}^{i \dagger} \operatorname{tr}\left(\phi^{j} E_{\alpha}\right)$, depending on the sign of the flux, can be computed in explicit cases and will be displayed explicitly in section 7. However, a general expression for these terms, and a general analysis of the charged D-term conditions is beyond the scope of this paper. Therefore, at this point we leave open the question whether or not there are vacua with full $\mathcal{N}=4$ supersymmetry in the case of arbitrary fluxes.

\section{Matter sector}

In this section we consider potentially tachyon-free sectors of the model, i.e., the antisymmetric tensor with vector-like massless fermions, and the fields in fundamental and anti-fundamental representations with chiral fermions.

\subsection{Antisymmetric tensor $\left(a a^{\prime}\right.$-sector $)$}

Let us start with the antisymmetric tensor fields, $V_{\gamma \delta}^{ \pm}, \phi_{\gamma \delta}^{ \pm}$and $\bar{\phi}_{\gamma \delta}^{ \pm}$. These fields have charge $\pm 2 / \sqrt{N}$ with respect to $H_{0}$ and charge zero with respect to $H_{1}$ and $H_{2}$. For simplicity, we 
choose $\xi_{0}^{i}=0$. According to table 1 , the flux in the first torus vanishes and the fluxes in the second and third torus satisfy the quantization conditions $\left(\rho_{i}=L_{i}^{\prime} / L_{i}\right)$,

$$
\frac{g}{2 \pi \sqrt{N}} \int_{T_{2}^{2}} f_{0}^{2}=\frac{g}{\sqrt{N}} \frac{2 \pi \alpha^{\prime}}{\rho_{2}} f_{0}^{2}=2, \quad \frac{g}{2 \pi \sqrt{N}} \int_{T_{3}^{2}} f_{0}^{3}=\frac{g}{\sqrt{N}} \frac{2 \pi \alpha^{\prime}}{\rho_{3}} f_{0}^{3}=1,
$$

which yields the flux densities

$$
g f_{0}^{2}=2 \sqrt{N} \frac{\rho_{2}}{2 \pi \alpha^{\prime}} \equiv g \sqrt{N} f_{2}, \quad g f_{0}^{3}=\sqrt{N} \frac{\rho_{3}}{2 \pi \alpha^{\prime}} \equiv g \sqrt{N} f_{3} .
$$

For the special choice $\rho_{3}=2 \rho_{2}$ in eq. (2.14), the flux density is the same in both tori, i.e., $f_{0}^{2}=f_{0}^{3}$ or $f_{2}=f_{3}$.

Using the relevant commutators in eq. (B.7),

$$
\begin{aligned}
{\left[H_{0}, X_{\alpha \beta}^{ \pm}\right] } & = \pm \frac{2}{\sqrt{N}} X_{\alpha \beta}^{ \pm} \\
{\left[X_{\alpha \beta}^{+}, X_{\gamma \delta}^{-}\right] } & =\frac{2}{\sqrt{N}}\left(\delta_{\alpha \gamma} \delta_{\beta \delta}-\delta_{\beta \gamma} \delta_{\alpha \delta}\right)+\ldots
\end{aligned}
$$

it is straightforward to derive the quadratic part and the cubic couplings involving the neutral fields $\chi_{0}^{i} \equiv \chi^{i}$ and $\bar{\chi}_{0}^{i} \equiv \bar{\chi}^{i}$,

$$
\begin{aligned}
& S_{10} \supset \int d^{10} x\left\{\int d ^ { 2 } \theta \left(\frac{1}{4} W_{0} W_{0}\right.\right.\left.+\frac{1}{4} W_{\gamma \delta}^{+} W_{\gamma \delta}^{-}+\frac{1}{2} \varepsilon_{i j k} \phi_{\gamma \delta}^{i+}\left(\partial_{j}-\frac{\sqrt{2} g}{\sqrt{N}} \chi^{j}\right) \phi_{\gamma \delta}^{k-}\right)+ \text { h.c. } \\
&+\int d^{4} \theta\left(\bar{\chi}^{i} \chi^{i}+\frac{1}{2} \bar{\phi}_{\gamma \delta}^{i+} \phi_{\gamma \delta}^{i+}+\frac{1}{2} \bar{\phi}_{\gamma \delta}^{i-} \phi_{\gamma \delta}^{i-}+\sqrt{2}\left(\partial_{i} \bar{\chi}^{i}+\bar{\partial}_{i} \chi^{i}\right) V_{0}\right. \\
&+\frac{g}{\sqrt{N}}\left(\bar{\phi}_{\gamma \delta}^{i+} \phi_{\gamma \delta}^{i+}-\bar{\phi}_{\gamma \delta}^{i-} \phi_{\gamma \delta}^{i-}\right) V_{0} \\
&+\frac{1}{\sqrt{2}}\left(\left(\partial_{i}-\frac{\sqrt{2} g}{\sqrt{N}} \chi^{i}\right) \bar{\phi}_{\gamma \delta}^{i+}+\left(\bar{\partial}_{i}+\frac{\sqrt{2} g}{\sqrt{N}} \bar{\chi}^{i}\right) \phi_{\gamma \delta}^{i-}\right) V_{\gamma \delta}^{+} \\
&+\frac{1}{\sqrt{2}}\left(\left(\partial_{i}+\frac{\sqrt{2} g}{\sqrt{N}} \chi^{i}\right) \bar{\phi}_{\gamma \delta}^{i-}+\left(\bar{\partial}_{i}-\frac{\sqrt{2} g}{\sqrt{N}} \bar{\chi}^{i}\right) \phi_{\gamma \delta}^{i+}\right) V_{\gamma \delta}^{-} \\
&+\frac{1}{2}\left(\bar{\partial}_{i}+\frac{\sqrt{2} g}{\sqrt{N}} \bar{\chi}^{i}\right) V_{\gamma \delta}^{-}\left(\partial_{i}+\frac{\sqrt{2} g}{\sqrt{N}} \chi^{i}\right) V_{\gamma \delta}^{+} \\
&\left.\left.+\frac{1}{2}\left(\bar{\partial}_{i}-\frac{\sqrt{2} g}{\sqrt{N}} \bar{\chi}^{i}\right) V_{\gamma \delta}^{+}\left(\partial_{i}-\frac{\sqrt{2} g}{\sqrt{N}} \chi^{i}\right) V_{\gamma \delta}^{-}\right)\right\} .
\end{aligned}
$$

There is no $H_{0}$ flux in the first torus. To obtain the lowest mass eigenstates, we can therefore neglect the dependence of the fields on $z_{1}$. Inserting the background flux in the second and third torus yields covariant derivatives $\partial_{2} \pm 2 g f_{2} \bar{z}_{2}, \bar{\partial}_{2} \pm 2 g f_{2} z_{2}$, and $\partial_{3} \pm g f_{3} \bar{z}_{3}$, $\bar{\partial}_{3} \pm g f_{3} z_{3}$, which form a harmonic oscillator algebra. The fields can therefore be expanded in the corresponding set of orthonormal eigenfunctions. 
For a flux density $g f=2 \pi M, M \in \mathbb{N}$, one defines two pairs of annihilation and creation operators [24],

$$
\begin{array}{ll}
a_{+}=\frac{i}{\sqrt{2 g f}}(\partial+g f \bar{z}), & a_{+}^{\dagger}=\frac{i}{\sqrt{2 g f}}(\bar{\partial}-g f z), \\
a_{-}=\frac{i}{\sqrt{2 g f}}(\bar{\partial}+g f z), & a_{-}^{\dagger}=\frac{i}{\sqrt{2 g f}}(\partial-g f \bar{z}),
\end{array}
$$

which satisfy the commutation relations

$$
\left[a_{ \pm}, a_{ \pm}^{\dagger}\right]=1, \quad\left[a_{ \pm}, a_{\mp}\right]=0, \quad\left[a_{ \pm}, a_{\mp}^{\dagger}\right]=0 .
$$

The ground state wave functions are determined by

$$
a_{+} \xi_{0, j}=0, \quad a_{-} \bar{\xi}_{0, j}=0,
$$

where $j=0, \ldots|M|-1$ labels the degeneracy. An orthonormal set of higher mode functions is given by

$$
\xi_{n, j}=\frac{i^{n}}{\sqrt{n !}}\left(a_{+}^{\dagger}\right)^{n} \xi_{0, j}, \quad \bar{\xi}_{n, j}=\frac{i^{n}}{\sqrt{n !}}\left(a_{-}^{\dagger}\right)^{n} \bar{\xi}_{0, j} .
$$

Annihilation and creation operators act on these mode functions as

$$
\begin{array}{ll}
a_{+} \xi_{n, j}=i \sqrt{n} \xi_{n-1, j}, & a_{+}^{\dagger} \xi_{n, j}=-i \sqrt{n+1} \xi_{n+1, j}, \\
a_{-} \bar{\xi}_{n, j}=i \sqrt{n} \bar{\xi}_{n-1, j}, & a_{-}^{\dagger} \bar{\xi}_{n, j}=-i \sqrt{n+1} \bar{\xi}_{n+1, j} .
\end{array}
$$

The mode expansions of the fields with positive and negative charge read

$$
\begin{aligned}
& \phi^{+}=\sum_{n j} \phi_{n j}^{+} \xi_{n j}, \quad \phi^{-}=\sum_{n, j} \phi_{n, j}^{-} \bar{\xi}_{n, j}, \quad \bar{\phi}^{+}=\sum_{n, j} \bar{\phi}_{n, j}^{+} \bar{\xi}_{n, j}, \\
& \bar{\phi}^{-}=\sum_{n, j} \bar{\phi}_{n, j}^{-} \xi_{n, j}, \quad V^{+}=\sum_{n, j} V_{n, j}^{+} \xi_{n, j}, \quad V^{-}=\sum_{n, j} V_{n, j}^{-} \bar{\xi}_{n, j} .
\end{aligned}
$$

The antisymmetric tensor fields feel flux in the second and third torus. Hence, there are two sets of annihilation and creation operators, $a_{ \pm}^{2}, a_{ \pm}^{2 \dagger}$ and $a_{ \pm}^{3}, a_{ \pm}^{3 \dagger}$. Suppressing tensor indices, i.e. $W_{\gamma \delta}^{+} W_{\gamma \delta}^{-} / 2 \equiv W^{+} W^{-}$etc., and using $f_{0}^{2} \equiv \sqrt{N} f_{2}$ and $f_{0}^{3} \equiv \sqrt{N} f_{3}$, one obtains from eq. (4.4)

$$
\begin{gathered}
S_{10} \supset \int d^{10} x\left\{\int d ^ { 2 } \theta \left(\frac{1}{4} W_{0} W_{0}+\frac{1}{2} W^{+} W^{-}-i \sqrt{2 g f_{2}}\left(\phi^{1+} a_{-}^{2 \dagger} \phi^{3-}-\phi^{3+} a_{-}^{2 \dagger} \phi^{1-}\right)\right.\right. \\
\left.-i \sqrt{2 g f_{3}}\left(\phi^{2+} a_{-}^{3 \dagger} \phi^{1-}-\phi^{1+} a_{-}^{3 \dagger} \phi^{2-}\right)\right)+ \text { h.c. } \\
+\int d^{4} \theta\left(\bar{\phi}^{i+} \phi^{i+}+\bar{\phi}^{i-} \phi^{i-}+\left(2 \sqrt{N}\left(f_{2}+f_{3}\right)+\frac{2 g}{\sqrt{N}}\left(\bar{\phi}^{i+} \phi^{i+}-\bar{\phi}^{i-} \phi^{i-}\right)\right) V_{0}\right. \\
-2 i \sqrt{g}\left(\sqrt{f_{2}}\left(a_{-}^{2 \dagger} \bar{\phi}^{2+}+a_{-}^{2} \phi^{2-}\right)+\sqrt{f_{3}}\left(a_{-}^{3 \dagger} \bar{\phi}^{3+}+a_{-}^{3} \phi^{3-}\right)\right) V^{+} \\
-2 i \sqrt{g}\left(\sqrt{f_{2}}\left(a_{+}^{2 \dagger} \phi^{2+}+a_{+}^{2} \bar{\phi}^{2-}\right)+\sqrt{f_{3}}\left(a_{+}^{3 \dagger} \phi^{3+}+a_{+}^{3} \bar{\phi}^{3-}\right)\right) V^{-} \\
-2 g f_{2}\left(a_{-}^{2} V^{-} a_{+}^{2} V^{+}+a_{+}^{2 \dagger} V^{+} a_{-}^{2 \dagger} V^{-}\right) \\
\left.\left.-2 g f_{3}\left(a_{-}^{3} V^{-} a_{+}^{3} V^{+}+a_{-}^{3 \dagger} V^{-} a_{+}^{3 \dagger} V^{+}\right)\right)\right\} .
\end{gathered}
$$


The fields have a double expansion in two sets of mode functions ${ }^{6}$

$$
\begin{aligned}
& \phi^{+}\left(x ; z_{1}, z_{2}, z_{3}\right)=\sum_{n j, n^{\prime} j^{\prime}} \phi_{n j, n^{\prime} j^{\prime}}^{+}(x) \xi_{n, j}\left(z_{2}\right) \xi_{n^{\prime}, j^{\prime}}\left(z_{3}\right), \\
& \phi^{-}\left(x ; z_{1}, z_{2}, z_{3}\right)=\sum_{n j, n^{\prime} j^{\prime}} \phi_{n j, n^{\prime} j^{\prime}}^{-}(x) \bar{\xi}_{n, j}\left(z_{2}\right) \bar{\xi}_{n^{\prime}, j^{\prime}}\left(z_{3}\right), \quad \text { etc. },
\end{aligned}
$$

where, for simplicity, we only consider the lowest KK mode in the first torus. According to the quantization condition (4.1) the multiplicity in the second and third torus is two and one, respectively, giving a total multiplicity of two for all fields.

Inserting the mode expansion of the fields in the $10 \mathrm{~d}$ action, using eq. (4.7) and the orthonormality of the mode functions, and dropping the indices $j, j^{\prime}$ that label the degeneracy, one arrives at the $4 \mathrm{~d}$ effective Lagrangian

$$
\begin{aligned}
\mathcal{L}_{4} \supset \int d^{2} \theta & \frac{1}{4} W_{0} W_{0}+\sum_{n n^{\prime}}\left(\frac{1}{2} W_{n, n^{\prime}}^{+} W_{n, n^{\prime}}^{-}\right. \\
- & \sqrt{2 g f_{2}(n+1)}\left(\phi_{n+1, n^{\prime}}^{1+} \phi_{n, n^{\prime}}^{3-}-\phi_{n+1, n^{\prime}}^{3+} \phi_{n, n^{\prime}}^{1-}\right) \\
& \left.\left.-\sqrt{2 g f_{3}\left(n^{\prime}+1\right)}\left(\phi_{n, n^{\prime}+1}^{2+} \phi_{n, n^{\prime}}^{1-}-\phi_{n, n^{\prime}+1}^{1+} \phi_{n, n^{\prime}}^{2-}\right)\right)\right)+ \text { h.c. } \\
+\int d^{4} \theta & \left(2 \sqrt{N}\left(f_{2}+f_{3}\right) V_{0}+\sum_{n n^{\prime}}\left(\bar{\phi}_{n, n^{\prime}}^{i+} \phi_{n, n^{\prime}}^{i+}+\bar{\phi}_{n, n^{\prime}}^{i-} \phi_{n, n^{\prime}}^{i-}\right.\right. \\
+ & \frac{2 g}{\sqrt{N}}\left(\bar{\phi}_{n, n^{\prime}}^{i+} \phi_{n, n^{\prime}}^{i+}-\bar{\phi}_{n, n^{\prime}}^{i-} \phi_{n, n^{\prime}}^{i-}\right) V_{0} \\
- & 2\left(\left(\sqrt{g f_{2}}\left(\sqrt{n} \bar{\phi}_{n-1, n^{\prime}}^{2+}-\sqrt{n+1} \phi_{n+1, n^{\prime}}^{2-}\right)\right.\right. \\
& \left.\left.+\sqrt{g f_{3}}\left(\sqrt{n^{\prime}} \bar{\phi}_{n, n^{\prime}-1}^{3+}-\sqrt{n^{\prime}+1} \phi_{n, n^{\prime}+1}^{3-}\right)\right) V_{n, n^{\prime}}^{+}+\text {h.c. }\right) \\
+ & \left.\left.2 M_{n, n^{\prime}}^{2} V_{n, n^{\prime}}^{+} V_{n, n^{\prime}}^{-}\right)\right)
\end{aligned}
$$

where

$$
M_{n, n^{\prime}}=\left(g f_{2}(2 n+1)+g f_{3}\left(2 n^{\prime}+1\right)\right)^{1 / 2} .
$$

The magnetic flux mixes different Landau levels of the KK towers and it is therefore convenient to introduce linear combinations of the original chiral superfields,

$$
\begin{aligned}
& \phi_{n, n^{\prime}}^{-}=\frac{1}{\mu_{n, n^{\prime}}}\left(\sqrt{2 g f_{2} n} \phi_{n-1, n^{\prime}}^{3-}-\sqrt{2 g f_{3} n^{\prime}} \phi_{n, n^{\prime}-1}^{2-}\right),\left(n, n^{\prime}\right) \neq 0 ; \quad \phi_{0,0}^{-}=0, \\
& \chi_{n, n^{\prime}}^{-}=\frac{1}{\mu_{n+1, n^{\prime}+1}}\left(\sqrt{2 g f_{3}\left(n^{\prime}+1\right)} \phi_{n, n^{\prime}+1}^{3-}+\sqrt{2 g f_{2}(n+1)} \phi_{n+1, n^{\prime}}^{2-}\right)
\end{aligned}
$$

\footnotetext{
${ }^{6}$ More precisely, the fields depend on $z_{i}$ and $\bar{z}_{i}$.
} 


$$
\begin{aligned}
& \phi_{n, n^{\prime}}^{+}=\frac{1}{\mu_{n+1, n^{\prime}+1}}\left(\sqrt{2 g f_{3}\left(n^{\prime}+1\right)} \phi_{n, n^{\prime}+1}^{2+}-\sqrt{2 g f_{2}(n+1)} \phi_{n+1, n^{\prime}}^{3+}\right), \\
& \chi_{n, n^{\prime}}^{+}=\frac{1}{\mu_{n, n^{\prime}}}\left(\sqrt{2 g f_{2} n} \phi_{n-1, n^{\prime}}^{2+}+\sqrt{2 g f_{3} n^{\prime}} \phi_{n, n^{\prime}-1}^{3+}\right),\left(n, n^{\prime}\right) \neq 0 ; \quad \chi_{0,0}^{+}=0,
\end{aligned}
$$

with

$$
\mu_{n, n^{\prime}}=\left(2 g f_{2} n+2 g f_{3} n^{\prime}\right)^{1 / 2} .
$$

In terms of the new fields the $4 \mathrm{~d}$ Lagrangian reads

$$
\begin{aligned}
& \mathcal{L}_{4} \supset \int d^{2} \theta\left(\frac{1}{4} W_{0} W_{0}+\sum_{n n^{\prime}}\left(\frac{1}{2} W_{n, n^{\prime}}^{+} W_{n, n^{\prime}}^{-}-\mu_{n, n^{\prime}} \phi_{n, n^{\prime}}^{1+} \phi_{n, n^{\prime}}^{-}\right.\right. \\
& \left.\left.-\mu_{n+1, n^{\prime}+1} \phi_{n, n^{\prime}}^{1-} \phi_{n, n^{\prime}}^{+}\right)\right)+ \text {h.c. } \\
& +\int d^{4} \theta\left(2 \sqrt{N}\left(f_{2}+f_{3}\right) V_{0}+\sum_{n n^{\prime}}\left(\left|\phi_{n, n^{\prime}}^{1+}\right|^{2}+\left|\phi_{n, n^{\prime}}^{1-}\right|^{2}+\left|\phi_{n, n^{\prime}}^{+}\right|^{2}+\left|\phi_{n, n^{\prime}}^{-}\right|^{2}\right.\right. \\
& +\left|\chi_{n, n^{\prime}}^{+}\right|^{2}+\left|\chi_{n, n^{\prime}}^{-}\right|^{2}+\frac{2 g}{\sqrt{N}}\left(\left|\phi_{n, n^{\prime}}^{1+}\right|^{2}+\left|\phi_{n, n^{\prime}}^{+}\right|^{2}+\left|\chi_{n, n^{\prime}}^{+}\right|^{2}\right. \\
& \left.-\left|\phi_{n, n^{\prime}}^{1-}\right|^{2}-\left|\phi_{n, n^{\prime}}^{-}\right|^{2}-\left|\chi_{n, n^{\prime}}^{-}\right|^{2}\right) V_{0} \\
& -\sqrt{2}\left(\left(\mu_{n, n^{\prime}} \bar{\chi}_{n, n^{\prime}}^{+}-\mu_{n+1, n^{\prime}+1} \chi_{n, n^{\prime}}^{-}\right) V_{n, n^{\prime}}^{+}+\text {h.c. }\right) \\
& \left.\left.+2 M_{n, n^{\prime}}^{2} V_{n, n^{\prime}}^{+} V_{n, n^{\prime}}^{-}\right)\right)
\end{aligned}
$$

So far the diagonalization could be performed in terms of superfields. Since the magnetic flux breaks supersymmetry, one has to expand the superfields in components ${ }^{7}$ in the final step (cf. appendix C),

$$
\phi=(\phi, \psi, F), \quad V=\left(A_{\mu}, \lambda, D\right) .
$$

The mixing term between chiral and vector superfields then leads to a charged D-term and a derivative coupling between Goldstone bosons and vector fields,

$$
\begin{aligned}
& \int d^{4} \theta\left(\mu_{n, n^{\prime}} \bar{\chi}_{n, n^{\prime}}^{+}-\mu_{n+1, n^{\prime}+1} \chi_{n, n^{\prime}}^{-}\right) V_{n, n^{\prime}}^{+} \\
& =\frac{1}{2}\left(\mu_{n, n^{\prime}} \bar{\chi}_{n, n^{\prime}}^{+}-\mu_{n+1, n^{\prime}+1} \chi_{n, n^{\prime}}^{-}\right) D^{+}-\frac{i}{\sqrt{2}} M_{n, n^{\prime}} \partial_{\mu} \Pi_{n, n^{\prime}}^{-} A_{n, n^{\prime}}^{+\mu} .
\end{aligned}
$$

Here the Goldstone fields $\Pi^{-}$and the orthogonal complex scalars $\Sigma^{-}$, formed from the complex scalars $\bar{\chi}^{+}$and $\chi^{-}$, are given by

$$
\begin{aligned}
& \Pi_{n, n^{\prime}}^{-}=\frac{1}{\sqrt{2} M_{n, n^{\prime}}}\left(\mu_{n, n^{\prime}} \bar{\chi}_{n, n^{\prime}}^{+}+\mu_{n+1, n^{\prime}+1} \chi_{n, n^{\prime}}^{-}\right), \\
& \Sigma_{n, n^{\prime}}^{-}=\frac{1}{\sqrt{2} M_{n, n^{\prime}}}\left(\mu_{n+1, n^{\prime}+1} \bar{\chi}_{n, n^{\prime}}^{+}-\mu_{n, n^{\prime}} \chi_{n, n^{\prime}}^{-}\right) .
\end{aligned}
$$

\footnotetext{
${ }^{7}$ Note, that we use the same symbol for the chiral superfield and its scalar component.
} 
The vector bosons of the tower of Landau levels acquire their mass by the Stückelberg mechanism, and a shift of the vector bosons,

$$
A_{n, n^{\prime}}^{-\mu} \rightarrow A_{n, n^{\prime}}^{-\mu}+\frac{i}{M_{n, n^{\prime}}} \partial_{\mu} \Pi_{n, n^{\prime}}^{-},
$$

cancels the mixings with the Goldstone bosons as well as the kinetic terms of the Goldstone bosons. Finally, eliminating all F- and D-terms via their equations of motion, one obtains the bosonic mass terms

$$
\begin{aligned}
\mathcal{L}_{4}^{b} \supset-\sum_{n, n^{\prime}}( & M_{n, n^{\prime}}^{2}\left(A_{n, n^{\prime} \mu}^{+\mu} A_{n, n^{\prime}}^{-\mu}+\left|\phi_{n, n^{\prime}}^{1+}\right|^{2}+\left|\phi_{n, n^{\prime}}^{1-}\right|^{2}+\left|\Sigma_{n, n^{\prime}}^{-}\right|^{2}\right) \\
& \left.+\left(M_{n, n^{\prime}}^{2}-2 g f_{2}-2 g f_{3}\right)\left|\phi_{n, n^{\prime}}^{-}\right|^{2}+\left(M_{n, n^{\prime}}^{2}+2 g f_{2}+2 g f_{3}\right)\left|\phi_{n, n^{\prime}}^{+}\right|^{2}\right),
\end{aligned}
$$

where it is important to remember that $\phi_{0,0}^{-}=\Sigma_{0,0}^{-}=0$.

Consider first the lowest lying scalars,

$$
\mathcal{L}_{4}^{b} \supset-g\left(f_{2}+f_{3}\right)\left(\left|\phi_{0,0}^{1+}\right|^{2}+\left|\phi_{0,0}^{1-}\right|^{2}\right)-g\left(-f_{2}+f_{3}\right)\left|\phi_{0,1}^{-}\right|^{2}-g\left(f_{2}-f_{3}\right)\left|\phi_{1,0}^{-}\right|^{2} .
$$

These masses are in agreement with the ones given in table 3 for the $a a^{\prime}$-sector. The comparison with the string formula (3.19) is more subtle. The mass spectrum of $\phi^{1 \pm}$ corresponds to $M_{a a^{\prime}}^{2}\left(0, n, n^{\prime} ; \pm, 0,0\right)$. Since $\phi_{0,0}^{-}=0$, one can write

$$
\begin{aligned}
\sum_{n, n^{\prime}}( & \left.M_{n, n^{\prime}}^{2}-2 g f_{2}-2 g f_{3}\right)\left|\phi_{n, n^{\prime}}^{-}\right|^{2}= \\
& \sum_{n, n^{\prime}}\left(\left(M_{n, n^{\prime}}^{2}-2 g f_{2}\right)\left|\phi_{n, n^{\prime}}^{-}\right|^{2}+\left(M_{n, n^{\prime}}^{2}-2 g f_{3}\right)\left|\phi_{n, n^{\prime}}^{-}\right|^{2}-M_{n, n^{\prime}}^{2}\left|\phi_{n, n^{\prime}}^{-}\right|^{2}\right) .
\end{aligned}
$$

Hence, the spectrum of $\phi^{-}$together with one polarization state of the vector corresponds to the spectrum $M_{a a^{\prime}}^{2}\left(0, n, n^{\prime} ; 0,-, 0\right)$ together with $M_{a a^{\prime}}^{2}\left(0, n, n^{\prime} ; 0,0,-\right)$. Analogously, the spectra of $\Sigma^{-}$and $\phi^{+}$correspond to $M_{a a^{\prime}}^{2}\left(0, n, n^{\prime} ; 0,+, 0\right)$ together with $M_{a a^{\prime}}^{2}\left(0, n, n^{\prime} ; 0,0,+\right)$. Since in the string formula (3.19) massive vectors are only counted with two polarization states, the entire spectra of eqs. (3.19) and (4.26) agree. However, there is no direct correspondence for individual Landau levels.

Denoting the Weyl fermions contained in the superfields $\phi^{1 \pm}, \phi^{ \pm}, \chi^{ \pm}$and $V^{+}=V^{-\dagger}$ by $\psi^{1 \pm}, \psi^{ \pm}, \omega^{ \pm}$and $\lambda^{ \pm}$, respectively, one finds for the fermionic mass terms of the $4 \mathrm{~d}$ Lagrangian (4.20) (cf. appendix C),

$$
\begin{aligned}
\mathcal{L}_{4} \supset \sum_{n n^{\prime}}( & \mu_{n, n^{\prime}}\left(\psi_{n, n^{\prime}}^{1+} \psi_{n, n^{\prime}}^{-}+i \omega_{n, n^{\prime}}^{+} \lambda_{n, n^{\prime}}^{-}\right) \\
& \left.+\mu_{n+1, n^{\prime}+1}\left(\psi_{n, n^{\prime}}^{1-} \psi_{n, n^{\prime}}^{+}+i \omega_{n, n^{\prime}}^{-} \lambda_{n, n^{\prime}}^{+}\right)\right)+ \text {h.c. }
\end{aligned}
$$

Note that by definition, $\psi_{0,0}^{-}=\omega_{0,0}^{+}=0$ (cf. eqs. (4.15), (4.18)). Clearly, the spectrum contains two zero-modes, $\psi_{0,0}^{1+}$ and $\lambda_{0,0}^{-}$.

The structure of the $b c$-sector is identical to the one in the $a a^{\prime}$-sector. Also in the $b c$-sector the flux vanishes in the first torus, see table 1 , and only the flux densities $f_{a}^{i}$, $i=2,3$, have to be replaced by $f_{b}^{i}$ and $f_{c}^{i}$, which corresponds to a redefinition of $f_{2}$ and $f_{3}$ in eq. (4.2). 


\subsection{Chiral matter (ab-sector)}

We now turn to the chiral 'matter sector' and consider the vector and chiral superfields $V_{\alpha}^{-0}, \phi_{\alpha}^{-0} \sim N_{-1,0}$ and $V_{\alpha}^{+0}, \phi_{\alpha}^{+0} \sim \bar{N}_{1,0}$. For simplicity, we drop the superscripts " 0 " referring to zero $H_{2}$ charge in the following. The commutators of the corresponding $\mathrm{SO}(32)$ matrices are given in eq. (B.3),

$$
\left[H_{0}, T_{\alpha}^{\mp 0}\right]= \pm \frac{1}{\sqrt{N}} T_{\alpha}^{\mp 0}, \quad\left[H_{1}, T_{\alpha}^{\mp 0}\right]=\mp T_{\alpha}^{\mp 0}, \quad\left[T_{\alpha}^{-0}, T_{\beta}^{+0}\right]=\delta_{\alpha \beta}\left(\frac{1}{\sqrt{N}} H_{0}-H_{1}\right)+\ldots
$$

For anti-chiral superfields signs are exchanged. According to table 1, the flux densities $f_{1}^{i}$ in the three tori satisfy the quantization conditions $\left(\rho_{i}=L_{i}^{\prime} / L_{i}\right)$

$$
\begin{aligned}
\frac{g}{2 \pi} \int_{T_{1}^{2}} f_{1}^{1} & =\frac{2 \pi \alpha^{\prime}}{\rho_{1}} g f_{1}^{1}=1, \quad \frac{g}{2 \pi} \int_{T_{2}^{2}} f_{1}^{2}=\frac{2 \pi \alpha^{\prime}}{\rho_{2}} g f_{1}^{2}=l, \\
\frac{g}{2 \pi} \int_{T^{2}} f_{1}^{3} & =\frac{2 \pi \alpha^{\prime}}{\rho_{3}} g f_{1}^{3}=-2 .
\end{aligned}
$$

Combining the $H_{1}$ flux densities with the $H_{0}$ flux densities given in eq. (4.1), one obtains for the total flux densities, i.e. the differences between $f_{0}^{i} / \sqrt{N}$ and $f_{1}^{i}$, in the three tori

$$
\begin{aligned}
-g f_{1}^{1} & =-\frac{\rho_{1}}{2 \pi \alpha^{\prime}} \equiv-2 g f_{1}, \quad \frac{g}{\sqrt{N}} f_{0}^{2}-g f_{1}^{2}=\frac{(2-l) \rho_{2}}{2 \pi \alpha^{\prime}} \equiv-2 g f_{2}, \\
\frac{g}{\sqrt{N}} f_{0}^{3}-g f_{1}^{3} & =\frac{3 \rho_{3}}{2 \pi \alpha^{\prime}} \equiv 2 g f_{3} .
\end{aligned}
$$

Note that the flux parameters $f_{i}$ are all positive.

Using eqs. (3.11), (3.12) and (4.30), one obtains from the action (3.9) the relevant terms for the generation of boson and fermion masses,

$$
\begin{aligned}
\mathcal{L}_{10} \supset \int d^{2} \theta & \left(\frac{1}{4} W_{0} W_{0}+\frac{1}{4} W_{1} W_{1}+\frac{1}{2} W_{\alpha}^{+} W_{\alpha}^{-}\right. \\
& \left.+\varepsilon_{i j k} \phi_{\alpha}^{i-}\left(\partial_{j}-\frac{g}{\sqrt{2 N}} \chi_{0}^{j}+\frac{g}{\sqrt{2}} \chi_{1}^{j}\right) \phi_{\alpha}^{k+}\right)+ \text { h.c. } \\
+\int d^{4} \theta & \left(\bar{\chi}_{0}^{i} \chi_{0}^{i}+\bar{\chi}_{1}^{i} \chi_{1}^{i}+\bar{\phi}_{\alpha}^{i-} \phi_{\alpha}^{i-}+\bar{\phi}_{\alpha}^{i+} \phi_{\alpha}^{i+}\right. \\
& +\sqrt{2}\left(\partial_{i} \bar{\chi}_{0}^{i}+\bar{\partial}_{i} \chi_{0}^{i}\right) V_{0}+\sqrt{2}\left(\partial_{i} \bar{\chi}_{1}^{i}+\bar{\partial}_{i} \chi_{1}^{i}\right) V_{1} \\
& +\frac{g}{\sqrt{N}}\left(\bar{\phi}_{\alpha}^{i-} \phi_{\alpha}^{i-}-\bar{\phi}_{\alpha}^{i+} \phi_{\alpha}^{i+}\right) V_{0}-g\left(\bar{\phi}_{\alpha}^{i-} \phi_{\alpha}^{i-}-\bar{\phi}_{\alpha}^{i+} \phi_{\alpha}^{i+}\right) V_{1} \\
& +\sqrt{2}\left(\left(\partial_{i}-\frac{g}{\sqrt{2 N}} \chi_{0}^{i}+\frac{g}{\sqrt{2}} \chi_{1}^{i}\right) \bar{\phi}_{\alpha}^{i-}+\left(\bar{\partial}_{i}+\frac{g}{\sqrt{2 N}} \bar{\chi}_{0}^{i}-\frac{g}{\sqrt{2}} \bar{\chi}_{1}^{i}\right) \phi_{\alpha}^{i+}\right) V_{\alpha}^{-} \\
& +\sqrt{2}\left(\left(\partial_{i}+\frac{g}{\sqrt{2 N}} \chi_{0}^{i}-\frac{g}{\sqrt{2}} \chi_{1}^{i}\right) \bar{\phi}_{\alpha}^{i+}+\left(\bar{\partial}_{i}-\frac{g}{\sqrt{2 N}} \bar{\chi}_{0}^{i}+\frac{g}{\sqrt{2}} \bar{\chi}_{1}^{i}\right) \phi_{\alpha}^{i-}\right) V_{\alpha}^{+}
\end{aligned}
$$




$$
\begin{aligned}
& +\left(\bar{\partial}_{i}+\frac{g}{\sqrt{2 N}} \bar{\chi}_{0}^{i}-\frac{g}{\sqrt{2}} \bar{\chi}_{1}^{i}\right) V_{\alpha}^{+}\left(\partial_{i}+\frac{g}{\sqrt{2 N}} \chi_{0}^{i}-\frac{g}{\sqrt{2}} \chi_{1}^{i}\right) V_{\alpha}^{-} \\
& \left.+\left(\bar{\partial}_{i}-\frac{g}{\sqrt{2 N}} \bar{\chi}_{0}^{i}+\frac{g}{\sqrt{2}} \bar{\chi}_{1}^{i}\right) V_{\alpha}^{-}\left(\partial_{i}-\frac{g}{\sqrt{2 N}} \chi_{0}^{i}+\frac{g}{\sqrt{2}} \chi_{1}^{i}\right) V_{\alpha}^{+}\right) .
\end{aligned}
$$

Replacing the scalar fields $\chi_{0}^{i}$ and $\chi_{1}^{i}$ by the flux densities (4.1) and (4.31), respectively, one obtains covariant derivatives. Using eqs. (4.5) they can be replaced by annihilation and creation operators that now act on the coordinates of all three tori,

$$
\begin{aligned}
\mathcal{L}_{10} \supset \int d^{2} \theta\left(\frac{1}{4} W_{0} W_{0}+\frac{1}{4} W_{1} W_{1}+\frac{1}{2} W^{+} W^{-}-i \sqrt{2 g f_{1}}\left(\phi^{3-} a_{+}^{1} \phi^{2+}-\phi^{2-} a_{+}^{1} \phi^{3+}\right)\right. \\
\left.-i \sqrt{2 g f_{2}}\left(\phi^{1-} a_{+}^{2} \phi^{3+}-\phi^{3-} a_{+}^{2} \phi^{1+}\right)-i \sqrt{2 g f_{3}}\left(\phi^{2-} a_{-}^{3 \dagger} \phi^{1+}-\phi^{1-} a_{-}^{3 \dagger} \phi^{2+}\right)\right)+ \text { h.c. } \\
+\int d^{4} \theta\left(\bar{\chi}_{0}^{i} \chi_{0}^{i}+\bar{\chi}_{1}^{i} \chi_{1}^{i}+\bar{\phi}^{i+} \phi^{i+}+\bar{\phi}^{i-} \phi^{i-}+2\left(f_{0}^{2}+f_{0}^{3}-\frac{g}{\sqrt{N}}\left(\bar{\phi}^{i+} \phi^{i+}-\bar{\phi}^{i-} \phi^{i-}\right)\right) V_{0}\right. \\
+2\left(f_{1}^{1}+f_{1}^{2}+f_{1}^{3}+g\left(\bar{\phi}^{i+} \phi^{i+}-\bar{\phi}^{i-} \phi^{i-}\right)\right) V_{1} \\
-2 i\left(\left(\sqrt{g f_{1}}\left(a_{+}^{1} \bar{\phi}^{1-}+a_{+}^{1 \dagger} \phi^{1+}\right)+\sqrt{g f_{2}}\left(a_{+}^{2} \bar{\phi}^{2-}+a_{+}^{2 \dagger} \phi^{2+}\right)\right.\right. \\
\left.\left.+\sqrt{g f_{3}}\left(a_{-}^{3 \dagger} \bar{\phi}^{3-}+a_{-}^{3} \phi^{3+}\right)\right) V^{-}+\text {h.c. }\right) \\
-2 g f_{1}\left(a_{+}^{1 \dagger} V^{+} a_{-}^{1 \dagger} V^{-}+a_{-}^{1} V^{-} a_{+}^{1} V^{+}\right)-2 g f_{2}\left(a_{+}^{2 \dagger} V^{+} a_{-}^{2 \dagger} V^{-}\right. \\
\left.\left.+a_{-}^{2} V^{-} a_{+}^{2} V^{+}\right)-2 g f_{3}\left(a_{+}^{3 \dagger} V^{+} a_{-}^{3 \dagger} V^{-}+a_{-}^{3} V^{-} a_{+}^{3} V^{+}\right)\right)
\end{aligned}
$$

The fields have a triple expansion in three sets of mode functions

$$
\begin{aligned}
& \phi^{i-}\left(x ; z_{1}, z_{2}, z_{3}\right)=\sum_{n_{1} j_{1}, n_{2} j_{2}, n_{3} j_{3}} \phi_{n_{1} j_{1}, n_{2} j_{2}, n_{3} j_{3}}^{i-}(x) \xi_{n_{1} j_{1}}\left(z_{1}\right) \xi_{n_{2} j_{2}}\left(z_{2}\right) \xi_{n_{3} j_{3}}\left(z_{3}\right) \\
& \phi^{i+}\left(x ; z_{1}, z_{2}, z_{3}\right)=\sum_{n_{1} j_{1}, n_{2} j_{2}, n_{3} j_{3}} \phi_{n_{1} j_{1}, n_{2} j_{2}, n_{3} j_{3}}^{i+}(x) \bar{\xi}_{n_{1} j_{1}}\left(z_{1}\right) \bar{\xi}_{n_{2} j_{2}}\left(z_{2}\right) \bar{\xi}_{n_{3} j_{3}}\left(z_{3}\right), \quad \text { etc. }
\end{aligned}
$$

As in the discussion of antisymmetric tensor fields, one can now form linear combinations of the six chiral superfields $\phi^{i+}$ and $\phi^{i-}$ such that two new fields, $\Xi^{+}$and $\Xi^{-}$, mix with the vectorfield and the other four, $\phi^{ \pm}$and $\Phi^{ \pm}$, form pairwise superpotential mass terms. It is straightforward to verify that this is achieved in a two-step process,

$$
\begin{aligned}
& \phi_{n_{1}, n_{2}, n_{3}}^{-}=\frac{1}{\mu_{n_{1}, n_{2}}}\left(-\sqrt{2 g f_{1} n_{1}} \phi_{n_{1}-1, n_{2}, n_{3}}^{2-}+\sqrt{2 g f_{2} n_{2}} \phi_{n_{1}, n_{2}-1, n_{3}}^{1-}\right) \text {, } \\
& \left(n_{1}, n_{2}\right) \neq(0,0) ; \quad \phi_{0,0, n_{3}}^{-}=0, \\
& \phi_{n_{1}, n_{2}, n_{3}}^{+}=\frac{1}{\mu_{n_{1}+1, n_{2}+1}}\left(\sqrt{2 g f_{1}\left(n_{1}+1\right)} \phi_{n_{1}+1, n_{2}, n_{3}}^{2+}-\sqrt{2 g f_{2}\left(n_{2}+1\right)} \phi_{n_{1}, n_{2}+1, n_{3}}^{1+}\right) \text {, } \\
& \chi_{n_{1}, n_{2}, n_{3}}^{+}=\frac{1}{\mu_{n_{1}, n_{2}}}\left(\sqrt{2 g f_{1} n_{1}} \phi_{n_{1}-1, n_{2}, n_{3}}^{1+}+\sqrt{2 g f_{2} n_{2}} \phi_{n_{1}, n_{2}-1, n_{3}}^{2+}\right), \\
& \left(n_{1}, n_{2}\right) \neq(0,0) ; \quad \chi_{0,0, n_{3}}^{+}=0
\end{aligned}
$$




$$
\chi_{n_{1}, n_{2}, n_{3}}^{-}=\frac{1}{\mu_{n_{1}+1, n_{2}+1}}\left(\sqrt{2 g f_{1}\left(n_{1}+1\right)} \phi_{n_{1}+1, n_{2}, n_{3}}^{1-}+\sqrt{2 g f_{2}\left(n_{2}+1\right)} \phi_{n_{1}, n_{2}+1, n_{3}}^{2-}\right),
$$

with $\mu_{n_{1}, n_{2}}=\left(2 g f_{1} n_{1}+2 g f_{2} n_{2}\right)^{1 / 2}$, and, as the second step,

$$
\begin{aligned}
& \Phi_{n_{1}, n_{2}, n_{3}}^{+}=\frac{1}{\mu_{n_{1}, n_{2}, n_{3}}}\left(\sqrt{2 g f_{3} n_{3}} \chi_{n_{1}, n_{2}, n_{3}-1}^{+}+\mu_{n_{1}, n_{2}} \phi_{n_{1}, n_{2}, n_{3}}^{3+}\right), \\
& \left(n_{1}, n_{2}, n_{3}\right) \neq(0,0,0), \quad \Phi_{0,0,0}^{+}=\phi_{0,0,0}^{3+}, \\
& \Phi_{n_{1}, n_{2}, n_{3}}^{-}=\frac{1}{\mu_{n_{1}+1, n_{2}+1, n_{3}+1}}\left(\sqrt{2 g f_{3}\left(n_{3}+1\right)} \chi_{n_{1}, n_{2}, n_{3}+1}^{-}+\mu_{n_{1}+1, n_{2}+1} \phi_{n_{1}, n_{2}, n_{3}}^{3-}\right), \\
& \Xi_{n_{1}, n_{2}, n_{3}}^{+}=\frac{1}{\mu_{n_{1}, n_{2}, n_{3}+1}}\left(\mu_{n_{1}, n_{2}} \chi_{n_{1}, n_{2}, n_{3}}^{+}-\sqrt{2 g f_{3}\left(n_{3}+1\right)} \phi_{n_{1}, n_{2}, n_{3}+1}^{3+}\right), \\
& \Xi_{n_{1}, n_{2}, n_{3}}^{-}=\frac{1}{\mu_{n_{1}+1, n_{2}+1, n_{3}}}\left(\mu_{n_{1}+1, n_{2}+1} \chi_{n_{1}, n_{2}, n_{3}}^{-}-\sqrt{2 g f_{3} n_{3}} \phi_{n_{1}, n_{2}, n_{3}-1}^{3-}\right),
\end{aligned}
$$

where

$$
\mu_{n_{1}, n_{2}, n_{3}}=\left(2 g f_{1} n_{1}+2 g f_{2} n_{2}+2 g f_{3} n_{3}\right)^{1 / 2} .
$$

Note, that in eq. (4.40) the field $\Phi_{0,0,0}^{+}$is determined from the requirement $\sum_{n}\left(\left|\Phi_{n}^{+}\right|^{2}+\right.$ $\left.\left|\Xi_{n}^{+}\right|^{2}\right)=\sum_{n}\left(\left|\chi_{n}^{+}\right|^{2}+\left|\phi_{n}^{3+}\right|^{2}\right)$, where $\left(n_{1}, n_{2}, n_{3}\right) \equiv n$. In terms of the new fields the $4 \mathrm{~d}$ Lagrangian reads,

$$
\begin{gathered}
\mathcal{L}_{4} \supset \int d^{2} \theta\left(\frac{1}{4} W_{0} W_{0}+\frac{1}{4} W_{1} W_{1}+\sum_{n}\left(\frac{1}{2} W_{n}^{+} W_{n}^{-}-\mu_{n_{1}, n_{2}, n_{3}} \phi_{n}^{-} \Phi_{n}^{+}\right.\right. \\
\left.\left.\quad-\mu_{n_{1}+1, n_{2}+1, n_{3}+1} \phi_{n}^{+} \Phi_{n}^{-}\right)\right)+ \text {h.c. } \\
+\int d^{4} \theta\left(2\left(f_{0}^{2}+f_{0}^{3}\right) V_{0}+2\left(f_{1}^{1}+f_{1}^{2}+f_{1}^{3}\right) V_{1}\right. \\
+\sum_{n}\left(\left|\phi_{n}^{+}\right|^{2}+\left|\phi_{n}^{-}\right|^{2}+\left|\Phi_{n}^{+}\right|^{2}+\left|\Phi_{n}^{-}\right|^{2}+\left|\Xi_{n}^{+}\right|^{2}+\left|\Xi_{n}^{-}\right|^{2}\right. \\
\quad-\frac{g}{\sqrt{N}}\left(\left|\phi_{n}^{-}\right|^{2}+\left|\Phi_{n}^{-}\right|^{2}+\left|\Xi_{n}^{-}\right|^{2}-\left|\phi_{n}^{+}\right|^{2}-\left|\Phi_{n}^{+}\right|^{2}-\left|\Xi_{n}^{+}\right|^{2}\right) V_{0} \\
+g\left(\left|\phi_{n}^{-}\right|^{2}+\left|\Phi_{n}^{-}\right|^{2}+\left|\Xi_{n}^{-}\right|^{2}-\left|\phi_{n}^{+}\right|^{2}-\left|\Phi_{n}^{+}\right|^{2}-\left|\Xi_{n}^{+}\right|^{2}\right) V_{1} \\
+\sqrt{2}\left(\left(\mu_{n_{1}+1, n_{2}+1, n_{3}} \Xi_{n}^{-}-\mu_{n_{1}, n_{2}, n_{3}+1} \Xi_{n}^{+}\right) V_{n}^{-}+\text {h.c. }\right) \\
\left.\left.+2 M_{n_{1}, n_{2}, n_{3}}^{2} V_{n}^{+} V_{n}^{-}\right)\right)
\end{gathered}
$$

where

$$
M_{n_{1}, n_{2}, n_{3}}=\left(g f_{1}\left(2 n_{1}+1\right)+g f_{2}\left(2 n_{2}+1\right)+g f_{3}\left(2 n_{3}+1\right)\right)^{1 / 2} .
$$

At this step, supersymmetry breaking by the flux induced D-terms has to be taken into account, and vector and scalar masses have to be calculated by eliminating all auxiliary 
F- and D-terms. The mixing between $\Xi_{n}^{+}$and $\bar{\Xi}_{n}^{-}$yields the Goldstone fields $\Pi_{n}^{+}$and the orthogonal complex scalars $\Sigma_{n}^{+}$,

$$
\begin{aligned}
& \Pi_{n}^{+}=\frac{1}{\sqrt{2} M_{n_{1}, n_{2}, n_{3}}}\left(\mu_{n_{1}+1, n_{2}+1, n_{3}} \Xi_{n}^{-}+\mu_{n_{1}, n_{2}, n_{3}+1} \Xi_{n}^{+}\right), \\
& \Sigma_{n}^{+}=\frac{1}{\sqrt{2} M_{n_{1}, n_{2}, n_{3}}}\left(-\mu_{n_{1}, n_{2}, n_{3}+1} \bar{\Xi}_{n}^{-}+\mu_{n_{1}+1, n_{2}+1, n_{3}} \Xi_{n}^{+}\right) .
\end{aligned}
$$

The vector bosons of the tower of Landau levels acquire their mass by the Stückelberg mechanism, and a shift of the vector bosons,

$$
A_{n}^{+\mu} \rightarrow A_{n}^{+\mu}-\frac{i}{M_{n_{1}, n_{2}, n_{3}}} \partial_{\mu} \Pi_{n}^{+},
$$

cancels the mixings with the Goldstone bosons as well as the kinetic terms of the Goldstone bosons. The final result for the bosonic part of the $4 \mathrm{~d}$ Lagrangian (4.45) reads

$$
\begin{aligned}
\mathcal{L}_{4}^{b} \supset-\sum_{n}( & M_{n_{1}, n_{2}, n_{3}}^{2}\left(A_{n}^{+\mu} A_{n}^{-\mu}+\left|\Sigma_{n}^{+}\right|^{2}\right) \\
& +\left(M_{n_{1}, n_{2}, n_{3}}^{2}-2 g f_{1}-2 g f_{2}\right)\left|\phi_{n}^{-}\right|^{2}+\left(M_{n_{1}, n_{2}, n_{3}}^{2}+2 g f_{1}+2 g f_{2}\right)\left|\phi_{n}^{+}\right|^{2} \\
& \left.+\left(M_{n_{1}, n_{2}, n_{3}}^{2}+2 g f_{3}\right)\left|\Phi_{n}^{-}\right|^{2}+\left(M_{n_{1}, n_{2}, n_{3}}^{2}-2 g f_{3}\right)\left|\Phi_{n}^{+}\right|^{2}\right)
\end{aligned}
$$

where, by definition, $\phi_{0,0, n_{3}}^{-}=0$ and $\Phi_{0,0,0}^{+}=\phi_{0,0,0}^{3+}$ (see eqs. (4.36), (4.40)). The scalars with smallest masses are $\phi_{0,1,0}^{-}, \phi_{1,0,0}^{-}$and $\Phi_{0,0,0}^{+}$,

$$
\begin{aligned}
\mathcal{L}_{4}^{b} \supset- & g\left(-f_{1}+f_{2}+f_{3}\right)\left|\phi_{0,1,0}^{-}\right|^{2}-g\left(f_{1}-f_{2}+f_{3}\right)\left|\phi_{1,0,0}^{-}\right|^{2} \\
& -g\left(f_{1}+f_{2}-f_{3}\right)\left|\Phi_{0,0,0}^{+}\right|^{2} .
\end{aligned}
$$

These masses are in agreement with the ones given in table 3 for the $a b$-sector.

Denoting the Weyl fermions contained in the superfields $\phi^{ \pm}, \Phi^{ \pm}, \Xi^{ \pm}$and $V^{+}=V^{-\dagger}$ by $\psi^{ \pm}, \psi^{\prime \pm}, \omega^{ \pm}$and $\lambda^{ \pm}$, respectively, one finds for the fermionic mass terms of the $4 \mathrm{~d}$ Lagrangian (4.45) (cf. appendix C),

$$
\begin{aligned}
\mathcal{L}_{4} \supset \sum_{n} & \left(\mu_{n_{1}, n_{2}, n_{3}} \psi_{n}^{-} \psi_{n}^{\prime+}+i \mu_{n_{1}, n_{2}, n_{3}+1} \omega_{n}^{+} \lambda_{n}^{-}\right. \\
& \left.+\mu_{n_{1}+1, n_{2}+1, n_{3}+1} \psi_{n}^{+} \psi_{n}^{\prime-}+i \mu_{n_{1}+1, n_{2}+1, n_{3}} \omega_{n}^{-} \lambda_{n}^{+}\right)+ \text {h.c. }
\end{aligned}
$$

Note, that by definition, $\psi_{0,0, n_{3}}^{-}=0$. Hence, the spectrum contains one zero-mode, $\psi_{0,0,0}^{\prime+} \subset \Phi_{0,0,0}^{+}$.

The number of flux quanta in the first, second and third torus is $1, l-2$ and 3 , respectively. All fields therefore have a multiplicity of $3(l-2)$, in agreement with the intersection number for the $a b$-sector listed in table 2 . The multiplicity of fields is labeled by the indices $j_{1}, j_{2}, j_{3}$. The quadratic part of the $4 \mathrm{~d}$ Lagrangian, given in eqs. (4.50) and (4.52), is diagonal and the same for all fields. However, due to the non-trivial profile of the mode functions in the compact space, Yukawa couplings depend on $j_{1}, j_{2}, j_{3}$. 


\section{Higgs sector}

In the $b c$ - and $b c^{\prime}$-sectors of the D-brane model there are no chiral fermions, and both sectors contain a tachyon, see table 3 . In this section we will analyze the $b c^{\prime}$-sector in detail. According to table 1, brane $b$ and brane $c^{\prime}$ are parallel in two tori, their distances being moduli. In the T-dual flux compactification these moduli correspond to Wilson lines. From table 1 and eq. (3.17) one obtains for the background fields in the three tori,

$$
\begin{aligned}
& g f_{1}^{1}=\frac{\rho_{1}}{2 \pi \alpha^{\prime}} \bar{z}_{1}, \quad g \chi_{1}^{2}=\frac{l \rho_{2}}{2 \pi \alpha^{\prime}} \frac{\bar{z}_{2}}{\sqrt{2}}+g \xi_{1}^{2}, \quad g \chi_{1}^{3}=-\frac{2 \rho_{3}}{2 \pi \alpha^{\prime}} \frac{\bar{z}_{3}}{\sqrt{2}}+g \xi_{1}^{3}, \\
& g f_{2}^{1}=\frac{\rho_{1}}{2 \pi \alpha^{\prime}} \bar{z}_{1}, \quad g \chi_{2}^{2}=-\frac{l \rho_{2}}{2 \pi \alpha^{\prime}} \frac{\bar{z}_{2}}{\sqrt{2}+g \xi_{2}^{2},} \quad g \chi_{2}^{3}=\frac{\rho_{3}}{2 \pi \alpha^{\prime}} \frac{\bar{z}_{3}}{\sqrt{2}}+g \xi_{2}^{3} .
\end{aligned}
$$

The $b c^{\prime}$-sector contains the vector and chiral superfields $V^{++}, \phi^{i++}, V^{--}$and $\phi^{i--}$. The charges with respect to $H_{1}$ and $H_{2}$ are identical. For notational simplicity, we shall drop one of the superscripts in the following. The commutators of the relevant $\mathrm{SO}(32)$ matrices are given in eq. (B.9),

$$
\left[H_{1}, X^{ \pm \pm}\right]= \pm X^{ \pm \pm}, \quad\left[H_{2}, X^{ \pm \pm}\right]= \pm X^{ \pm \pm}, \quad\left[X^{++}, X^{--}\right]=H_{1}+H_{2} .
$$

Combining the $H_{1}$ and $H_{2}$ background fields in eq. (5.1), one obtains for the total flux densities and Wilson lines in the three tori

$$
\begin{aligned}
& g\left(f_{1}^{1}+f_{2}^{1}\right)=\frac{\rho_{1}}{\pi \alpha^{\prime}} \bar{z}_{1} \equiv 4 g f \bar{z}_{1}, \quad g\left(\chi_{1}^{2}+\chi_{2}^{2}\right)=g\left(\xi_{1}^{2}+\xi_{2}^{2}\right) \equiv g \sqrt{2} \xi_{2}, \\
& g\left(\chi_{1}^{3}+\chi_{2}^{3}\right)=g\left(\xi_{1}^{3}+\xi_{2}^{3}\right) \equiv g \sqrt{2} \xi_{3} .
\end{aligned}
$$

Using eqs. (3.9), (3.11), (3.12) and (B.9), and inserting the background fields (5.2), one obtains for the quadratic part of the 10d Lagrangian,

$$
\begin{aligned}
\mathcal{L}_{10} \supset \int d^{2} \theta( & \frac{1}{4} W_{1} W_{1}+\frac{1}{4} W_{2} W_{2}+\frac{1}{2} W^{+} W^{-} \\
+ & \phi^{3+}\left(\partial_{1}-2 g f \bar{z}_{1}\right) \phi^{2-}-\phi^{2+}\left(\partial_{1}-2 g f \bar{z}_{1}\right) \phi^{3-} \\
+ & \phi^{1+}\left(\partial_{2}-g \xi_{2}\right) \phi^{3-}-\phi^{3+}\left(\partial_{2}-g \xi_{2}\right) \phi^{1-} \\
& \left.+\phi^{2+}\left(\partial_{3}-g \xi_{3}\right) \phi^{1-}-\phi^{1+}\left(\partial_{3}-g \xi_{3}\right) \phi^{2-}\right)+ \text { h.c. } \\
+\int d^{4} \theta & \left(\bar{\phi}^{i+} \phi^{i+}+\bar{\phi}^{i-} \phi^{i-}+4 f\left(V_{1}+V_{2}\right)+g\left(\bar{\phi}^{i+} \phi^{i+}-\bar{\phi}^{i-} \phi^{i-}\right)\left(V_{1}+V_{2}\right)\right. \\
& +\sqrt{2}\left(\left(\left(\partial_{1}+2 g f \bar{z}_{1}\right) \bar{\phi}^{1-}+\left(\partial_{2}+g \xi_{2}\right) \bar{\phi}^{2-}+\left(\partial_{3}+g \xi_{3}\right) \bar{\phi}^{3-}\right.\right. \\
& \left.\left.+\left(\bar{\partial}_{1}-2 g f z_{1}\right) \phi^{1+}+\left(\bar{\partial}_{2}-g \bar{\xi}_{2}\right) \phi^{2+}+\left(\bar{\partial}_{3}-g \bar{\xi}_{3}\right) \phi^{3+}\right) V^{-}+\text {h.c. }\right) \\
& +\left(\bar{\partial}_{1}-2 g f z_{1}\right) V^{+}\left(\partial_{1}-2 g f \bar{z}_{1}\right) V^{-}+\left(\bar{\partial}_{2}-g \bar{\xi}_{2}\right) V^{+}\left(\partial_{2}-g \xi_{2}\right) V^{-} \\
& +\left(\bar{\partial}_{3}-g \bar{\xi}_{3}\right) V^{+}\left(\partial_{3}-g \xi_{3}\right) V^{-}+\left(\bar{\partial}_{1}+2 g f z_{1}\right) V^{-}\left(\partial_{1}+2 g f \bar{z}_{1}\right) V^{+} \\
& \left.+\left(\bar{\partial}_{2}+g \bar{\xi}_{2}\right) V^{-}\left(\partial_{2}+g \xi_{2}\right) V^{+}+\left(\bar{\partial}_{3}+g \bar{\xi}_{3}\right) V^{+}\left(\partial_{3}+g \xi_{3}\right) V^{-}\right) .
\end{aligned}
$$


The fields feel magnetic flux only in the first torus. Hence the mode functions are harmonic oscillator wave functions in the first torus and ordinary KK mode functions in the second and third torus,

$$
\begin{aligned}
\phi^{i+}\left(x ; z_{1}, z_{2}, z_{3}\right) & =\sum_{n j, m l, m^{\prime} l^{\prime}} \phi_{n j, m l, m^{\prime} l^{\prime}}^{i+}(x) \xi_{n j}\left(z_{1}\right) \bar{\eta}_{m l}\left(z_{2}\right) \bar{\eta}_{m^{\prime} l^{\prime}}\left(z_{3}\right), \\
\phi^{i-}\left(x ; z_{1}, z_{2}, z_{3}\right) & =\sum_{n j, m l, m^{\prime} l^{\prime}} \phi_{n j, m l, m^{\prime} l^{\prime}}^{i-}(x) \bar{\xi}_{n j}\left(z_{1}\right) \eta_{m l}\left(z_{2}\right) \eta_{m^{\prime} l^{\prime}}\left(z_{3}\right), \quad \text { etc. },
\end{aligned}
$$

where

$$
\eta_{l m}(z)=e^{z \mu_{l m}-\overline{z \mu}_{l m}}=\bar{\eta}_{-l-m}, \quad \mu_{l m}=2 \pi(m+i l) \equiv \mu_{\eta} .
$$

Replacing covariant derivatives with flux by annihilation and creation operators according to eq. (4.5), inserting the mode expansion (5.4) for the second and third torus and keeping for the two $\mathrm{U}(1)$ factors only the lowest mode, one arrives at

$$
\begin{aligned}
& \mathcal{L}_{10} \supset \int d^{2} \theta\left(\frac{1}{4} W_{1} W_{1}+\frac{1}{4} W_{2} W_{2}+\sum_{\eta \eta^{\prime}}\left(\frac{1}{2} W_{\overline{\eta \eta^{\prime}}}^{+} W_{\eta \eta^{\prime}}^{-}\right.\right. \\
& -i \sqrt{4 g f}\left(\phi_{\overline{\eta \eta^{\prime}}}^{3+} a_{-}^{\dagger} \phi_{\eta \eta^{\prime}}^{2-}-\phi_{\overline{\eta \eta^{\prime}}}^{2+} a_{-}^{\dagger} \phi_{\eta \eta^{\prime}}^{3-}\right)+\phi_{\overline{\eta \eta^{\prime}}}^{1+} M_{\eta} \phi_{\eta \eta^{\prime}}^{3-} \\
& \left.\left.-\phi_{\overline{\eta \eta^{\prime}}}^{3+} M_{\eta} \phi_{\eta \eta^{\prime}}^{1-}+\phi_{\overline{\eta \eta^{\prime}}}^{2+} M_{\eta^{\prime}} \phi_{\eta \eta^{\prime}}^{1-}-\phi_{\overline{\eta \eta^{\prime}}}^{1+} M_{\eta^{\prime}} \phi_{\eta \eta^{\prime}}^{2--}\right)\right)+ \text { h.c. } \\
& +\int d^{4} \theta\left(4 f\left(V_{1}+V_{2}\right)+\sum_{\eta, \eta^{\prime}}\left(\bar{\phi}_{\overline{\eta \eta^{\prime}}}^{i+} \phi_{\eta \eta^{\prime}}^{i+}+\bar{\phi}_{\overline{\eta \eta^{\prime}}}^{i-} \phi_{\eta \eta^{\prime}}^{i-}\right.\right. \\
& +g\left(V_{1}+V_{2}\right)\left(\left|\phi_{\eta \eta^{\prime}}^{i+}\right|^{2}-\left|\phi_{\eta \eta^{\prime}}^{i-}\right|^{2}\right)+\sqrt{2}\left(\left(-i \sqrt{4 g f}\left(a_{+} \bar{\phi}_{\overline{\eta \eta^{\prime}}}^{1-}+a_{+}^{\dagger} \phi_{\overline{\eta \eta^{\prime}}}^{1+}\right)\right.\right. \\
& \left.\left.-M_{\eta} \bar{\phi}_{\overline{\eta \eta^{\prime}}}^{2-}-M_{\eta^{\prime}} \bar{\phi}_{\bar{\eta} \eta^{\prime}}^{3-}+\bar{M}_{\eta} \phi_{\bar{\eta} \eta^{\prime}}^{2+}+\bar{M}_{\eta^{\prime}} \phi_{\overline{\eta \eta^{\prime}}}^{3+}\right) V_{\eta \eta^{\prime}}^{-}+\text {h.c. }\right) \\
& \left.\left.-4 g f\left(a_{+}^{\dagger} V_{\bar{\eta}{\eta^{\prime}}^{\prime}}^{+} a_{-}^{\dagger} V_{\eta \eta^{\prime}}^{-}+a_{-} V_{\bar{\eta} \eta^{\prime}}^{-} a_{+} V_{\eta \eta^{\prime}}^{+}\right)+2\left(\left|M_{\eta}\right|^{2}+\left|M_{\eta^{\prime}}\right|^{2}\right) V_{\overline{\eta \eta^{\prime}}}^{+} V_{\eta \eta^{\prime}}^{-}\right)\right) \text {, }
\end{aligned}
$$

where

$$
M_{\eta}=\mu_{\eta}-g \xi_{2}, \quad M_{\eta^{\prime}}=\mu_{\eta^{\prime}}-g \xi_{3}
$$

are mass terms that depend on the Wilson lines.

Consider first the case without flux, i.e., $f=0$. In this case supersymmetry is unbroken and, for simplicity, we restrict ourselves to mode functions (5.4) that are constant in the first torus. Then one can easily diagonalize the Lagrangian. Defining the superfields ${ }^{8}$

$$
\begin{array}{ll}
\phi_{\overline{\eta \eta^{\prime}}}^{+}=\frac{1}{\left|M_{\eta \eta^{\prime}}\right|}\left(M_{\eta^{\prime}} \phi_{\overline{\eta \eta^{\prime}}}^{2+}-M_{\eta} \phi_{\overline{\eta \eta^{\prime}}}^{3+}\right), \quad \phi_{\eta \eta^{\prime}}^{-}=\frac{1}{\left|M_{\eta \eta^{\prime}}\right|}\left(M_{\eta^{\prime}} \phi_{\eta \eta^{\prime}}^{2-}-M_{\eta} \phi_{\eta \eta^{\prime}}^{3-}\right), \\
\chi_{\overline{\eta \eta^{\prime}}}^{+}=\frac{1}{\left|M_{\eta \eta^{\prime}}\right|}\left(\bar{M}_{\eta} \phi_{\overline{\eta \eta^{\prime}}}^{2+}+\bar{M}_{\eta^{\prime}} \phi_{\overline{\eta \eta^{\prime}}}^{3+}\right), \quad \chi_{\eta \eta^{\prime}}^{-}=\frac{1}{\left|M_{\eta \eta^{\prime}}\right|}\left(\bar{M}_{\eta} \phi_{\eta \eta^{\prime}}^{2-}+\bar{M}_{\eta^{\prime}} \phi_{\eta \eta^{\prime}}^{3-}\right),
\end{array}
$$

\footnotetext{
${ }^{8}$ The following discussion holds for $M_{\eta \eta^{\prime}} \neq 0$. For $M_{\eta \eta^{\prime}}=M_{\eta}=M_{\eta^{\prime}}=0$, the fields $\phi_{00}^{2 \pm}$ and $\phi_{00}^{3 \pm}$ do not mix.
} 
where $\left|M_{\eta \eta^{\prime}}\right|=\left(\left|M_{\eta}\right|^{2}+\left|M_{\eta^{\prime}}\right|^{2}\right)^{1 / 2}$, and shifting the vector superfield,

$$
V_{\eta \eta^{\prime}}^{+} \rightarrow V_{\eta \eta^{\prime}}^{+}-\frac{1}{\sqrt{2}\left|M_{\eta \eta^{\prime}}\right|}\left(\chi_{\eta \eta^{\prime}}^{+}-\bar{\chi}_{\eta \eta^{\prime}}^{-}\right)
$$

one obtains

$$
\begin{aligned}
\mathcal{L}_{10} \supset \int d^{2} \theta( & \frac{1}{4} W_{1} W_{1}+\frac{1}{4} W_{2} W_{2} \\
& \left.+\sum_{\eta \eta^{\prime}}\left(\frac{1}{2} W_{\overline{\eta \eta^{\prime}}}^{+} W_{\eta \eta^{\prime}}^{-}+\left|M_{\eta \eta^{\prime}}\right|\left(\phi_{\eta \eta^{\prime}}^{1-} \phi_{\overline{\eta \eta^{\prime}}}^{+}-\phi_{\bar{\eta} \underline{\eta}^{\prime}}^{1+} \phi_{\eta \eta^{\prime}}^{-}\right)\right)\right)+ \text {h.c. } \\
& +\int d^{4} \theta \sum_{\eta \eta^{\prime}}\left(\left|\phi_{\eta \eta^{\prime}}^{1+}\right|^{2}+\left|\phi_{\eta \eta^{\prime}}^{1-}\right|^{2}+\left|\phi_{\eta \eta^{\prime}}^{+}\right|^{2}+\left|\phi_{\eta \eta^{\prime}}^{-}\right|^{2}+2\left|M_{\eta \eta^{\prime}}\right|^{2} V_{\overline{\eta \eta^{\prime}}}^{-} V_{\eta \eta^{\prime}}^{+}\right)
\end{aligned}
$$

The Goldstone chiral multiplets $\chi_{\eta \eta^{\prime}}^{ \pm}$have been removed from the Lagrangian, and a complex vector multiplet and four chiral multiplets all have the same mass $M_{\eta \eta^{\prime}}$, corresponding to $\mathcal{N}=4$ supersymmetry.

The magnetic flux in the first torus mixes different Landau levels of $\phi_{n, \eta \eta^{\prime}}^{1 \pm}$ and $\chi_{n, \eta \eta^{\prime}}^{ \pm}$. Now it is convenient to introduce the superfields

$$
\begin{aligned}
& \Xi_{n, \overline{\eta \eta^{\prime}}}^{+}=\frac{1}{\mu_{n, \eta \eta^{\prime}}}\left(\left|M_{\eta \eta^{\prime}}\right| \chi_{n, \overline{\eta \eta^{\prime}}}^{+}-\sqrt{4 g f n} \phi_{n-1, \overline{\eta \eta^{\prime}}}^{1+}\right), \\
& \Xi_{n, \eta \eta^{\prime}}^{-}=\frac{1}{\mu_{n+1, \eta \eta^{\prime}}}\left(\left|M_{\eta \eta^{\prime}}\right| \chi_{n, \eta \eta^{\prime}}^{-}-\sqrt{4 g f(n+1)} \phi_{n+1, \eta \eta^{\prime}}^{1-}\right), \\
& \Phi_{n, \overline{\eta \eta^{\prime}}}^{+}=\frac{1}{\mu_{n+1, \eta \eta^{\prime}}}\left(\sqrt{4 g f(n+1)} \chi_{n+1, \overline{\eta \eta^{\prime}}}^{+}+\left|M_{\eta \eta^{\prime}}\right| \phi_{n, \eta \eta^{\prime}}^{1+}\right), \\
& \Phi_{n, \eta \eta^{\prime}}^{-}=\frac{1}{\mu_{n, \eta \eta^{\prime}}}\left(\sqrt{4 g f n} \chi_{n-1, \eta \eta^{\prime}}^{-}+\left|M_{\eta \eta^{\prime}}\right| \phi_{n, \eta \eta^{\prime}}^{1-}\right),
\end{aligned}
$$

with

$$
\mu_{n, \eta \eta^{\prime}}=\left(4 g f n+\left|M_{\eta \eta^{\prime}}\right|^{2}\right)^{1 / 2} .
$$

Using eqs. (4.5) and (5.6), a straightforward calculation yields for the $4 \mathrm{~d}$ Lagrangian,

$$
\begin{gathered}
\mathcal{L}_{10} \supset \int d^{2} \theta\left(\frac{1}{4} W_{1} W_{1}+\frac{1}{4} W_{2} W_{2}+\sum_{n, \eta \eta^{\prime}}\left(\frac{1}{2} W_{n, \overline{\eta \eta^{\prime}}}^{+} W_{n, \eta \eta^{\prime}}^{-}\right.\right. \\
\left.\left.+\mu_{n, \eta \eta^{\prime}} \Phi_{n, \eta \eta^{\prime}}^{-} \phi_{n, \overline{\eta \eta^{\prime}}}^{+}-\mu_{n+1, \eta \eta^{\prime}} \Phi_{n, \overline{\eta \eta^{\prime}}}^{+} \phi_{n, \eta \eta^{\prime}}^{-}\right)\right)+ \text {h.c. } \\
+\int d^{4} \theta\left(\begin{array}{l}
4 f\left(V_{1}+V_{2}\right)+\sum_{n, \eta \eta^{\prime}}\left(\left|\phi_{n, \eta \eta^{\prime}}^{+}\right|^{2}+\left|\phi_{n, \eta \eta^{\prime}}^{-}\right|^{2}+\left|\Phi_{n, \eta \eta^{\prime}}^{+}\right|^{2}+\left|\Xi_{n, \eta \eta^{\prime}}^{+}\right|^{2}\right. \\
+\left|\Phi_{n, \eta \eta^{\prime}}^{-}\right|^{2}+\left|\Xi_{n, \eta \eta^{\prime}}^{-}\right|^{2}+g\left(\left|\phi_{n, \eta \eta^{\prime}}^{+}\right|^{2}+\left|\Phi_{n, \eta \eta^{\prime}}^{+}\right|^{2}+\left|\Xi_{n, \eta \eta^{\prime}}^{+}\right|^{2}\right. \\
\left.-\left|\phi_{n, \eta \eta^{\prime}}^{-}\right|^{2}-\left|\Phi_{n, \eta \eta^{\prime}}^{-}\right|^{2}-\left|\Xi_{n, \eta \eta^{\prime}}^{-}\right|^{2}\right)\left(V_{1}+V_{2}\right)
\end{array}\right.
\end{gathered}
$$




$$
\begin{aligned}
& +\sqrt{2}\left(\left(\mu_{n, \eta \eta^{\prime}} \Xi_{n, \overline{\eta \eta^{\prime}}}^{+}-\mu_{n+1, \eta \eta^{\prime}} \Xi_{n, \overline{\eta \eta^{\prime}}}^{-}\right) V_{n \eta \eta^{\prime}}^{-}+\text {h.c. }\right) \\
& \left.\left.+2 M_{n, \eta \eta^{\prime}}^{2} V_{\overline{\eta \eta^{\prime}}}^{+} V_{\eta \eta^{\prime}}^{-}\right)\right)
\end{aligned}
$$

where

$$
M_{n, \eta \eta^{\prime}}=\left(2 g f(2 n+1)+\left|M_{\eta \eta^{\prime}}\right|^{2}\right)^{1 / 2} .
$$

Like in the previous section the Goldstone bosons giving mass to the vector bosons $A_{n, \eta \eta^{\prime}}^{+\mu}$ are identified as $^{9}$

$$
\Pi_{n, \overline{\eta \eta^{\prime}}}^{+}=\frac{1}{\sqrt{2} M_{n, \eta \eta^{\prime}}}\left(\mu_{n, \eta \eta^{\prime}} \Xi_{n, \overline{\eta \eta^{\prime}}}^{+}+\mu_{n+1, \eta \eta^{\prime}} \bar{\Xi}_{n, \overline{\eta \eta^{\prime}}}^{-}\right)
$$

with the orthogonal complex scalars

$$
\Sigma_{n, \overline{\eta \eta^{\prime}}}^{+}=\frac{1}{\sqrt{2} M_{n, \eta \eta^{\prime}}}\left(\mu_{n, \eta \eta^{\prime}} \Xi_{n, \overline{\eta \eta^{\prime}}}^{+}-\mu_{n+1, \eta \eta^{\prime}} \bar{\Xi}_{n, \overline{\eta \eta^{\prime}}}^{-}\right)
$$

where we have used $\mu_{n+1, \eta \eta^{\prime}}^{2}+\mu_{n, \eta \eta^{\prime}}^{2}=2 M_{n, \eta \eta^{\prime}}^{2}$. The kinetic terms of the tower of Goldstone bosons are removed by shifting the vector bosons,

$$
A_{n, \eta \eta^{\prime}}^{+\mu} \rightarrow A_{n, \eta \eta^{\prime}}^{+\mu}+\frac{i}{M_{n, \eta \eta^{\prime}}} \partial^{\mu} \Pi_{n, \eta \eta^{\prime}}^{+} .
$$

Eliminating all F-terms and the D-terms $D_{1}, D_{2}, D_{ \pm}$by their equations of motion, one obtains for the bosonic mass terms

$$
\begin{gathered}
\mathcal{L}_{4}^{b} \supset-\sum_{n, \eta \eta^{\prime}}\left(M_{n, \eta \eta^{\prime}}^{2}\left(A_{n, \eta \eta^{\prime} \mu}^{+} A_{n, \eta \eta^{\prime}}^{-\mu}+\left|\phi_{n, \eta \eta^{\prime}}^{+}\right|^{2}+\left|\phi_{n, \eta \eta^{\prime}}^{-}\right|^{2}+\left|\Sigma_{n, \eta \eta^{\prime}}^{-}\right|^{2}\right)\right. \\
\left.+\left(M_{n, \eta \eta^{\prime}}^{2}-4 g f\right)\left|\Phi_{n, \eta \eta^{\prime}}^{-}\right|^{2}+\left(M_{n, \eta \eta^{\prime}}^{2}+4 g f\right)\left|\Phi_{n, \eta \eta^{\prime}}^{+}\right|^{2}\right) .
\end{gathered}
$$

Note that the mass of $\Phi_{0,00}^{-}$,

$$
M^{2}\left[\Phi_{0,00}^{-}\right]=-2 g f+g^{2}\left(\left|\xi_{2}\right|^{2}+\left|\xi_{3}\right|^{2}\right),
$$

is tachyonic for $\left|\xi_{2}\right|^{2}+\left|\xi_{3}\right|^{2}<f / g$. The implications will be studied in the subsequent section. The boson masses are consistent with the string formula (3.19) for the internal helicities $(0,0,0),( \pm, 0,0),(0, \pm, 0),(0,0, \pm)$.

Denoting the Weyl fermions contained in the superfields $\phi^{ \pm}, \Phi^{ \pm}, \Xi^{ \pm}$and $V^{+}=V^{-\dagger}$ by $\psi^{ \pm}, \psi^{\prime \pm}, \omega^{ \pm}$and $\lambda^{ \pm}$, respectively, one obtains for the fermion mass terms (see eq. (5.12)),

$$
\begin{aligned}
\mathcal{L}_{4}^{f} \supset-( & \mu_{n, \eta \eta^{\prime}}\left(\psi_{n, \overline{\eta \eta^{\prime}}}^{-} \psi_{n, \overline{\eta \eta^{\prime}}}^{+}-i \omega_{n, \overline{\eta \eta^{\prime}}}^{-} \lambda_{n, \overline{\eta \eta^{\prime}}}^{+}\right) \\
& \left.+\mu_{n+1, \eta \eta^{\prime}}\left(\psi_{n, \overline{\eta \eta^{\prime}}}^{+} \psi_{n, \overline{\eta \eta^{\prime}}}^{\prime-}-i \omega_{n, \overline{\eta \eta^{\prime}}}^{+} \lambda_{n, \overline{\eta \eta^{\prime}}}^{-}\right)\right)+ \text {h.c. }
\end{aligned}
$$

For vanishing Wilson lines there are four vector-like zero modes, $\psi_{0, \overline{\eta \eta^{\prime}}}^{-}, \psi_{0, \overline{\eta \eta^{\prime}}}^{\prime+}, \omega_{0, \overline{\eta \eta^{\prime}}}^{-}$ and $\lambda_{0, \overline{\eta \eta}}^{+}$. In the string formula (3.19) the mass spectrum is obtained for the helicities $(-1 / 2, \pm 1 / 2, \pm 1 / 2)$ and $(1 / 2, \pm 1 / 2, \pm 1 / 2)$. There are two flux quanta in the first torus, hence the multiplicity of all fields is two. In the case $M_{n, \eta \eta^{\prime}}=0$, corresponding to a compactification from six dimensions to four dimensions, the mass spectrum has previously been obtained in [22].

\footnotetext{
${ }^{9}$ Note that we use the same notation for a chiral superfield and its scalar component.
} 


\section{$5.1 b c$-sector}

The $b c$-sector is very similar to the $a a^{\prime}$-sector. In both cases the magnetic flux is non-zero only in the second and third torus. We therefore do not treat this case in detail but only mention some key features which are relevant for the discussion of tachyon condensation in section 7 .

The sector contains the vector and chiral superfields $V^{+-}, \phi^{i+-}, V^{-+}$and $\phi^{i-+}$, i.e., the charges with respect to $H_{1}$ and $H_{2}$ are opposite. The commutators of the relevant $\mathrm{SO}(32)$ matrices read (see eq. (B.8)),

$$
\left[H_{1}, T^{ \pm \mp}\right]= \pm T^{ \pm \mp}, \quad\left[H_{2}, T^{ \pm \mp}\right]=\mp T^{ \pm \mp}, \quad\left[T^{+-}, T^{-+}\right]=H_{1}-H_{2} .
$$

For zero Wilson lines, one obtains for the background fields given in eq. (5.1) the total flux densities in the three tori

$$
\begin{array}{ll}
g\left(f_{1}^{1}-f_{2}^{1}\right) & =0, \\
g\left(f_{1}^{3}-f_{2}^{3}\right) & =-3 \frac{\rho_{3}}{2 \pi \alpha^{\prime}} \bar{z}_{3} \equiv-g f_{3} \bar{z}_{3} .
\end{array}
$$

The crucial difference compared to the $a a^{\prime}$-sector is the opposite sign of the flux densities in the second and third torus. In the derivation of the effective $4 \mathrm{~d}$ action this exchanges annihilation and creation operators in various steps of the calculation. Taking this into account, all relevant $F$ - and $D$-terms can be essentially read off from eq. (4.13).

\section{$6 \quad$ Effective potential}

We are now ready to calculate the one-loop effective potential from the effective field theory. We start with the potential for Wilson lines in the $b c^{\prime}$-sector, then we discuss the potential in the $a b$-sector which is independent of Wilson lines and depends only on volume moduli. We shall perform the calculation for the effective field theory discussed in the previous section, summing over the full towers of Landau levels and KK modes, and we shall then compare the result with a string calculation.

\subsection{Field theory calculation}

The one-loop effective potential is given by the well known Coleman-Weinberg expression

$$
V(\xi)=\frac{1}{2} \sum_{I}(-)^{F} \int \frac{d^{4} k}{(2 \pi)^{4}} \ln \left(k^{2}+M_{I}^{2}(\xi)\right),
$$

where the sum extends over all bosonic and fermionic states. The masses in the $b c^{\prime}$-sector are denoted by $M_{I}(\xi), F$ denotes fermion number, $I$ accounts for Landau levels and KK quantum numbers, and $\xi$ represents real and imaginary parts of the Wilson lines in the second and third torus, i.e. $\xi=\left(\xi_{1}, \xi_{2}, \xi_{3}, \xi_{4}\right)=\left(\operatorname{Re} \xi_{2}, \operatorname{Im} \xi_{2}, \operatorname{Re} \xi_{3}, \operatorname{Im} \xi_{3}\right)$. Using the Schwinger representation of propagators one has

$$
\int \frac{d^{4} k}{(2 \pi)^{4}} \ln \left(k^{2}+M_{I}^{2}\right)=-\frac{1}{16 \pi^{2}} \int_{0}^{\infty} \frac{d t}{t^{3}} e^{-M_{I}^{2} t} .
$$


According to eqs. (5.12), (5.18) and (5.20) the mass spectrum of the $b c^{\prime}$-sector takes the form

$$
M_{I}^{2}(\xi)=2 g f n+m g f+\left|M_{\eta \eta^{\prime}}\right|^{2},
$$

where $n$ is the Landau level, and $m$ takes the values $m=-1,0,1,2,3$; multiplicities $l_{m}$ and fermion numbers $F_{m}$ for different values of $m$ are $\left(l_{m}\right)=(1,4,6,4,1)$ and $\left(F_{m}\right)=$ $(0,1,0,1,0)$. The sum over Landau levels is easily carried out,

$$
\sum_{n=0}^{\infty} e^{-\left(2 g f n+g f m+\left|M_{\eta \eta^{\prime}}\right|^{2}\right) t}=e^{-\left|M_{\eta \eta^{\prime}}\right|^{2} t-g f(m-1) t} \frac{1}{2 \sinh (g f t)},
$$

and the sum over all bosons and fermions yields

$$
\begin{aligned}
\sum_{m} l_{m}(-)^{F_{m}} \sum_{n=0}^{\infty} e^{-\left(2 g f n+g f m+\left|M_{\eta \eta^{\prime}}\right|^{2}\right) t}= & e^{-\left|M_{\eta \eta^{\prime}}\right|^{2} t} \frac{1}{2 \sinh (g f t)} \\
& \times\left(e^{2 g f t}-4 e^{g f t}+6-4 e^{-g f t}+e^{-2 g f t}\right) \\
= & 16 e^{-\left|M_{\eta \eta^{\prime}}\right|^{2} t} \frac{\sinh ^{4}(g f t / 2)}{2 \sinh (g f t)} .
\end{aligned}
$$

There are two flux quanta in the first torus leading to a multiplicity two for all states. Introducing radii for the second and third torus as $\left(R_{1}, R_{2}, R_{3}, R_{4}\right)=\left(L_{2}, L_{2}^{\prime}, L_{3}, L_{3}^{\prime}\right) / 2 \pi$, the final result for the potential takes the form

$$
V(\xi)=-\frac{1}{2 \pi^{2}} \int_{0}^{\infty} \frac{d t}{t^{3}} \frac{\sinh ^{4}\left(\frac{g f t}{2}\right)}{\sinh (g f t)} \sum_{m_{i}} \exp \left(-t\left(\frac{m_{i}}{R_{i}}+g \xi_{i}\right)^{2}\right) .
$$

The integral $V(\xi)=\int d t V(t, \xi)$ has an infrared as well as an ultraviolet divergence. For large $t$ the contribution of the $m_{i}=0$ term to the integrand behaves as

$$
V(t, \xi) \propto \frac{1}{t^{3}} e^{\left(g f-g^{2} \xi^{2}\right) t} .
$$

Hence, the integral diverges if $\xi^{2}<f / g$. The same is true if $\xi$ is closer than $\sqrt{f / g}$ to any lattice vector $m / R$. This infrared divergence is an effect of the tachyon in the spectrum. Moreover, there is an ultraviolet divergence. Although each term in the sum is convergent, the sum over KK modes behaves as $R^{4} / t^{2}$ for small $t$ so that the integrand scales as

$$
V(t, \xi) \propto \frac{(g f)^{3} R^{4}}{t^{2}} e^{\left(g f-g^{2} \xi^{2}\right) t} .
$$

Introducing an ultraviolet cutoff, $t>\delta \equiv 1 / \Lambda_{\mathrm{UV}}^{2}$, the quadratic ultraviolet divergence becomes manifest, $V \sim(g f)^{3} R^{4} \Lambda_{\mathrm{UV}}^{2}$.

A convenient regularization of the potential can be obtained by considering a Poisson resummation of the sum over KK modes,

$$
\sum_{m_{i}} \exp \left(-t\left(\frac{m_{i}}{R_{i}}+g \xi_{i}\right)^{2}\right)=\frac{\pi^{2} \prod_{i} R_{i}}{t^{2}} \sum_{l_{i}} \exp \left(i \sum_{i} l_{i} R_{i} \xi_{i}-\pi^{2} \sum_{i}\left(R_{i} l_{i}\right)^{2} / t\right) .
$$


The ultraviolet divergence is now encoded in the term $l_{i}=0$. Adding to the sum a counter term

$$
-c_{1} e^{-\mu_{1}^{2} t}-c_{2} e^{-\mu_{2}^{2} t}
$$

with

$$
1-c_{1}-c_{2}=0, \quad c_{1} \mu_{1}^{2}+c_{2} \mu_{2}^{2}=0
$$

implies that

$$
\sum_{m_{i}} \exp \left(-t\left(\frac{m_{i}}{R_{i}}+g \xi_{i}\right)^{2}\right)-\frac{\pi^{2} \prod_{i} R_{i}}{t^{2}}\left(c_{1} e^{-\mu_{1}^{2} t}+c_{2} e^{-\mu_{2}^{2} t}\right)
$$

is finite as $t \rightarrow 0$, yielding a finite integral $V(\xi)=\int d t V(t, \xi)$. Note, that the terms

$$
\int \frac{d t}{t^{5}} e^{-\mu_{i}^{2} t} \propto \int \frac{d t}{t} \int \frac{d^{8} k}{(2 \pi)^{8}} e^{-\left(k^{2}+\mu_{i}^{2}\right) t} \propto \int \frac{d^{8} k}{(2 \pi)^{8}} \ln \left(k^{2}+\mu_{i}^{2}\right)
$$

correspond to Pauli-Villars regulators in 8d field theories.

Stationary points of the potential have to satisfy

$$
\frac{\partial V(t, \xi)}{\partial \xi_{i}} \propto \sum_{m_{j}}\left(\frac{m_{i}}{R_{i}}+g \xi_{i}\right) \exp \left(-t\left(\frac{m_{j}}{R_{j}}+g \xi_{j}\right)^{2}\right)=0 .
$$

The solutions are given by

$$
\hat{\xi}_{i}=\frac{n_{i}}{2 g R_{i}}, \quad n_{i} \in \mathbb{Z},
$$

since

$$
\begin{aligned}
\sum_{m_{j}}\left(\frac{m_{i}}{R_{i}}+\frac{n_{i}}{2 R_{i}}\right) & \exp \left(-t\left(\frac{m_{j}}{R_{j}}+\frac{n_{j}}{2 R_{j}}\right)^{2}\right) \\
& =\sum_{m_{j}}\left(\frac{m_{i}}{R_{i}}-\frac{n_{i}}{2 R_{i}}\right) \exp \left(-t\left(\frac{m_{j}}{R_{j}}-\frac{n_{j}}{2 R_{j}}\right)^{2}\right) \\
& =-\sum_{m_{j}}\left(\frac{m_{i}}{R_{i}}+\frac{n_{i}}{2 R_{i}}\right) \exp \left(-t\left(\frac{m_{j}}{R_{j}}+\frac{n_{j}}{2 R_{j}}\right)^{2}\right)=0 .
\end{aligned}
$$

In the vincinity of an extremum the potential can be approximated by the contributions of a few neighboring lattice points. As an example, consider a one-dimensional case where $\xi$ points in one lattice direction. For $g R \xi=1 / 2$ the four nearest points yield

$$
\begin{aligned}
& \sum_{m} \exp \left(-t\left(\frac{m}{R}+\frac{1}{2 R}\right)^{2}\right) \simeq \exp \left(-t\left(-\frac{2}{R}+\frac{1}{2 R}\right)^{2}\right) \\
& \quad+\exp \left(-t\left(-\frac{1}{R}+\frac{1}{2 R}\right)^{2}\right)+\exp \left(-t\left(\frac{1}{2 R}\right)^{2}\right)+\exp \left(-t\left(\frac{1}{R}+\frac{1}{2 R}\right)^{2}\right)+\ldots
\end{aligned}
$$

Using this approximation for the sum over KK modes the potential can be evaluated numerically. As discussed above it is periodic with period $g R \xi \sim g R \xi+1$. Tachyonic 
regions $(g R \xi-n)^{2}<g f R^{2} \equiv \Delta^{2} / 4$, where the potential is ill defined, have to be excluded. The result for the normalized potential $\hat{V}(\xi)=V(\xi) / V(\sqrt{f / g})$ is shown in figure 3 . The approximation used in eq. (6.17) is remarkably robust. Changing the number of neighboring points from four to six, or even two, does not lead to a visible change in figure 3 . At the boundary to the tachyonic region the potential looses its meaning and one has to address the problem of tachyon condensation.

The computation in the $a b$-sector goes as follows. The two stacks $a$ and $b$ intersect in the three tori, therefore in the internal magnetic field framework charged states have Landau levels in the three tori. Having checked that the mass formula (3.19) is valid in the effective field theory (though the eigenvectors are linear combination of the states in the Fock space spanned by Landau levels), one can compute the scalar potential after diagonalizing the mass matrix. The states contributing are charged gauge vectors $A_{\mu}$, three complex scalar fields $\Phi_{i}$ and four Weyl fermions $\lambda$, $\Psi_{i}$, where $i=1,2,3$. As shown in detail in section 4.2 , not all scalars in $\Phi_{i}$ are physical, some of them being absorbed by the massive Landau levels of gauge fields $A_{\mu}$. It is however simpler to consider separately the two degrees of freedom in $A_{\mu}$ and the absorbed scalars in the computation. Then the various contributions to the scalar potential are

$$
\begin{aligned}
A_{\mu}: & 2 \sum_{n_{i}} e^{-t \sum_{i}\left(2 n_{i}+1\right) g f_{i}}=\frac{1}{4 \prod_{i} \sinh \left(g f_{i} t\right)}, \\
\Phi_{i}: & 1 \sum_{n_{j} \neq n_{i}} e^{-t \sum_{j \neq i}\left(2 n_{j}+1\right) g f_{j}} \sum_{n_{i}}\left(e^{-t\left(2 n_{i}-1\right) g f_{i}}+e^{-t\left(2 n_{i}+3\right) g f_{i}}\right)=\frac{\cosh \left(2 g f_{i} t\right)}{4 \prod_{i} \sinh \left(g f_{i} t\right)}, \\
\lambda: & 1 \sum_{n_{i}}\left(e^{-2 t \sum_{i} n_{i} g f_{i}}+e^{-2 t \sum_{i}\left(n_{i}+1\right) g f_{i}}\right)=\frac{\cosh \left(g\left(f_{1}+f_{2}+f_{3}\right) t\right)}{4 \prod_{i} \sinh \left(g f_{i} t\right)}, \\
\Psi_{1}: & 1 \sum_{n_{i}}\left(e^{-2 t\left(\sum_{i} n_{i} g f_{i}+g f_{1}\right)}+e^{-2 t\left(\sum_{i}\left(n_{i}+1\right) g f_{i}-g f_{1}\right)}\right)=\frac{\cosh \left(g\left(-f_{1}+f_{2}+f_{3}\right) t\right)}{4 \prod_{i} \sinh \left(g f_{i} t\right)}
\end{aligned}
$$

with contributions of $\Psi_{2}, \Psi_{3}$ similar to the one of $\Psi_{1}$ with appropriate obvious modifications. Adding all the contributions, taking into account of the opposite sign contributions of bosons versus fermions and multiplying also by the multiplicity $I_{a b}$ of zero modes and Landau levels, one gets

$$
\begin{aligned}
V_{a b}=-\frac{I_{a b}}{16 \pi^{2}} & \int_{0}^{\infty} \frac{d t}{t^{3}} \frac{1}{\prod_{i} \sinh \left(g f_{i} t\right)}\left(1+\sum_{i} \cosh \left(2 g f_{i} t\right)\right. \\
& -\cosh \left(g\left(f_{1}+f_{2}+f_{3}\right) t\right)-\cosh \left(g\left(-f_{1}+f_{2}+f_{3}\right) t\right) \\
& \left.-\cosh \left(g\left(f_{1}-f_{2}+f_{3}\right) t\right)-\cosh \left(g\left(f_{1}+f_{2}-f_{3}\right) t\right)\right) .
\end{aligned}
$$

By using standard identities one can rewrite the result into the form

$$
\begin{aligned}
V_{a b}=\frac{I_{a b}}{2 \pi^{2}} & \int_{0}^{\infty} \frac{d t}{t^{3}} \frac{1}{\prod_{i} \sinh \left(g f_{i} t\right)} \sinh \left(\frac{g\left(f_{1}+f_{2}+f_{3}\right) t}{2}\right) \\
& \times \sinh \left(\frac{g\left(-f_{1}+f_{2}+f_{3}\right) t}{2}\right) \sinh \left(\frac{g\left(f_{1}-f_{2}+f_{3}\right) t}{2}\right) \sinh \left(\frac{g\left(f_{1}+f_{2}-f_{3}\right) t}{2}\right) .
\end{aligned}
$$




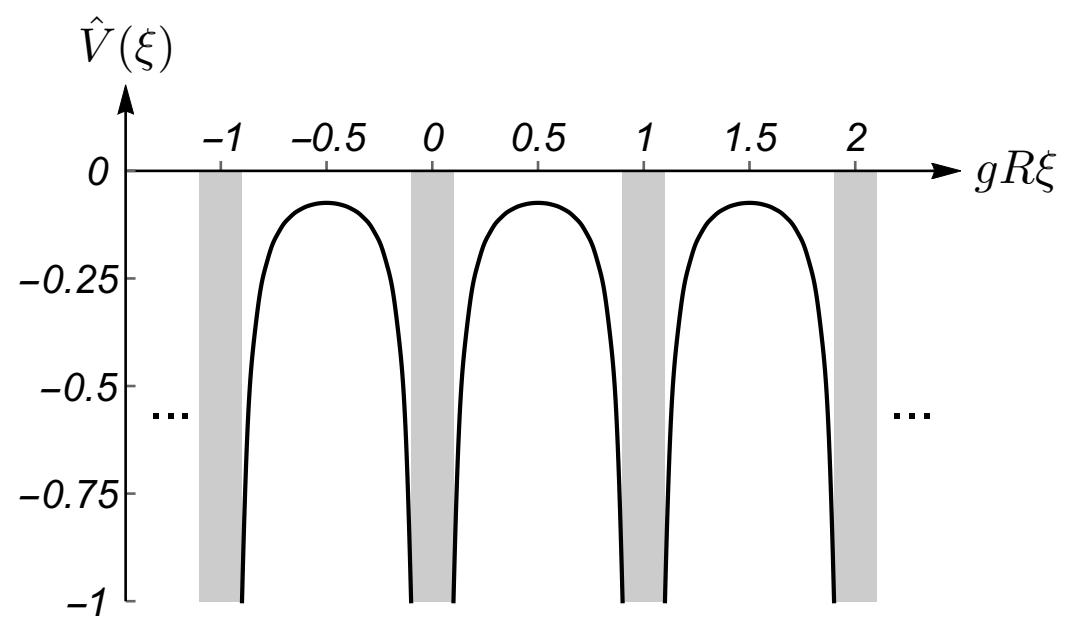

Figure 3. Wilson-line potential, normalized to its value at the border to the tachyonic region, which is chosen to have the width $\Delta=2 \sqrt{g f R^{2}}=0.2$ (see text).

Notice that the scalar potential vanishes if

$$
f_{1} \pm f_{2} \pm f_{3}=0
$$

Whenever one of the four equations (6.21) is fulfilled, supersymmetry is restored in the corresponding ( $a b$ in our case) sector, in agreement with the arguments given at the end of section 3. More precisely, if $f_{1} \pm f_{2}=0, f_{3}=0$, the four-dimensional effective theory has $\mathcal{N}=2$ supersymmetry, whereas when all $f_{i}$ are non-vanishing but one of the equations (6.21) is satisfied, the effective theory has $\mathcal{N}=1$ supersymmetry. This is not always manifest in the effective actions written in the previous sections, except for the cases when $f_{1}+f_{2}+f_{3}=0$. The reason is that for the other cases of supersymmetry restoration, the preserved supercharge generates multiplets misaligned to our superfield expansion. Indeed, in the superfield expansion we used pre-assigned superpartners in an universal way, whereas the supersymmetries preserved by the internal magnetic fields generically generate supermultiplets in a different way. While this could seem surprising at first sight, it is standard in extended supersymmetric theories (see, for example, [37-39]). One test of residual supersymmetries in the compactified theory is the boson-fermion degeneracy at each mass level. However, this is realized non-trivially, since the eigenvectors of the mass matrix mix different Landau levels, as shown explicitly in previous sections. Notice that this discussion matches known results on supersymmetry preservation in D-brane models at angles [13] and the T-dual version of type I/type II strings with internal magnetic fields. However, as far as we know, this subtlety of the effective action has never been discussed in detail in the string literature.

\subsection{String calculation}

From the string theory perspective, the scalar potential coming from various sectors is given by (minus) the cylinder partition function found by usual string quantization with 
appropriate boundary conditions, in the internal magnetic picture, or equivalently, the T-dual intersecting brane one. In particular,

$$
V_{b c^{\prime}}=-A_{b c^{\prime}}
$$

Let us start with the scalar potential in the $b c^{\prime}$-sector. The corresponding brane stacks are parallel in the second and the third torus and intersect in the first torus. Standard formulae [1] lead to the partition function

$$
A_{b c^{\prime}}=\frac{I_{b c^{\prime}}^{1}}{2\left(4 \pi^{2} \alpha^{\prime}\right)^{2}} \int_{0}^{\infty} \frac{d \tau_{2}}{\tau_{2}^{3}} \frac{\left(V_{8}-S_{8}\right)\left(\epsilon_{1} \tau \mid \tau\right)}{\eta^{6}} \frac{2 i \eta}{\theta_{1}\left(\epsilon_{1} \tau \mid \tau\right)} P_{\mathbf{m}_{\mathbf{2}}+\xi_{\mathbf{2}}} P_{\mathbf{m}_{\mathbf{3}}+\xi_{\mathbf{3}}}
$$

where

$$
P_{\mathbf{m}_{\mathbf{2}}+\xi_{\mathbf{2}}}=\sum_{\mathbf{m}_{\mathbf{2}}} e^{-\pi \tau_{2} \alpha^{\prime} \sum_{i}\left(\frac{m_{i}}{R_{i}}+\xi_{i}\right)^{2}}
$$

is the Kaluza-Klein sum along the second torus, with a similar expression for $P_{\mathbf{m}_{\mathbf{3}}+\xi_{3}}$. The parameter $\epsilon_{1}$ is related to the angle between the stacks in the first torus according to $\theta_{b c^{\prime}}^{1}=\pi \epsilon_{1}$. Various modular functions are defined in appendix D. In eq. (6.23) we also used the character

$$
\left(V_{8}-S_{8}\right)\left(\epsilon_{1} \tau \mid \tau\right) \equiv \frac{\theta_{3}^{3} \theta_{3}\left(\epsilon_{1} \tau \mid \tau\right)-\theta_{4}^{3} \theta_{4}\left(\epsilon_{1} \tau \mid \tau\right)-\theta_{2}^{3} \theta_{2}\left(\epsilon_{1} \tau \mid \tau\right)}{2 \eta^{4}}=\frac{\theta_{1}^{4}\left(\frac{\epsilon_{1} \tau}{2} \mid \tau\right)}{\eta^{4}},
$$

where the last equality is the Jacobi identity (D.5), and $\theta_{i}=\theta_{i}(0 \mid \tau)$. The modular parameter of the doubly covering torus of the cylinder is defined as

$$
q=e^{2 \pi i \tau}, \tau=\frac{i \tau_{2}}{2}
$$

and the relation with the Schwinger proper time of the field theory computation is $t=\pi \tau_{2} \alpha^{\prime}$.

The connection with the field theory computation is done by decoupling the charged open string oscillators in the formulae above, while keeping the Kaluza-Klein states and the Landau levels. This is achieved in the $\tau_{2} \rightarrow \infty$ limit of the modular functions, for example,

$$
\theta_{1}\left(\epsilon_{1} \tau \mid \tau\right) \rightarrow 2 i \sinh \left(\frac{\pi \epsilon_{1} \tau_{2}}{2}\right) q^{1 / 8},
$$

which is valid for $\left|\epsilon_{1}\right|<1 / 2$. Notice that the Wilson-line dependence of the field theory expression is accurate in the large volume limit, $v_{i} \gg \alpha^{\prime}$. Indeed, in this limit, KaluzaKlein states and Landau levels are much lighter than the charged open string oscillators. It is important that the UV divergence of the amplitude/scalar potential, which arises even after summing over all sectors due to the NSNS tadpole generated by the magnetic fields, is independent of the Wilson lines. The scalar potential can therefore be regulated by the Pauli-Villars method discussed in the previous paragraph.

The analogous expression for the amplitude $A_{b c}$ is easily found to be

$$
A_{b c}=\frac{I_{b c}^{2} I_{b c}^{3}}{2\left(4 \pi^{2} \alpha^{\prime}\right)^{2}} \int_{0}^{\infty} \frac{d \tau_{2}}{\tau_{2}^{3}} \frac{\left(V_{8}-S_{8}\right)\left(\epsilon_{2} \tau ; \epsilon_{3} \tau \mid \tau\right)}{\eta^{4}} \frac{2 i \eta}{\theta_{1}\left(\epsilon_{2} \tau \mid \tau\right)} \frac{2 i \eta}{\theta_{1}\left(\epsilon_{3} \tau \mid \tau\right)} P_{\mathbf{m}_{1}+\xi_{1}}
$$


where one can now use the Jacobi identity (D.5),

$$
\begin{aligned}
\left(V_{8}-S_{8}\right)\left(\epsilon_{2} \tau ; \epsilon_{3} \tau \mid \tau\right) \equiv & \frac{1}{2 \eta^{4}}\left(\theta_{3}^{2} \theta_{3}\left(\epsilon_{2} \tau \mid \tau\right) \theta_{3}\left(\epsilon_{3} \tau \mid \tau\right)\right. \\
& \left.\quad-\theta_{4}^{2} \theta_{4}\left(\epsilon_{2} \tau \mid \tau\right) \theta_{4}\left(\epsilon_{2} \tau \mid \tau\right)-\theta_{2}^{2} \theta_{2}\left(\epsilon_{2} \tau \mid \tau\right) \theta_{2}\left(\epsilon_{3} \tau \mid \tau\right)\right) \\
= & \frac{1}{\eta^{4}} \theta_{1}^{2}\left(\frac{\left(\epsilon_{2}+\epsilon_{3}\right) \tau}{2} \mid \tau\right) \theta_{1}^{2}\left(\frac{\left(\epsilon_{2}-\epsilon_{3}\right) \tau}{2} \mid \tau\right) .
\end{aligned}
$$

The stacks in the $a b$-sector intersect in all three tori. In this case, there are no standard Kaluza-Klein sums, but Landau levels in the three tori. The cylinder partition function reads

$$
A_{a b}=\frac{I_{a b}^{1} I_{a b}^{2} I_{a b}^{3}}{2\left(4 \pi^{2} \alpha^{\prime}\right)^{2}} \int_{0}^{\infty} \frac{d \tau_{2}}{\tau_{2}^{3}} \frac{\left(V_{8}-S_{8}\right)\left(\epsilon_{1} \tau ; \epsilon_{2} \tau ; \epsilon_{3} \tau \mid \tau\right)}{\eta^{2}} \prod_{i=1}^{3} \frac{2 i \eta}{\theta_{1}\left(\epsilon_{i} \tau \mid \tau\right)},
$$

which can again be simplified with the help of the Jacobi identity (D.5),

$$
\begin{aligned}
\left(V_{8}-S_{8}\right)\left(\epsilon_{1} \tau ; \epsilon_{2} \tau ; \epsilon_{3} \tau \mid \tau\right) \equiv & \frac{\theta_{3} \prod_{i=1}^{3} \theta_{3}\left(\epsilon_{i} \tau \mid \tau\right)-\theta_{4} \prod_{i=1}^{3} \theta_{4}\left(\epsilon_{i} \tau \mid \tau\right)-\theta_{2} \prod_{i=1}^{3} \theta_{2}\left(\epsilon_{i} \tau \mid \tau\right)}{2 \eta^{4}} \\
=-\frac{1}{\eta^{4}} \theta_{1}\left(\frac{\left(\epsilon_{1}+\epsilon_{2}+\epsilon_{3}\right) \tau}{2} \mid \tau\right) \theta_{1}\left(\frac{\left(-\epsilon_{1}+\epsilon_{2}+\epsilon_{3}\right) \tau}{2} \mid \tau\right) & \times \theta_{1}\left(\frac{\left(\epsilon_{1}-\epsilon_{2}+\epsilon_{3}\right) \tau}{2} \mid \tau\right) \theta_{1}\left(\frac{\left(\epsilon_{1}+\epsilon_{2}-\epsilon_{3}\right) \tau}{2} \mid \tau\right) .
\end{aligned}
$$

Notice that the potential vanishes whenever

$$
\epsilon_{1} \pm \epsilon_{2} \pm \epsilon_{3}=0
$$

which encode the standard condition for supersymmetry restoration (see eq. (2.12)), $\theta^{1} \pm$ $\theta^{2} \pm \theta^{3}=0$, as explained in [13].

After taking the field theory limit and by introducing Pauli-Villars regulators for the UV part of the potential and using the field theory Schwinger proper time $t$, one finds the scalar potential

$$
\begin{aligned}
V_{a b}= & \frac{I_{a b}^{1} I_{a b}^{2} I_{a b}^{3}}{2 \pi^{2}} \int_{0}^{\infty} \frac{d t}{t^{3}}\left(1-c_{1} e^{-\mu_{1}^{2} t}-c_{2} e^{-\mu_{2}^{2} t}\right) \\
& \times \frac{\sinh \left(\frac{\left(\epsilon_{1}+\epsilon_{2}+\epsilon_{3}\right) t}{4}\right) \sinh \left(\frac{\left(-\epsilon_{1}+\epsilon_{2}+\epsilon_{3}\right) t}{4}\right) \sinh \left(\frac{\left(\epsilon_{1}-\epsilon_{2}+\epsilon_{3}\right) t}{4}\right) \sinh \left(\frac{\left(\epsilon_{1}+\epsilon_{2}-\epsilon_{3}\right) t}{4}\right)}{\sinh \left(\frac{\epsilon_{1} t}{2}\right) \sinh \left(\frac{\epsilon_{2} t}{2}\right) \sinh \left(\frac{\epsilon_{3} t}{2}\right)},
\end{aligned}
$$

where $c_{1}+c_{2}=1, c_{1} \mu_{1}^{2}+c_{2} \mu_{2}^{2}=0$. The non-regularized potential matches, by using the field theory limit $\epsilon_{i} \rightarrow 2 g f_{i}$, the field theory result (6.20). As is well-known, the one-loop cylinder string partition functions can be also written, after a modular transformation, as a tree-level propagation of closed strings between two stacks of branes (see figure 4). This open-closed string duality is crucial for the consistency of the string theory partition functions. However, after taking the field theory limit and decoupling the open string massive oscillators, the field theory scalar potentials do not feature this duality. As a consequence, we choose for brevity to not write the scalar potentials in this dual formulation, which would otherwise be crucial for the full fledged string theory formulation. 

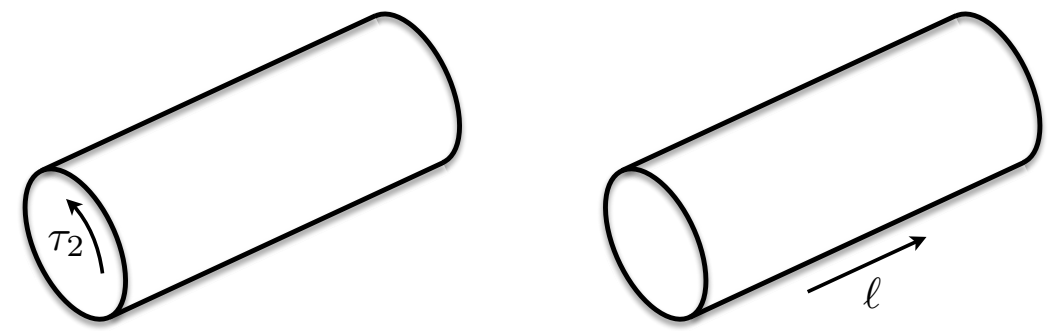

Figure 4. Left: loop-diagram for open string; right: tree-diagram for closed string.

\subsection{Volume-moduli potential}

The effective potential (6.33) depends on the parameters of $\epsilon_{i}$. In the D-brane model they represent the brane intersection angles, $\epsilon_{i}=\theta^{i} / \pi$ and in the T-dual magnetic compactification they correspond to the torus volumes $v_{i}$, with $\tan \pi \epsilon_{i}=m^{i} \rho_{i}=4 \pi^{2} \alpha^{\prime} m^{i} / v_{i}$.

Consider first the case with vanishing flux in the first torus, which is the case in the sectors $a a^{\prime}$ and $b c$. The effective potential can be obtained from eq. (6.33) by setting $\epsilon_{1}=0$, which yields

$$
V_{a a^{\prime}} \propto-\int_{0}^{\infty} \frac{d t}{t^{3}}\left(1-c_{1} e^{-\mu_{1}^{2} t}-c_{2} e^{-\mu_{2}^{2} t}\right) \frac{\sinh ^{2}\left(\frac{\left(\epsilon_{2}+\epsilon_{3}\right) t}{4}\right) \sinh ^{2}\left(\frac{\left(\epsilon_{2}-\epsilon_{3}\right) t}{4}\right)}{\sinh \left(\frac{\epsilon_{2} t}{2}\right) \sinh \left(\frac{\epsilon_{3} t}{2}\right)} .
$$

On the line $\epsilon_{2}=\epsilon_{3}$ in moduli space (see figure 2) the potential $V_{a a^{\prime}}$ vanishes. However, as one easily verifies, for $\epsilon_{2} \neq \epsilon_{3}$ the potential has an infrared divergence and approaches $-\infty$. Hence, due to the existence of a tachyon for $\epsilon_{2} \neq \epsilon_{3}$, the line $\epsilon_{2}=\epsilon_{3}$ is unstable.

We can also evaluate the integral $V_{a b}$ for non-zero fluxes in all three tori, and therefore no Wilson lines. In string theory, the result is UV divergent due to NSNS tadpoles which require a vacuum redefinition that is very challenging to perform explicitly [26].

In our field theory approach, the potential can be regulated a là Pauli-Villars, but now the result will depend on the regulator masses. We have checked numerically that for $\epsilon_{i} \ll \mu_{1,2}^{2} \ll 1 / \delta$, where $1 / \delta$ is the ultraviolet cutoff, variation of $\mu_{1,2}^{2}$ essentially changes the normalization of the potential and not the shape. Figure 5 shows the potential $V_{a b}$ for three slices of moduli space defined by $\epsilon_{3}=\hat{\epsilon}_{3}, \epsilon_{2}=\hat{\epsilon}_{2}$ and $\epsilon_{1}=\hat{\epsilon}_{1}$, where $\left(\hat{\epsilon}_{1}, \hat{\epsilon}_{2}, \hat{\epsilon}_{3}\right)=$ $(0.04,0.07,-0.1)$ is one allowed point in moduli space (see figure 5$)$. At the boundary of the tachyon-free region the potential vanishes. The figure clearly illustrates that the system is always driven to the tachyonic region in moduli space. The same conclusion has previously been reached in a related discussion in [17] from the viewpoint of the disc level scalar potential. This suggests that a stabilization mechanism for the volume moduli is needed at or above the compactification scale.

\section{Tachyon condensation}

Most sectors of the considered model have potentially tachyonic charged scalars. A frequent assumption is that such tachyonic instabilities can be avoided by means of Wilson lines. However, as we demonstrated in the previous section for the $b c^{\prime}$-sector, the one-loop 

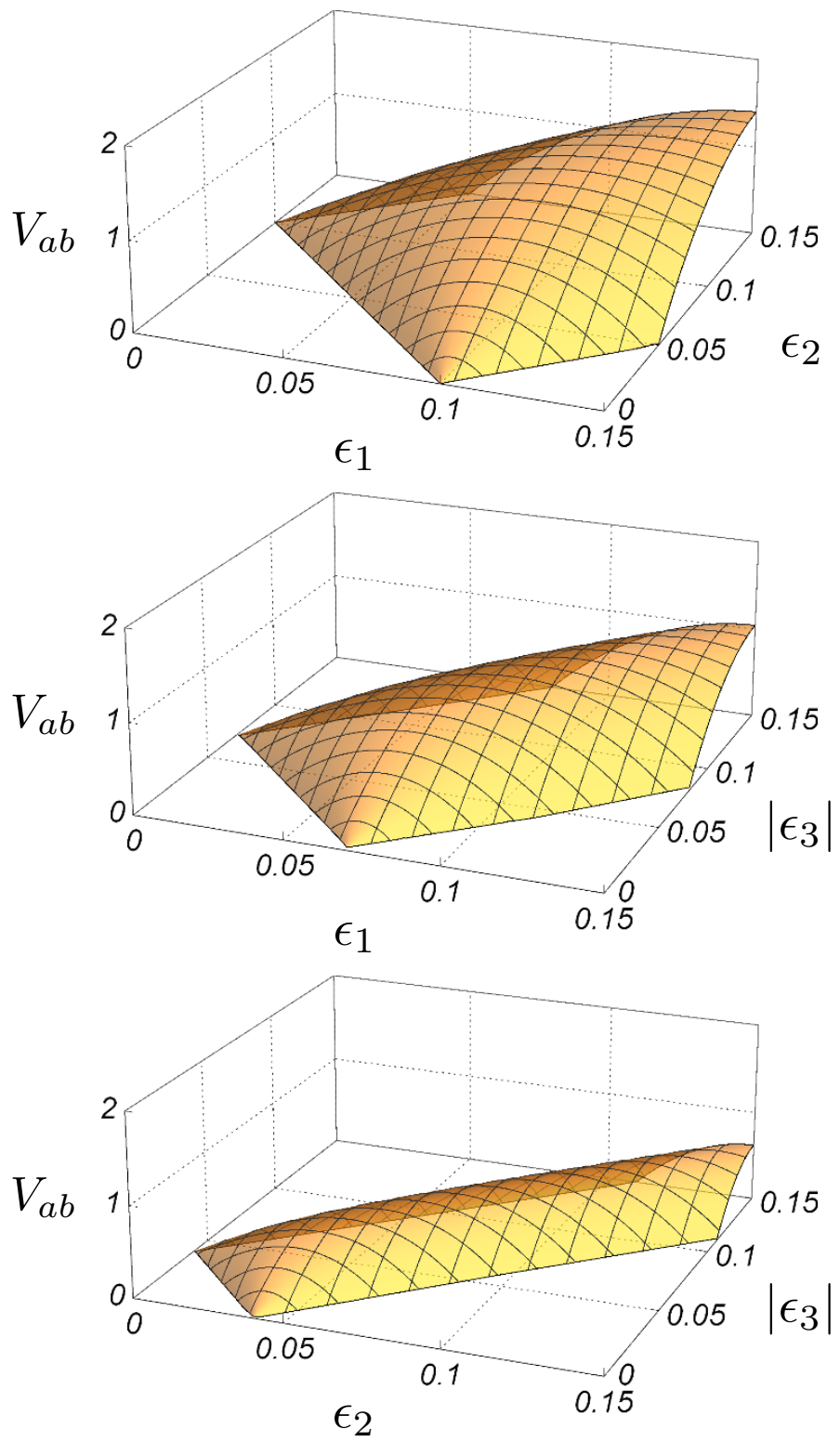

Figure 5. One-loop potential $V_{a b}$ for three slices in the three-dimensional space of the volume moduli $v_{i}$ of the three 2-tori $T_{i}^{2}$, with $\tan \pi \epsilon_{i} \propto 1 / v_{i}$. The slices are defined by $\epsilon_{3}=\hat{\epsilon}_{3}$ (top), $\epsilon_{2}=\hat{\epsilon}_{2}$ (middle), and $\epsilon_{1}=\hat{\epsilon}_{1}$ (bottom), where $\left(\hat{\epsilon}_{1}, \hat{\epsilon}_{2}, \hat{\epsilon}_{3}\right)=(0.04,0.07,-0.1)$ is a point in the tachyon-free region of moduli space. The potential (arbitrary units) is evaluated numerically for an ultraviolet cutoff $\delta^{-1}=10^{3}$ and Pauli-Villars regulator masses $\mu_{1}^{2}=75, \mu_{2}^{2}=25$.

Wilson-line potential has no stable extrema and the system is therefore driven to the tachyonic regime. For zero Wilson lines tachyon condensation takes place. This is interpreted as brane-brane recombination and it is expected that tachyon condensation restores supersymmetry, at least partially (see, for example, [34-36]). In the following, we shall address for the first time tachyon condensation in a compact space. 


\section{1 $b c^{\prime}$-sector}

The situation is particularly simple in the $b c^{\prime}$-sector. According to eq. (5.19) the field $\Phi_{0,00}^{-}=\phi_{0,00}^{1-}$ has a negative mass squared. The interesting question is whether its condensation can restore supersymmetry. Inspection of (5.13) shows that the relevant $F$ - and $D$-terms are given by (for simplicity we restrict ourselves to $\eta=\eta^{\prime}=0$ ),

$$
\begin{aligned}
-\bar{F}_{n}^{+} & =\left|M_{n, 00}\right| \Phi_{n}^{-}, \\
-2 D_{1,2} & =4 f+g \sum_{n}\left(\left|\phi_{n}^{+}\right|^{2}+\left|\Phi_{n}^{+}\right|^{2}+\left|\Xi_{n}^{+}\right|^{2}-\left|\phi_{n}^{-}\right|^{2}-\left|\Phi_{n}^{-}\right|^{2}-\left|\Xi_{n}^{-}\right|^{2}\right), \\
-\sqrt{2} D_{n}^{+} & =\left|M_{n}\right| \Xi_{n}^{+}-\left|M_{n+1}\right| \bar{\Xi}_{n}^{-} .
\end{aligned}
$$

The equation $D_{n}^{+}=0$ is easily satisfied by $\Xi_{n}^{+}=\Xi_{n}^{-}=0$. The crucial point is that because of $\left|M_{0,00}\right|=0$, the field $\Phi_{0}^{-}=\phi_{0}^{1-}$ decouples from the superpotential, and therefore $\bar{F}_{0}^{+}=0$. Setting $\phi_{n}^{+}=\Phi_{n}^{+}=\Xi_{n}^{+}=\phi_{n}^{-}=\Phi_{n+1}^{-}=\Xi_{n}^{-}=0, D_{1,2}=0$ can be satisfied by $\phi_{0}^{1-}=\sqrt{2 f / g}$, and supersymmetry is restored. The D-term scalar potential

$$
V_{D}=\frac{g}{4}\left(4 f-\left|\phi_{0}^{1-}\right|^{2}\right)^{2}
$$

yielding the tachyonic mass squared $-2 g f$, in agreement with eq. (5.19).

According to eqs. (2.7), (3.6), (3.12) and (3.15), a vev of $\phi_{0}^{1-}$ leads to masses for all chiral fermions,

$$
\mathcal{L}_{\text {mass }} \propto y\left|\phi_{0}^{1-}\right|\left(\sum_{j=1}^{3(l-2)} \overline{\boldsymbol{N}}_{1,0}^{j} \boldsymbol{N}_{0,1}^{j}+\sum_{j=1}^{l+2} \overline{\mathbf{N}}_{0,1}^{j} \boldsymbol{N}_{1,0}^{j}\right)
$$

where $j$ labels the ground state wave functions. Hence, after tachyon condensation, all fermions have masses of order $\sqrt{g f}$.

\section{$7.2 \quad b c$-sector}

This sector is very similar to the $a a^{\prime}$-sector, since the flux vanishes in the first torus. However, an important difference is the sign of the flux densities. In the $a a^{\prime}$-sector one has positive flux densities in the second and the third torus. On the contrary, in the $b c$ sector the two flux densities have opposite sign. Taking this into account, the relevant $F$ and $D$-terms can be essentially read off from eq. (4.13). One finds, before forming linear combinations for mass eigenstates,

$$
\begin{aligned}
\bar{F}_{n, n^{\prime}}^{1+-} & =\sqrt{2 g f_{2} n} \phi_{n-1, n^{\prime}}^{3-+}-\sqrt{2 g f_{3}\left(n^{\prime}+1\right)} \phi_{n, n^{\prime}+1}^{2-+}, \\
\bar{F}_{n, n^{\prime}}^{1-+}= & -\sqrt{2 g f_{2}(n+1)} \phi_{n+1, n^{\prime}}^{3+-}+\sqrt{2 g f_{3} n^{\prime}} \phi_{n, n^{\prime}-1}^{2+-}, \\
-\left(D_{1}-D_{2}\right)= & f_{2}-f_{3}+g \sum_{n, n^{\prime}}\left(\left|\phi_{n, n^{\prime}}^{i+-}\right|^{2}-\left|\phi_{n, n^{\prime}}^{i-+}\right|^{2}\right) \\
D_{n, n^{\prime}}^{-+}= & \sqrt{g f_{2}}\left(\sqrt{n} \bar{\phi}_{n-1, n^{\prime}}^{2+-}-\sqrt{n+1} \phi_{n+1, n^{\prime}}^{2-+}\right) \\
& -\sqrt{g f_{3}}\left(\sqrt{n^{\prime}+1} \bar{\phi}_{n, n^{\prime}+1}^{3+-}-\sqrt{n^{\prime}} \phi_{n, n^{\prime}-1}^{3-+}\right) .
\end{aligned}
$$


Similar to the $b c^{\prime}$-sector, now the fields $\phi_{0,0}^{2-+}$ and $\phi_{0,0}^{3+-}$ decouple from the superpotential. Setting all other fields to zero, $F_{n, n^{\prime}}^{1+-}, F_{n, n^{\prime}}^{1-+}$ and $D_{n, n^{\prime}}^{-+}$vanish and one is left with

$$
-\left(D_{1}-D_{2}\right)=f_{2}-f_{3}+g\left(\left|\phi_{0,0}^{3+-}\right|^{2}-\left|\phi_{0,0}^{2-+}\right|^{2}\right) \text {. }
$$

Depending on the sign of $f_{2}-f_{3}, D_{1}-D_{2}=0$ is achieved for a vev of $\phi_{0,0}^{3+-}$ or $\phi_{0,0}^{2-+}$. Hence, as in the $b c^{\prime}$-sector, tachyon condensation restores supersymmetry. However, according to eq. (2.7), these vev's do not generate mass terms for chiral fermions. In the special case $f_{2}=f_{3}$, there are two massless scalars and no tachyon condensation takes place.

Tachyon condensation in the $a a^{\prime}$-sector is more complicated since the $\mathrm{SU}(N)$ D-terms and the superpotential couple the antisymmetric tensor to chiral fields in the adjoint representation of $\mathrm{SU}(N)$. Also Wilson lines of the $\mathrm{U}(1)_{a}$ gauge group have to be taken into account. This allows for more complicated solutions of the $F$ - and $D$-term equations. Tachyon condensation involves fields of order $\sqrt{f / g}$. Hence, the couplings between the various sectors by $D$ - and $F$-terms have to be taken into account in a complete analysis of the vacuum structure.

\section{Conclusions and open questions}

We have studied the effective field theory for an intersecting D-brane model and its T-dual magnetic compactification, which has all features wanted for extensions of the Standard Model with high-scale supersymmetry breaking: the model has a 'matter sector' with chiral fermions, broken supersymmetry and massive scalars, and a 'Higgs sector' with vector-like fermions. For certain choices of fluxes, in some sectors scalars are massless and supersymmetry is partially preserved. Expectation values of Higgs scalars can give mass to the chiral fermions. In general it is assumed that tachyons in the Higgs sector can be avoided by means of Wilson lines. All these features are well known from phenomenological applications in the literature (see, for example, [16, 21]).

The considered model is also representative at the technical level. The different sectors are examples of the three possibilities for background gauge fields, with flux in one torus and Wilson lines in the other two, flux in two tori and Wilson lines in one torus, and flux in three tori. The magnetic flux mixes the towers of Landau levels, yielding also massless Goldstone bosons that give mass to vector fields via the Stückelberg mechanism. Physical $4 \mathrm{~d}$ fields are linear combinations of fields from different Landau levels. For each mass level the counting of bosonic and fermionic states is consistent with the string mass formula.

The scalar masses depend on moduli, i.e., Wilson lines and the volume moduli of the three tori. One of the main results of this paper is the computation of the one-loop effective potential for Wilson lines in the 'Higgs sector' based on the effective $4 \mathrm{~d}$ field theory. Summing over the tower of Landau levels leads to a result which is consistent with the string cylinder amplitude in the field theory limit. It turns out that the computation of the string amplitude is very convenient to obtain the one-loop potential, and in this way we have therefore evaluated the contributions of all sectors of the model to the effective potential. 
Notice, that in string theory, whenever the magnetic fluxes break supersymmetry, there are NSNS tadpoles that generate divergences. These divergences, that are UV from the loop viewpoint, are actually IR from the viewpoint of the tree-level gravitational exchange. Their existence implies that the computation is not performed in the right vacuum, that has to be redefined (see, for example, [26]), which is technically very challenging (for recent progress, see, for example, [40]). This does not affect the Wilson-line potential, since the divergence is independent of the Wilson lines. Our field theory approach with Pauli-Villars regulators allowed us to analyze also the dependence of the potential on the volume moduli. We find the expected instability of the perturbative vacuum. However, a more detailed study is needed to obtain a definite result on the potential vacuum instability.

The one-loop Wilson-line potential in the Higgs sector is concave. There are no stable extrema and the system is therefore driven to the tachyonic regime. We showed that for vanishing Wilson lines tachyon condensation indeed takes place, and the corresponding vacuum expectation value gives masses to all chiral fermions of the order of the compactification scale. It is quite possible, however, that in other models some chirality remains after tachyon condensation.

As we have seen, tachyon condensation in the Higgs sector restores supersymmetry. It is important to extend the first analysis in this paper to all sectors of the model, since the restoration of supersymmetry is closely related to the vacuum energy density and the stability, or possibly metastability, of the model. Given the phenomenological virtues of magnetic compactifications and intersecting D-brane models, it appears mandatory to further pursue these questions.

\section{Acknowledgments}

We thank Ralph Blumenhagen, Luis Ibáñez, C.S. Lim, Dieter Lüst, Hans-Peter Nilles, Augusto Sagnotti and especially Markus Dierigl for valuable discussions. E.D. was supported in part by the "Agence Nationale de la Recherche" (ANR). Y.T. is supported in part by Grants-in-Aid for JSPS Overseas Research Fellow (No. 18J60383) from the Ministry of Education, Culture, Sports, Science and Technology in Japan.

\section{A Embedding $\mathrm{U}(N)$ into $\mathrm{SO}(2 N)$}

In section 2 and section 3 we discussed an intersection D-brane model with gauge group $\mathrm{U}(14) \times \mathrm{U}(1) \times \mathrm{U}(1)$ and a T-dual type I string compactification on a magnetized torus, respectively. The connection becomes particularly transparent if one uses step generators for the $\mathrm{U}(16)$ subgroup of $\mathrm{SO}(32)$. In this appendix we collect some formulae which extend the step generators of a $\mathrm{U}(N)$ algebra to an $\mathrm{SO}(2 N)$ algebra by adding generators that transform as the antisymmetric complex representation of $\mathrm{U}(N)$.

The $N^{2}$ generators of $\mathrm{U}(N)$ are given by matrices $\hat{T}_{\alpha \beta}$ that transform as $N \otimes \bar{N}$,

$$
\left(\hat{T}_{\alpha \beta}\right)_{\alpha^{\prime} \bar{\beta}^{\prime}}=\delta_{\alpha^{\prime} \alpha} \delta_{\beta \bar{\beta}^{\prime}}
$$


Note that the $\hat{T}_{\alpha \beta}$ are not hermitian but satisfy the relation

$$
\hat{T}_{\alpha \beta}^{T}=\hat{T}_{\beta \alpha} .
$$

The step generators are related to $N(N+1) / 2$ symmetric hermitean generators $\hat{T}_{\alpha \beta}^{1}$ and $N(N-1) / 2$ antisymmetric hermitian generators $\hat{T}_{\alpha \beta}^{2}$ by

$$
\hat{T}_{\alpha \beta}^{1}=\hat{T}_{\alpha \beta}+\hat{T}_{\beta \alpha}, \quad \hat{T}_{\alpha \beta}^{2}=i\left(\hat{T}_{\alpha \beta}-\hat{T}_{\beta \alpha}\right)
$$

Infinitesimal $\mathrm{U}(N)$ transformations of the fundamental representation $\psi \sim N$ read

$$
\delta \psi=i\left(\varepsilon_{\alpha \beta} \hat{T}_{\alpha \beta}+\varepsilon_{\alpha \beta}^{*} \hat{T}_{\beta \alpha}\right) \psi=i\left(\varepsilon_{\alpha \beta}^{1} \hat{T}_{\alpha \beta}^{1}+\varepsilon_{\alpha \beta}^{2} \hat{T}_{\alpha \beta}^{2}\right) \psi,
$$

where $\varepsilon_{\alpha \beta}=\varepsilon_{\alpha \beta}^{1}+i \varepsilon_{\alpha \beta}^{2}$. Note that $\varepsilon_{\alpha \beta}^{1} \hat{T}_{\alpha \beta}^{1}$ and $\varepsilon_{\alpha \beta}^{2} \hat{T}_{\alpha \beta}^{2}$ are symmetric and antisymmetric $N \times N$ matrices, respectively. An infinitesimal transformation of the complex conjugate representation $\bar{\psi} \sim \bar{N}$ reads

$$
\delta \bar{\psi}=-i\left(\varepsilon_{\alpha \beta}^{*} \hat{T}_{\alpha \beta}+\varepsilon_{\alpha \beta} \hat{T}_{\beta \alpha}\right) \bar{\psi}=-i\left(\varepsilon_{\alpha \beta}^{1} \hat{T}_{\alpha \beta}^{1}+\varepsilon_{\alpha \beta}^{2} \hat{T}_{\alpha \beta}^{2}\right) \bar{\psi} .
$$

The step generators satisfy the commutator relations

$$
\left[\hat{T}_{\alpha \beta}, \hat{T}_{\gamma \delta}\right]=\delta_{\beta \gamma} \hat{T}_{\alpha \delta}-\delta_{\delta \alpha} \hat{T}_{\gamma \beta}
$$

and are normalized as

$$
\operatorname{tr}\left(\hat{T}_{\alpha \beta}\right)=\delta_{\alpha \beta}, \quad \operatorname{tr}\left(\hat{T}_{\alpha \beta}^{T} \hat{T}_{\gamma \delta}\right)=\delta_{\alpha \gamma} \delta_{\beta \delta} .
$$

The $N \times N$ matrices $\hat{T}_{\alpha \beta}$ and $-\hat{T}_{\beta \alpha}$ can be combined into $2 N \times 2 N$ matrices

$$
T_{\alpha \beta}=\left(\begin{array}{cc}
\hat{T}_{\alpha \beta} & 0 \\
0 & -\hat{T}_{\beta \alpha}
\end{array}\right)=\left(\begin{array}{cc}
\hat{T}_{\alpha \beta} & 0 \\
0 & -\hat{T}_{\alpha \beta}^{T}
\end{array}\right),
$$

which act on the $2 N$-component vector

$$
\Psi=\left(\begin{array}{c}
\psi \\
\bar{\psi}
\end{array}\right)
$$

Note that

$$
\operatorname{tr}\left(T_{\alpha \beta}\right)=0, \quad \operatorname{tr}\left(T_{\alpha \beta}^{\dagger} T_{\gamma \delta}\right)=2 \delta_{\alpha \gamma} \delta_{\beta \delta} .
$$

The generators $T_{\alpha \beta}$ satisfy the same algebra as the generators $\hat{T}_{\alpha \beta}$,

$$
\left[T_{\alpha \beta}, T_{\gamma \delta}\right]=\delta_{\beta \gamma} T_{\alpha \delta}-\delta_{\delta \alpha} T_{\gamma \beta},
$$

and the corresponding $\mathrm{SO}(2 N)$ transformations read

$$
\delta \Psi=i\left(\varepsilon_{\alpha \beta}^{1} T_{\alpha \beta}^{1}+\varepsilon_{\alpha \beta}^{2} T_{\alpha \beta}^{2}\right) \Psi .
$$


The generators of $\mathrm{SO}(2 N) / \mathrm{U}(N)$ form a complex antisymmetric tensor of $\mathrm{U}(N)$. They can be chosen as

$$
X_{\gamma \delta}^{+}=\left(\begin{array}{cc}
0 & \hat{X}_{\gamma \delta} \\
0 & 0
\end{array}\right), \quad X_{\gamma \delta}^{-}=\left(\begin{array}{cc}
0 & 0 \\
-\hat{X}_{\gamma \delta} & 0
\end{array}\right),
$$

where

$$
\left(\hat{X}_{\gamma \delta}\right)_{\gamma^{\prime} \delta^{\prime}}=\delta_{\gamma \gamma^{\prime}} \delta_{\delta \delta^{\prime}}-\delta_{\gamma \delta^{\prime}} \delta_{\delta \gamma^{\prime}}
$$

with

$$
\hat{X}_{\gamma \delta}=-\hat{X}_{\gamma \delta}^{T}=-\hat{X}_{\gamma \delta}^{\dagger}=-\hat{X}_{\delta \gamma} .
$$

The generators $X_{\gamma \delta}^{ \pm}$satisfy the relations

$$
X_{\gamma \delta}^{+\dagger} X_{\epsilon \rho}^{-}=X_{\gamma \delta}^{-\dagger} X_{\epsilon \rho}^{+}=0,
$$

and are normalized as

$$
\operatorname{tr}\left(X_{\gamma \delta}^{ \pm}{ }^{\dagger} X_{\epsilon \rho}^{ \pm}\right)=2\left(\delta_{\gamma \epsilon} \delta_{\delta \rho}-\delta_{\gamma \rho} \delta_{\delta \epsilon}\right) .
$$

Together with $T_{\alpha \beta}$ they form a closed algebra,

$$
\begin{aligned}
& {\left[T_{\alpha \beta}, X_{\gamma \delta}^{+}\right]=\delta_{\beta \gamma} X_{\alpha \delta}^{+}+\delta_{\beta \delta} X_{\gamma \alpha}^{+},} \\
& {\left[T_{\alpha \beta}, X_{\gamma \delta}^{-}\right]=-\delta_{\alpha \gamma} X_{\beta \delta}^{-}-\delta_{\alpha \delta} X_{\gamma \beta}^{-},} \\
& {\left[X_{\gamma \delta}^{+}, X_{\epsilon \rho}^{+}\right]=\left[X_{\gamma \delta}^{-}, X_{\epsilon \rho}^{-}\right]=0,} \\
& {\left[X_{\gamma \delta}^{+}, X_{\epsilon \rho}^{-}\right]=\delta_{\gamma \epsilon} T_{\delta \rho}-\delta_{\delta \epsilon} T_{\gamma \rho}+\delta_{\delta \rho} T_{\gamma \epsilon}-\delta_{\gamma \rho} T_{\delta \epsilon} .}
\end{aligned}
$$

The corresponding $\mathrm{SO}(2 N)$ transformations read

$$
\delta \Psi=i\left(\tilde{\varepsilon}_{\gamma \delta} X_{\gamma \delta}^{+}+\tilde{\varepsilon}_{\gamma \delta}^{*} X_{\gamma \delta}^{-}\right) \Psi=i\left(\tilde{\varepsilon}_{\gamma \delta}^{1} X_{\gamma \delta}^{1}+\tilde{\varepsilon}_{\gamma \delta}^{2} X_{\gamma \delta}^{2}\right) \Psi,
$$

where $\tilde{\varepsilon}_{\gamma \delta}=\tilde{\varepsilon}_{\gamma \delta}^{1}+i \tilde{\varepsilon}_{\gamma \delta}^{2}$ and

$$
\begin{aligned}
& X_{\gamma \delta}^{1}=X_{\gamma \delta}^{+}+X_{\gamma \delta}^{-}=\left(\begin{array}{cc}
0 & \hat{X}_{\gamma \delta} \\
-\hat{X}_{\gamma \delta} & 0
\end{array}\right), \\
& X_{\gamma \delta}^{2}=i\left(X_{\gamma \delta}^{+}-X_{\gamma \delta}^{-}\right)=i\left(\begin{array}{cc}
0 & \hat{X}_{\gamma \delta} \\
\hat{X}_{\gamma \delta} & 0
\end{array}\right) .
\end{aligned}
$$

From eqs. (A.12) and (A.19) one concludes that a general $\mathrm{SO}(2 N)$ transformation is given by the $2 N \times 2 N$ matrix

$$
X=\left(\begin{array}{cc}
S+i A_{3} & A_{1}+i A_{2} \\
-A_{1}+i A_{2} & -S+i A_{3}
\end{array}\right) .
$$

Here $S=\varepsilon_{\alpha \beta}^{1} \hat{T}_{\alpha \beta}^{1}$ is a real symmetric $N \times N$ matrix, and $A_{3}=-i \varepsilon_{\alpha \beta}^{2} \hat{T}_{\alpha \beta}^{2}, A_{1}=\tilde{\varepsilon}_{\gamma \delta}^{1} \hat{X}_{\gamma \delta}$ and $A_{2}=\tilde{\varepsilon}_{\gamma \delta}^{2} \hat{X}_{\gamma \delta}$ are real antisymmetric $N \times N$ matrices. This can be compared to the standard form of $\mathrm{SO}(2 N)$ generators [41]

$$
\lambda=-i\left(\begin{array}{cc}
\eta_{1} & \rho \\
-\rho^{T} & \eta_{2}
\end{array}\right)=-\lambda^{T},
$$


where $\eta_{1}$ and $\eta_{2}$ are antisymmetric real $N \times N$ matrices and $\rho$ is an arbitrary real $N \times N$ matrix. After a unitary transformation,

$$
U=\frac{1}{\sqrt{2}}\left(\begin{array}{cc}
I & -i I \\
I & i I
\end{array}\right)
$$

one obtains

$$
\lambda^{\prime}=U \lambda U^{\dagger}=\frac{1}{2}\left(\begin{array}{c}
\rho+\rho^{T}-i\left(\eta_{1}+\eta_{2}\right)-\left(\rho-\rho^{T}\right)-i\left(\eta_{1}-\eta_{2}\right) \\
\left(\rho-\rho^{T}\right)-i\left(\eta_{1}-\eta_{2}\right)-\left(\rho+\rho^{T}\right)-i\left(\eta_{1}+\eta_{2}\right)
\end{array}\right) .
$$

This expression for $\lambda^{\prime}$ agrees with the one for $X$ in eq. (A.21) with $S=\left(\rho+\rho^{T}\right) / 2$, $A_{3}=-\left(\eta_{1}+\eta_{2}\right) / 2, A_{1}=-\left(\rho-\rho^{T}\right) / 2$ and $A_{2}=-\left(\eta_{1}-\eta_{2}\right) / 2$.

Notice that the transformation (A.23) is also diagonalizing the magnetic flux. Indeed, in the $\mathrm{SO}(2 N)$ basis, the magnetic flux is of the type

$$
\langle F\rangle=\left(\begin{array}{cc}
0 & I \\
-I & 0
\end{array}\right)
$$

After the unitary transformation, the flux becomes

$$
U\langle F\rangle U^{\dagger}=\left(\begin{array}{cc}
i I & 0 \\
0 & -i I
\end{array}\right) .
$$

\section{B Commutators}

In sections $2-5$ we have considered the groups $G=\mathrm{SO}(2(N+2)) \supset \mathrm{U}(N) \times \mathrm{U}(1) \times \mathrm{U}(1)=$ $H$, and in eqs. (3.11), (3.12) and (3.13) we have expanded vector, chiral and anti-chiral superfields in terms of $\mathrm{SO}(2(N+2))$ generators, with the identifications (cf. (3.6)),

$$
H_{0}=\frac{1}{\sqrt{N}} T_{\alpha \alpha}, \quad H_{1}=T_{N+1, N+1}, \quad H_{2}=T_{N+2, N+2}, \quad T_{\alpha \beta}=\tilde{T}_{\alpha \beta}+\frac{1}{\sqrt{N}} \delta_{\alpha \beta} H_{0}
$$

for generators of $H$ and

$$
\begin{aligned}
& T_{\alpha}^{-0}=T_{\alpha, N+1}, \quad T_{\alpha}^{0-}=T_{\alpha, N+2}, \quad T_{\alpha}^{+0}=T_{N+1, \alpha}, \quad T_{\alpha}^{0+}=T_{N+2, \alpha}, \quad T^{+-}=T_{N+1, N+2}, \\
& T^{-+}=T_{N+2, N+1}, \quad X_{\alpha}^{+0}=X_{\alpha, N+1}^{+}=-X_{N+1, \alpha}^{+}, \quad X_{\alpha}^{0+}=X_{\alpha, N+2}^{+}=-X_{N+2, \alpha}^{+}, \\
& X_{\alpha}^{-0}=X_{N+1, \alpha}^{-}=-X_{\alpha, N+1}^{-}, \quad X_{\alpha}^{0-}=X_{N+2, \alpha}^{-}=-X_{\alpha, N+2}^{-}, \\
& X^{++}=X_{N+1, N+2}^{+}=-X_{N+2, N+1}^{+}, \quad X^{--}=X_{N+2, N+1}^{-}=-X_{N+1, N+2}^{-},
\end{aligned}
$$

for generators of $G / H$.

Non-vanishing commutators needed in sections $3-5$ include

$$
\begin{aligned}
& {\left[H_{0}, T_{\alpha}^{\mp 0}\right]= \pm \frac{1}{\sqrt{N}} T_{\alpha}^{\mp 0}, \quad\left[H_{1}, T_{\alpha}^{\mp 0}\right]=\mp T_{\alpha}^{\mp 0}, \quad\left[T_{\alpha}^{-0}, T_{\beta}^{+0}\right]=T_{\alpha \beta}-\delta_{\alpha \beta} H_{1},} \\
& {\left[H_{0}, T_{\alpha}^{0 \mp}\right]= \pm \frac{1}{\sqrt{N}} T_{\alpha}^{0 \mp}, \quad\left[H_{2}, T_{\alpha}^{0 \mp}\right]=\mp T_{\alpha}^{0 \mp}, \quad\left[T_{\alpha}^{0-}, T_{\beta}^{0+}\right]=T_{\alpha \beta}-\delta_{\alpha \beta} H_{2},}
\end{aligned}
$$




$$
\begin{aligned}
{\left[H_{0}, X_{\alpha}^{ \pm 0}\right] } & = \pm \frac{1}{\sqrt{N}} X_{\alpha}^{ \pm 0}, \quad\left[H_{1}, X_{\alpha}^{ \pm 0}\right]= \pm X_{\alpha}^{ \pm 0}, \quad\left[X_{\alpha}^{+0}, X_{\beta}^{-0}\right]=-T_{\alpha \beta}-\delta_{\alpha \beta} H_{1} \\
{\left[H_{0}, X_{\alpha}^{0 \pm}\right] } & = \pm \frac{1}{\sqrt{N}} X_{\alpha}^{0 \pm}, \quad\left[H_{2}, X_{\alpha}^{0 \pm}\right]= \pm X_{\alpha}^{0 \pm}, \quad\left[X_{\alpha}^{0+}, X_{\beta}^{0-}\right]=-T_{\alpha \beta}-\delta_{\alpha \beta} H_{2} \\
{\left[H_{0}, X_{\alpha \beta}^{ \pm}\right] } & = \pm \frac{2}{\sqrt{N}} X_{\alpha \beta}^{ \pm}, \quad\left[X_{\alpha \beta}^{+}, X_{\gamma \delta}^{-}\right]=\delta_{\alpha \gamma} T_{\beta \delta}-\delta_{\beta \gamma} T_{\alpha \delta}+\delta_{\beta \delta} T_{\alpha \gamma}-\delta_{\alpha \delta} T_{\beta \gamma}, \\
{\left[H_{1}, T^{ \pm \mp}\right] } & = \pm T^{ \pm \mp}, \quad\left[H_{2}, T^{ \pm \mp}\right]=\mp T^{ \pm \mp}, \quad\left[T^{+-}, T^{-+}\right]=H_{1}-H_{2} \\
{\left[H_{1}, X^{ \pm \pm}\right] } & = \pm X^{ \pm \pm}, \quad\left[H_{2}, X^{ \pm \pm}\right]= \pm X^{ \pm \pm}, \quad\left[X^{++}, X^{--}\right]=H_{1}+H_{2} \\
{\left[T_{\alpha}^{-0}, T_{\beta}^{0+}\right] } & =-\delta_{\alpha \beta} T^{-+}, \quad\left[T_{\alpha}^{-0}, T^{+-}\right]=T_{\alpha}^{0-}, \quad\left[T_{\alpha}^{-0}, X_{\beta \gamma}^{-}\right]=-\delta_{\alpha \beta} X_{\gamma}^{-0}+\delta_{\alpha \gamma} X_{\beta}^{-0} \\
{\left[T_{\alpha}^{-0}, X_{\beta}^{+0}\right] } & =-X_{\alpha \beta}^{+}, \quad\left[T_{\alpha}^{-0}, X_{\beta}^{0-}\right]=-\delta_{\alpha \beta} X^{--}, \quad\left[T_{\alpha}^{-0}, X^{++}\right]=X_{\alpha}^{0+}, \\
{\left[T_{\alpha}^{0-}, T_{\beta}^{+0}\right] } & =-\delta_{\alpha \beta} T^{+-}, \quad\left[T_{\alpha}^{0-}, T^{-+}\right]=T_{\alpha}^{-0},\left[T_{\alpha}^{0-}, X_{\beta \gamma}^{-}\right]=-\delta_{\alpha \beta} X_{\gamma}^{0-}+\delta_{\alpha \gamma} X_{\beta}^{0-} \\
{\left[T_{\alpha}^{0-}, X_{\beta}^{0+}\right] } & =-X_{\alpha \beta}^{+}, \quad\left[T_{\alpha}^{0-}, X_{\beta}^{-0}\right]=\delta_{\alpha \beta} X^{--}, \quad\left[T_{\alpha}^{0-}, X^{++}\right]=-X_{\alpha}^{+0}, \\
{\left[T_{\alpha}^{+0}, T^{-+}\right] } & =-T_{\alpha}^{0+}, \quad\left[T_{\alpha}^{+0}, X_{\beta \gamma}^{+}\right]=\delta_{\alpha \gamma} X_{\beta}^{+0}-\delta_{\alpha \beta} X_{\gamma}^{+0}, \quad\left[T_{\alpha}^{+0}, X_{\beta}^{0+}\right]=\delta_{\alpha \beta} X^{++} \\
{\left[X_{\alpha}^{-0}, X_{\beta \gamma}^{++}\right] } & =\delta_{\alpha \beta} T_{\gamma}^{-0}-\delta_{\alpha \gamma} T_{\beta}^{-0}, \quad\left[X_{\alpha}^{-0}, X^{++}\right]=-T_{\alpha}^{0+}, \\
{\left[T_{\alpha}^{+0}, X_{\beta}^{-0}\right] } & =-X_{\alpha \beta}^{+}, \quad\left[T_{\alpha}^{+0}, X^{--}\right]=-X_{\alpha}^{0-}, \\
{\left[T_{\alpha}^{0+}, T^{+-}\right] } & =-T_{\alpha}^{+0}, \quad\left[T_{\alpha}^{0+}, X_{\beta \gamma}^{+}\right]=\delta_{\alpha \gamma} X_{\beta}^{0+}-\delta_{\alpha \gamma} T_{\beta}^{0-}, \quad\left[X_{\alpha}^{0-}, X^{++}\right]=T_{\alpha}^{+0} . \\
{\left[T_{\alpha}^{0+}, X_{\beta}^{0-}\right] } & =-X_{\alpha \beta}^{-}, \quad\left[T_{\alpha}^{0+}, X^{--}\right]=X_{\alpha}^{-0}, \\
{\left[T^{+-}, X_{\alpha}^{0+}\right] } & =X_{\alpha}^{+0}, \quad\left[T^{+-}, X_{\alpha}^{-0}\right]=-X_{\alpha}^{0-}, \\
{\left[T^{-+}, X_{\alpha}^{+0}\right] } & =X_{\alpha}^{0+}, \quad\left[T^{-+}, X_{\alpha}^{0-}\right]=-X_{\alpha}^{-0}, \\
{\left[X_{\beta \gamma}^{-}\right] } & =\delta_{\alpha \beta} T_{\gamma}^{+0}-\delta_{\alpha \gamma} T_{\beta}^{+0}, \quad\left[X_{\alpha}^{+0}, X_{\beta}^{0-}\right]=-\delta_{\alpha \beta} T^{+-}, \quad\left[X_{\alpha}^{+0}, X^{--}\right]=T_{\alpha}^{0-}
\end{aligned}
$$

\section{Superfield components}

For $\mathcal{N}=1$ superfields we use the conventions of Wess and Bagger [32]. In the following we list a couple of formulae for charged superfields ${ }^{10}$ that are frequently needed in the

\footnotetext{
${ }^{10}$ Note, that we use the notation $\bar{\lambda}^{+}=\overline{\lambda^{+}}$, etc.
} 
derivation of the $4 \mathrm{~d}$ effective Lagrangian:

$$
\begin{aligned}
V^{ \pm} & =-\theta \sigma^{\mu} \bar{\theta} A_{\mu}^{ \pm}+i \theta \theta \overline{\theta \lambda^{\mp}}-i \overline{\theta \theta} \theta \lambda^{ \pm}+\frac{1}{2} \theta \theta \overline{\theta \theta} D^{ \pm}, \\
\phi^{ \pm} & =\phi^{ \pm}+\sqrt{2} \theta \psi^{ \pm}+i \theta \sigma^{\mu} \bar{\theta} \partial_{\mu} \phi^{ \pm}+\ldots \\
V^{+} V^{-} & =-\frac{1}{2} \theta \theta \overline{\theta \theta} A_{\mu}^{+} A^{-\mu}+\ldots, \\
W^{+} W^{-} & =\theta \theta D^{+} D^{-}+\ldots, \\
V^{+} \phi^{-} & =\frac{i}{2} \theta \theta \overline{\theta \theta} A_{\mu}^{+} \partial^{\mu} \phi^{-}+\frac{i}{\sqrt{2}} \theta \theta \overline{\theta \theta} \lambda^{+} \psi^{-}+\frac{1}{2} \theta \theta \overline{\theta \theta} D^{+} \phi^{-}+\ldots, \\
V^{+} \bar{\phi}^{+} & =-\frac{i}{2} \theta \theta \overline{\theta \theta} A_{\mu}^{+} \partial^{\mu} \bar{\phi}^{+}-\frac{i}{\sqrt{2}} \theta \theta \overline{\theta \theta \lambda} \bar{\psi}^{+}+\frac{1}{2} \theta \theta \overline{\theta \theta} D^{+} \bar{\phi}^{+}+\ldots
\end{aligned}
$$

\section{Jacobi functions}

For the reader's convenience we collect in this appendix the definitions, transformation properties and some identities among the modular functions that are used in the text. The Dedekind function is defined by the usual product formula (with $q=e^{2 \pi i \tau}$ )

$$
\eta(\tau)=q^{\frac{1}{24}} \prod_{n=1}^{\infty}\left(1-q^{n}\right)
$$

whereas the Jacobi $\theta$-functions with general characteristic and arguments are

$$
\theta\left[\begin{array}{l}
\alpha \\
\beta
\end{array}\right](z \mid \tau)=\sum_{n \in \mathbb{Z}} e^{i \pi \tau(n-\alpha)^{2}} e^{2 \pi i(z-\beta)(n-\alpha)} .
$$

We give also the product formulae for the four special $\theta$-functions

$$
\begin{aligned}
& \theta_{1}(z \mid \tau) \equiv \theta\left[\begin{array}{c}
\frac{1}{2} \\
\frac{1}{2}
\end{array}\right](z \mid \tau)=2 q^{1 / 8} \sin \pi z \prod_{n=1}^{\infty}\left(1-q^{n}\right)\left(1-q^{n} e^{2 \pi i z}\right)\left(1-q^{n} e^{-2 \pi i z}\right) \\
& \theta_{2}(z \mid \tau) \equiv \theta\left[\begin{array}{c}
\frac{1}{2} \\
0
\end{array}\right](z \mid \tau)=2 q^{1 / 8} \cos \pi z \prod_{n=1}^{\infty}\left(1-q^{n}\right)\left(1+q^{n} e^{2 \pi i z}\right)\left(1+q^{n} e^{-2 \pi i z}\right) \\
& \theta_{3}(z \mid \tau) \equiv \theta\left[\begin{array}{l}
0 \\
0
\end{array}\right](z \mid \tau)=\prod_{n=1}^{\infty}\left(1-q^{n}\right)\left(1+q^{n-1 / 2} e^{2 \pi i z}\right)\left(1+q^{n-1 / 2} e^{-2 \pi i z}\right) \\
& \theta_{4}(z \mid \tau) \equiv \theta\left[\begin{array}{l}
0 \\
\frac{1}{2}
\end{array}\right](z \mid \tau)=\prod_{n=1}^{\infty}\left(1-q^{n}\right)\left(1-q^{n-1 / 2} e^{2 \pi i z}\right)\left(1-q^{n-1 / 2} e^{-2 \pi i z}\right)
\end{aligned}
$$

The modular properties of these functions are described by

$$
\begin{array}{ll}
\eta(\tau+1)=e^{i \pi / 12} \eta(\tau), & \theta\left[\begin{array}{l}
\alpha \\
\beta
\end{array}\right](z \mid \tau+1)=e^{-i \pi \alpha(\alpha-1)} \theta\left[\begin{array}{c}
\alpha \\
\alpha+\beta-\frac{1}{2}
\end{array}\right](z \mid \tau) \\
\eta(-1 / \tau)=\sqrt{-i \tau} \eta(\tau), & \theta\left[\begin{array}{l}
\alpha \\
\beta
\end{array}\right]\left(\frac{z}{\tau} \mid \frac{-1}{\tau}\right)=\sqrt{-i \tau} e^{2 i \pi \alpha \beta+i \pi z^{2} / \tau} \theta\left[\begin{array}{c}
\beta \\
-\alpha
\end{array}\right](z \mid \tau) .
\end{array}
$$


A useful identity for theta functions is the Jacobi identity

$$
\begin{aligned}
\sum_{\alpha, \beta=0,1 / 2}(-1)^{2 \alpha+2 \beta+4 \alpha \beta} \prod_{i=1}^{4} \theta\left[\begin{array}{l}
\alpha \\
\beta
\end{array}\right]\left(z_{i} \mid \tau\right)= \\
-2 \theta_{1}\left(\frac{-z_{1}+z_{2}+z_{3}+z_{4}}{2} \mid \tau\right) \theta_{1}\left(\frac{z_{1}-z_{2}+z_{3}+z_{4}}{2} \mid \tau\right) \\
\quad \times \theta_{1}\left(\frac{z_{1}+z_{2}-z_{3}+z_{4}}{2} \mid \tau\right) \theta_{1}\left(\frac{z_{1}+z_{2}+z_{3}-z_{4}}{2} \mid \tau\right) .
\end{aligned}
$$

In computing partition functions, it is useful to define $\mathrm{SO}(2 n)$ characters. Of particular relevance for us are

$$
\begin{aligned}
& V_{8}\left(z_{i} \tau \mid \tau\right)=\frac{\prod_{i=1}^{4} \theta_{3}\left(z_{i} \tau \mid \tau\right)-\prod_{i=1}^{4} \theta_{4}\left(z_{i} \tau \mid \tau\right)}{2 \eta^{4}}, \\
& S_{8}\left(z_{i} \tau \mid \tau\right)=\frac{\prod_{i=1}^{4} \theta_{2}\left(z_{i} \tau \mid \tau\right)+\prod_{i=1}^{4} \theta_{1}\left(z_{i} \tau \mid \tau\right)}{2 \eta^{4}} .
\end{aligned}
$$

Open Access. This article is distributed under the terms of the Creative Commons Attribution License (CC-BY 4.0), which permits any use, distribution and reproduction in any medium, provided the original author(s) and source are credited.

\section{References}

[1] C. Angelantonj and A. Sagnotti, Open strings, Phys. Rept. 371 (2002) 1 [Erratum ibid. 376 (2003) 407] [hep-th/0204089] [INSPIRE].

[2] R. Blumenhagen, B. Körs, D. Lüst and S. Stieberger, Four-dimensional String Compactifications with D-branes, Orientifolds and Fluxes, Phys. Rept. 445 (2007) 1 [hep-th/0610327] [INSPIRE].

[3] R. Blumenhagen, M. Cvetič, P. Langacker and G. Shiu, Toward realistic intersecting D-brane models, Ann. Rev. Nucl. Part. Sci. 55 (2005) 71 [hep-th/0502005] [inSPIRE].

[4] L.E. Ibáñez and A.M. Uranga, String theory and particle physics: An introduction to string phenomenology, Cambridge University Press, Cambridge U.K. (2012).

[5] N. Arkani-Hamed and S. Dimopoulos, Supersymmetric unification without low energy supersymmetry and signatures for fine-tuning at the LHC, JHEP 06 (2005) 073 [hep-th/0405159] [INSPIRE].

[6] G.F. Giudice and A. Romanino, Split supersymmetry, Nucl. Phys. B 699 (2004) 65 [Erratum ibid. B 706 (2005) 487] [hep-ph/0406088] [INSPIRE].

[7] W. Buchmuller, M. Dierigl, F. Ruehle and J. Schweizer, Split symmetries, Phys. Lett. B 750 (2015) 615 [arXiv: 1507.06819] [InSPIRE].

[8] W. Buchmuller and J. Schweizer, Flavor mixings in flux compactifications, Phys. Rev. D 95 (2017) 075024 [arXiv: 1701.06935] [INSPIRE].

[9] C. Bachas, A Way to break supersymmetry, hep-th/9503030 [INSPIRE].

[10] A. Abouelsaood, C.G. Callan Jr., C.R. Nappi and S.A. Yost, Open Strings in Background Gauge Fields, Nucl. Phys. B 280 (1987) 599 [InSPIRE]. 
[11] R. Blumenhagen, L. Görlich, B. Körs and D. Lüst, Noncommutative compactifications of type-I strings on tori with magnetic background flux, JHEP 10 (2000) 006 [hep-th/0007024] [INSPIRE].

[12] C. Angelantonj, I. Antoniadis, E. Dudas and A. Sagnotti, Type I strings on magnetized orbifolds and brane transmutation, Phys. Lett. B 489 (2000) 223 [hep-th/0007090] [INSPIRE].

[13] M. Berkooz, M.R. Douglas and R.G. Leigh, Branes intersecting at angles, Nucl. Phys. B 480 (1996) 265 [hep-th/9606139] [INSPIRE].

[14] G. Aldazabal, S. Franco, L.E. Ibáñez, R. Rabadán and A.M. Uranga, D = 4 chiral string compactifications from intersecting branes, J. Math. Phys. 42 (2001) 3103 [hep-th/0011073] [INSPIRE].

[15] G. Aldazabal, S. Franco, L.E. Ibáñez, R. Rabadán and A.M. Uranga, Intersecting brane worlds, JHEP 02 (2001) 047 [hep-ph/0011132] [INSPIRE].

[16] L.E. Ibáñez, F. Marchesano and R. Rabadán, Getting just the standard model at intersecting branes, JHEP 11 (2001) 002 [hep-th/0105155] [INSPIRE].

[17] R. Blumenhagen, B. Körs, D. Lüst and T. Ott, The standard model from stable intersecting brane world orbifolds, Nucl. Phys. B 616 (2001) 3 [hep-th/0107138] [INSPIRE].

[18] M. Cvetič, G. Shiu and A.M. Uranga, Chiral four-dimensional $N=1$ supersymmetric type $2 A$ orientifolds from intersecting D6 branes, Nucl. Phys. B 615 (2001) 3 [hep-th/0107166] [INSPIRE].

[19] N.K. Nielsen and P. Olesen, An Unstable Yang-Mills Field Mode, Nucl. Phys. B 144 (1978) 376 [INSPIRE].

[20] I. Antoniadis and S. Dimopoulos, Splitting supersymmetry in string theory, Nucl. Phys. B 715 (2005) 120 [hep-th/0411032] [INSPIRE].

[21] I. Antoniadis, K. Benakli, A. Delgado, M. Quirós and M. Tuckmantel, Split extended supersymmetry from intersecting branes, Nucl. Phys. B 744 (2006) 156 [hep-th/0601003] [INSPIRE].

[22] W. Buchmuller, M. Dierigl, E. Dudas and J. Schweizer, Effective field theory for magnetic compactifications, JHEP 04 (2017) 052 [arXiv: 1611.03798] [INSPIRE].

[23] D.M. Ghilencea and H.M. Lee, Wilson lines and UV sensitivity in magnetic compactifications, JHEP 06 (2017) 039 [arXiv: 1703.10418] [INSPIRE]

[24] W. Buchmuller, M. Dierigl and E. Dudas, Flux compactifications and naturalness, JHEP 08 (2018) 151 [arXiv : 1804.07497] [INSPIRE].

[25] T. Hirose and N. Maru, Cancellation of One-loop Corrections to Scalar Masses in Yang-Mills Theory with Flux Compactification, JHEP 08 (2019) 054 [arXiv: 1904.06028] [INSPIRE].

[26] E. Dudas, G. Pradisi, M. Nicolosi and A. Sagnotti, On tadpoles and vacuum redefinitions in string theory, Nucl. Phys. B 708 (2005) 3 [hep-th/0410101] [InSPIRE].

[27] N. Marcus, A. Sagnotti and W. Siegel, Ten-dimensional Supersymmetric Yang-Mills Theory in Terms of Four-dimensional Superfields, Nucl. Phys. B 224 (1983) 159 [INSPIRE].

[28] N. Arkani-Hamed, T. Gregoire and J.G. Wacker, Higher dimensional supersymmetry in $4 D$ superspace, JHEP 03 (2002) 055 [hep-th/0101233] [INSPIRE]. 
[29] D. Cremades, L.E. Ibáñez and F. Marchesano, Computing Yukawa couplings from magnetized extra dimensions, JHEP 05 (2004) 079 [hep-th/0404229] [INSPIRE].

[30] J. Alfaro, A. Broncano, M.B. Gavela, S. Rigolin and M. Salvatori, Phenomenology of symmetry breaking from extra dimensions, JHEP 01 (2007) 005 [hep-ph/0606070] [INSPIRE].

[31] Y. Hamada and T. Kobayashi, Massive Modes in Magnetized Brane Models, Prog. Theor. Phys. 128 (2012) 903 [arXiv: 1207.6867] [INSPIRE].

[32] J. Wess and J. Bagger, Supersymmetry and supergravity, Princeton University Press, Princeton New Jersey U.S.A. (1992) [INSPIRE].

[33] J. Polchinski, String theory. Volume 2: Superstring theory and beyond, Cambridge University Press, Cambridge U.K. (1998) [INSPIRE].

[34] A. Sen, Tachyon dynamics in open string theory, Int. J. Mod. Phys. A 20 (2005) 5513 [hep-th/0410103] [INSPIRE].

[35] K. Hashimoto and S. Nagaoka, Recombination of intersecting D-branes by local tachyon condensation, JHEP 06 (2003) 034 [hep-th/0303204] [INSPIRE].

[36] F.T.J. Epple and D. Lüst, Tachyon condensation for intersecting branes at small and large angles, Fortsch. Phys. 52 (2004) 367 [hep-th/0311182] [INSPIRE].

[37] J. Bagger and A. Galperin, A New Goldstone multiplet for partially broken supersymmetry, Phys. Rev. D 55 (1997) 1091 [hep-th/9608177] [INSPIRE].

[38] M. Roček and A.A. Tseytlin, Partial breaking of global D $=4$ supersymmetry, constrained superfields and three-brane actions, Phys. Rev. D 59 (1999) 106001 [hep-th/9811232] [INSPIRE].

[39] A.A. Tseytlin, Born-Infeld action, supersymmetry and string theory, in The Many Faces of the Superworld, M.A. Shifman ed., World Scientific (2000), pp. 417-452 [hep-th/9908105] [INSPIRE].

[40] C. de Lacroix, H. Erbin, S.P. Kashyap, A. Sen and M. Verma, Closed Superstring Field Theory and its Applications, Int. J. Mod. Phys. A 32 (2017) 1730021 [arXiv:1703.06410] [INSPIRE].

[41] H. Georgi, Lie Algebras In Particle Physics. From Isospin To Unified Theories, Front. Phys. 54 (1982) 1 [INSPIRE]. 